hep-th/9907225, PUPT-1876, WIS-99/26/07-DPP

\title{
Dynamical Supersymmetry Breaking
}

\author{
Yael Shadmi \\ Department of Particle Physics \\ Weizmann Institute of Science, Rehovot 76100, Israel \\ and \\ Physics Department \\ Princeton University, Princeton, NJ 08544, USA \\ Yuri Shirman \\ Physics Department \\ Princeton University, Princeton, NJ 08544, USA
}

\begin{abstract}
Supersymmetry is one of the most plausible and theoretically motivated frameworks for extending the Standard Model. However, any supersymmetry in Nature must be a broken symmetry. Dynamical supersymmetry breaking (DSB) is an attractive idea for incorporating supersymmetry into a successful description of Nature. The study of DSB has recently enjoyed dramatic progress, fueled by advances in our understanding of the dynamics of supersymmetric field theories. These advances have allowed for direct analysis of DSB in strongly coupled theories, and for the discovery of new DSB theories, some of which contradict early criteria for DSB. We review these criteria, emphasizing recently discovered exceptions. We also describe, through many examples, various techniques for directly establishing DSB by studying the infrared theory, including both older techniques in regions of weak coupling,
\end{abstract}


and new techniques in regions of strong coupling. Finally, we present a list of representative DSB models, their main properties, and the relations between them.

Submitted to Reviews of Modern Physics 


\section{Contents}

I Introduction $\quad 5$

II Generalities $\quad 12$

A Vacuum Energy - The Order Parameter of SUSY Breaking, and $F$ and $D$ flatness . . . . . . . . . . . . . . . . . . . . . 12

B The Goldstind . . . . . . . . . . . . . . . . . . . . . 17

C Tree-Level Breaking . . . . . . . . . . . . . . . . . . 19

1 O'Raifeartaigh Models . . . . . . . . . . . . . . . . . . . . 19

2 Fayet-lliopoulos Breaking . . . . . . . . . . . . . . . . 22

III Indirect Criteria for DSB $\quad 24$

A The Witten Index . . . . . . . . . . . . . . . . . . . . . . . . . 24

B Global Symmetries and Supersymmetry Breaking . . . . . . . . . . . . . . 28

C Gaugino Condensation . . . . . . . . . . . . . . . . . 33

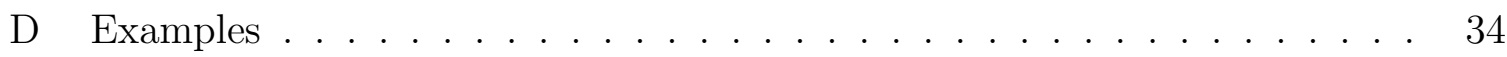

1 Spontaneously Broken Global Symmetry: the $S U(5)$ Model . . . . . . 34

2 Gaugino Condensation: the $S U(5)$ Model . . . . . . . . . . . . . . . . 35

3 R-symmetry and SUSY breaking . . . . . . . . . . . . . . . . . 35

4 Generalizations of the $S U(5)$ Model . . . . . . . . . . . . . . . . . . 37

IV Direct Analysis: Calculable Models $\quad 38$

A The 3-2 Model . . . . . . . . . . . . . . . . . . . . . . . . 39

B The 4-1 Model . . . . . . . . . . . . . . . . . . . . . . . . . . . . . . . . . 46

\begin{tabular}{|lll}
\hline Direct Analysis: Strongly-Coupled Theories & 47
\end{tabular}

A Supersymmetry Breaking Through Confinement . . . . . . . . . . . . . . 47

B Establishing Supersymmetry Breaking through a Dual Theory . . . . . . . 51

C Integrating Matter In and Out . . . . . . . . . . . . . . 57 
\begin{tabular}{|lll}
\hline VI & Violations of Indirect Criteria for DSB & 60
\end{tabular}

A Non-chiral Models . . . . . . . . . . . . . . . . . . . . . . 60

$1 \quad$ SUSY QCD with Singlets . . . . . . . . . . . . . . . . . . . . . 61

2 The Intriligator-Thomas-lzawa-Yanagida Model . . . . . . . . . . . . . 64

B Quantum Removal of Flat Directions. . . . . . . . . . . . . . . . . 69

C Supersymmetry Breaking with No R-symmetry . . . . . . . . . . . . . 74

\begin{tabular}{|lll}
\hline VII & DSB Models and Model Building Tools & 76
\end{tabular}

A Discarded Generator Models . . . . . . . . . . . . . . . . . . . . 77

B Supersymmetry Breaking from an Anomalous U(1) . . . . . . . . . . . . . 80

C List of Models and Literature Guide . . . . . . . . . . . . . . . . . . 83

\begin{tabular}{lr}
\hline APPENDIXES & 91
\end{tabular}

\begin{tabular}{|lll}
\hline A & Some Results on SUSY Gauge Theories & 91
\end{tabular}

1 Notations and Superspace Lagrangian . . . . . . . . . . . . . . . . . . . . 91

$2 \quad$ D-flat Directions $\ldots \ldots \ldots \ldots$

3 Pure Supersymmetric $S U\left(N_{c}\right)$ Theory $\ldots \ldots \ldots$

$4 \quad N_{f}<N_{c}:$ Affleck-Dine-Seiberg Superpotential . . . . . . . . . . . . . . 96

$5 \quad N_{f}=N_{c}:$ Quantum Moduli Space $\ldots \ldots \ldots . \ldots . \ldots 9$

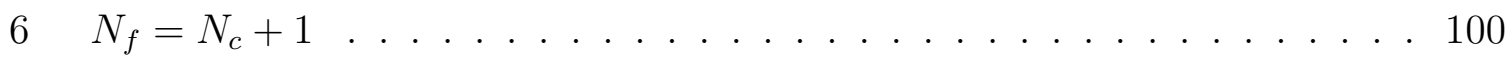

$7 \quad N_{f}>N_{c}+1:$ Dual Descriptions of the Infrared Physics $\ldots \ldots \ldots 1$

$8 \quad$ Other Models . . . . . . . . . . . . . . . . . . . . . . . . . . 103 


\section{INTRODUCTION}

Supersymmetry (SUSY), which rotates bosons into fermions and vice versa, is a beautiful theoretical idea. But nature is certainly not supersymmetric. If it were, we would see a fermionic partner for each known gauge boson, and a scalar partner for each known fermion, with degenerate masses. But experimentalists have been looking for "superpartners" long and hard, and so far in vain, pushing the limits on superpartner masses to roughly above a $100 \mathrm{GeV}$. Thus, any discussion of supersymmetry in nature is necessarily a discussion of broken supersymmetry.

Still, even broken supersymmetry is theoretically more appealing than no supersymmetry at all. First, supersymmetry provides a solution to the gauge hierarchy problem. Without supersymmetry, the scalar Higgs mass is quadratically divergent, so that the natural scale for it is the fundamental scale of the theory, e.g., the Planck scale, many orders of magnitude above the electroweak scale. In a supersymmetric theory, the mass of the scalar Higgs is tied to the mass of its fermionic superpartner. Since fermion masses are protected by chiral symmetries, the Higgs mass can naturally be around the electroweak scale, and radiative corrections do not destabilize this hierarchy. This success is not spoiled even when explicit supersymmetry-breaking terms are added to the Lagrangian of the theory, as long as these terms are "soft", that is, they only introduce logarithmic divergences, but no quadratic divergences, into scalar masses. The appearance of explicit supersymmetry-breaking terms in the low-energy effective theory can be theoretically justified if the underlying theory is supersymmetric, yet the vacuum state breaks supersymmetry spontaneously.

While spontaneously broken supersymmetry would explain the stability of the gauge hierarchy against radiative corrections, it still does not explain the origin of the hierarchy, that is, the origin of the small mass ratios in the theory. Indeed if supersymmetry were broken at the classical level (tree level), the scale of the soft terms would be determined by explicit mass parameters in the supersymmetric Lagrangian, and one would still have to understand why such parameters are so much smaller than the Planck scale. However, the origin of 
the hierarchy can be understood if supersymmetry is broken dynamically (Witten, 1981a). By "dynamical supersymmetry breaking" (DSB) we mean that supersymmetry is broken spontaneously in a theory that possesses supersymmetric vacua at the tree level, with the breaking triggered by dynamical effects. The crucial point about DSB is that if supersymmetry is unbroken at tree-level, supersymmetric non-renormalization theorems (Wess and Zumino, 1974; Ferrara-Iliopoulos-Zumind, 1974; Grisaru-Rocek-Siegel, 1979) imply that it remains unbroken to all orders in perturbation theory, and can therefore only be broken by non-perturbative effects, which are suppressed by roughly $e^{-8 \pi^{2} / g^{2}}$, where $g$ is the coupling. The electroweak scale is related to the size of the soft supersymmetry breaking terms, and thus it is proportional to the supersymmetry breaking scale. The latter is suppressed by the exponential above, and can easily be of the correct size, about 17 orders of magnitude below the Planck scale.

In addition, supersymmetry, or more precisely, local supersymmetry, provides the only known framework for a consistent description of gravity, in the context of string theory. If indeed the underlying fundamental physics is described by string theory, one can contemplate two qualitatively different scenarios. One is that SUSY is directly broken by stringy effects. Then, however, the SUSY breaking scale is generically around the string scale (barring new and better understanding of string vacua), and thus the gauge hierarchy problem is not solved by supersymmetry. Therefore, we shall focus here on a second possible scenario, namely, that in the low-energy limit, string theory gives rise to an effective field theory, and supersymmetry is spontaneously broken by the dynamics of this low-energy effective theory.

The aim of this review is then to describe the phenomenon of dynamical supersymmetry breaking in field theories with $\mathcal{N}=1$ global supersymmetry. ( $\mathcal{N}$ counts the number of supersymmetries. For $\mathcal{N}=1$ there are four supersymmetry charges and this is the smallest amount of supersymmetry allowed in four dimensions.)

The restriction on $\mathcal{N}$ comes from the fact that only $\mathcal{N}=1$ supersymmetry has chiral matter, which we need in the low-energy theory if it is to contain the standard model. Moreover, theories with $\mathcal{N}>1$ supersymmetry are believed to have an exact moduli space 
and thus are not expected to exhibit dynamical supersymmetry breaking.

The restriction to global supersymmetry still allows us to answer most of the questions we would be interested in. This situation is quite analogous to studying the breaking of a gauged bosonic symmetry in say, a theory with scalar matter. In that case one can determine the pattern of symmetry breaking just by studying the scalar potential. Similarly, we shall be able to determine whether supersymmetry is broken, and, if the theory is weakly coupled, what the vacuum energy and the light spectrum are. From our perspective, the most relevant consequence of "gauging" supersymmetry is the analogue of the Higgs mechanism by which the massless fermion accompanying supersymmetry breaking, the Goldstino, is eaten by the gravitino.

As we shall see, supersymmetry is broken if and only if the vacuum energy is non-zero. Furthermore, as we mentioned above, if supersymmetry is unbroken at tree level, it can only be broken by non-perturbative effects. Thus studying supersymmetry breaking requires understanding the non-perturbative dynamics of gauge theories in the infrared. Fortunately, in recent years, there has been tremendous progress in understanding the dynamics of supersymmetric field theories.

The potential of a supersymmetric theory is determined by two quantities, the Kähler potential, which contains the kinetic terms for the matter fields, and the superpotential, a holomorphic function of the matter fields which controls their Yukawa interactions. Holomorphy, together with the symmetries of the theory, may be used to determine the physical degrees of freedom and the superpotential of the infrared theory (Seiberg, 1994; Seiberg, 1995). Since the latter two are precisely the ingredients needed for studying supersymmetry breaking, this progress has fueled the discovery of many new supersymmetry breaking theories, as well as new techniques for establishing supersymmetry breaking.

The structure of this article is as follows. We start by describing general properties of

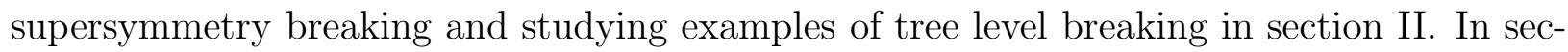
tion [II], we discuss indirect methods for finding theories with dynamical supersymmetry breaking, and for establishing supersymmetry breaking. As we shall see, these methods 
direct the search for supersymmetry breaking towards chiral theories with no flat directions, preferably possessing an anomaly free R-symmetry. These criteria do not amount to necessary conditions for supersymmetry breaking, and we shall point out "loopholes" in the indirect methods which allow the possibility of supersymmetry breaking in theories which violate all of the above requirements. Recent developments have led to the discovery of such SUSY breaking theories and we shall postpone the discussion of representative examples to section V1. Still, some of the indirect methods we shall describe, most notably, the breaking of a global symmetry in a theory with no flat directions, provide the most convincing evidence for supersymmetry breaking in theories that cannot be directly analyzed.

In sections $[\mathrm{V}$ and $\mathrm{V}$ we turn to theories that can be directly analyzed in the infrared. In the early 80's, such studies were limited to a semi-classical analysis in regions of weak coupling, and we shall describe such analyses in section IV. The main development in recent years has been the better understanding of supersymmetric theories in regions of strong coupling, and we shall study supersymmetry breaking in such theories in section $\square$. In both cases, the analysis of supersymmetry breaking involves two ingredients. The first is identifying the correct degrees of freedom of the theory, in terms of which the Kähler potential is non-singular. In all the theories we shall study, in the interior of the moduli space, these are either the confined variables, or the variables of a dual theory. I Indeed, we shall see that duality (Seiberg, 1995) - the fact that different UV theories may lead to the same infrared physics - can be a useful tool for establishing supersymmetry breaking, as one can sometimes pick a more convenient theory in which to study whether supersymmetry is broken or not. A second, related ingredient is finding the exact superpotential in terms of the light, physical degrees of freedom.

Having established these two ingredients, at low energies one then typically has a theory

\footnotetext{
${ }^{1}$ At the boundary of moduli space, the microscopic degrees of freedom will often be more appropriate.
} 
of chiral superfields, with all gauge dynamics integrated out, with a known superpotential. The problem of establishing supersymmetry breaking is then reduced to solving a system of equations to check whether or not the superpotential can be extremized.

Although the results we shall use on the infrared degrees of freedom and the exact superpotential apply to supersymmetric theories, they may still be used to argue for supersymmetry breaking, since the theories we shall study in this way are obtained by perturbing a supersymmetric theory. For a sufficiently small perturbation, the scale of supersymmetry breaking can be made sufficiently small, so that we can work above this scale and still use known results on the infrared supersymmetric theory. If supersymmetry is indeed broken in the theory, the breaking should persist even as the perturbation is increased. Otherwise, the theory undergoes a phase transition as some coupling is varied, being supersymmetric in some region and non-supersymmetric in another. However, one does not expect supersymmetric theories to undergo phase transitions as couplings are varied (Seiberg and Witten, 1994a; Seiberg and Witten, 1994b; Intriligator and Seiberg, 1994).

Having learned various techniques for the analysis of DSB, we use these in section $\nabla \mathbb{T}$ to study a few examples of theories that break supersymmetry dynamically even though they violate some of the criteria described in section [1].

Perhaps the most disappointing aspect of the recent progress in our understanding of supersymmetry breaking is that it still has not yielded any organizing principle to the study and classification of supersymmetry-breaking theories. In section VII we shall describe one method for generating new supersymmetry-breaking theories from known theories. However, this is far from a full, systematic classification. Nor can we tell immediately, without detailed analysis, whether a specific theory breaks supersymmetry or not. For these reasons we find it useful to present a rough survey of known models in section VII, pointing out their main features, the relations between them, and where applicable, their relevant properties for model-building purposes. While we shall see many different mechanisms by which supersymmetry is broken in these examples, the breaking is almost always the consequence of the interplay between instanton effects and a tree-level superpotential. 
Another area requiring further study is the analysis of supersymmetry-breaking vacua, their symmetries and light spectra, in strongly coupled theories. One may hope that recently discovered realizations of supersymmetric gauge theories as extended brane configurations in string theory will lead to further progress in this direction, as well as to some organizing principle for DSB. Indeed, several DSB models have been realized as D-brane configurations in string/M-theory, see for example (de Boer et at, 1998; Lykken-Poppitz-Trivedi, 1999). Moreover, in some cases the dynamical effects leading to supersymmetry breaking were understood in stringy language (de Boer et at, 1998). However, this approach has yet to lead to results which cannot be directly obtained in a field theory analysis.

We limit ourselves in this review to the theoretical analysis of supersymmetry breaking in different models. We do not discuss the questions of whether, and how, this breaking can feed down to the standard model. Ideally, a simple extension of the standard model would break supersymmetry by itself, generating an acceptable superpartner spectrum. Unfortunately, this is not the case. In simple supersymmetry breaking extensions of the Standard Model without new gauge interactions, non-perturbative effects would probably be too small to generate soft terms of the correct size (Affleck-Dine-Seiberg, 1985). Moreover, unless some of the scalars obtained their masses either radiatively, or from non-renormalizable operators, some superpartners would be lighter than the lightest lepton or quark Dimopoulos and Georgi, 1981). Thus, supersymmetry must be broken by a new, strongly-interacting sector, and then communicated to the SM either by supergravity effects, in which case the soft terms are generated by higher-dimension operators or at the loop level, or by gauge-interactions, in which case the soft terms occur at the loop-level. These different possibilities introduce different requirements on the SUSY breaking sector. For example, gravity mediation often requires singlet fields which participate in the SUSY breaking. Simple models of gauge mediation require a large unbroken global symmetry at the minimum of the SUSY breaking theory, in which the standard model gauge group can be embedded. Several of the supersymmetry breaking models discovered recently have some of these desired properties, and thus allow for improved phenomenological models for the communication of supersymmetry 
breaking.

Another issue which is important for phenomenological applications of DSB that we shall not address is the cosmological constant problem. In globally supersymmetric theories, fermionic and bosonic contributions to the vacuum energy cancel each other and the cosmological constant vanishes. Upon supersymmetry breaking this is no longer true, and the cosmological constant is comparable to the scale of supersymmetry breaking. While in a framework of local supersymmetry a further cancellation is possible, significant fine tuning is required. Eventually, a microscopic understanding of such a fine tuning is needed in any successful phenomenological application of dynamical supersymmetry breaking.

\section{How to Use This Review}

In the body of this review we assume that the reader is familiar with the general properties of supersymmetric field theories and rely heavily on symmetries and a number of exact non-perturbative results obtained in recent years. The reader who is just beginning the study of supersymmetry should first consult the Appendix, where we briefly present basic facts about supersymmetry and relevant results to make our presentation self-contained. For a more complete introduction to SUSY see for example, Bagger and Wes: (1991) and Nilles (1984). Several excellent reviews of the recent progress in the study of strongly coupled SUSY gauge theories exist, see for example 【ntriligator and Seiberg (1996), Peskin (1997), and Shifman (1997).

In Appendix A 1 we introduce notations and basic formulae for the Lagrangians of supersymmetric theories. The knowledge of these results is necessary in every section of the review. In A2 we discuss a method for finding the D-flat directions of a SUSY gage theory (directions along which the gauge interaction terms in the scalar potential vanish), and the parametrization of D-flat directions in terms of gauge-invariant operators. These results are necessary for the study of supersymmetry breaking in non-abelian gauge theories which we discuss starting in Section IIID. In A3 through A7 we turn to SUSY QCD with different numbers of flavors. While the results we present are used directly in various places in Sec- 
tions [V] VII, the discussion in these Appendixes also illustrates techniques in the analysis of the dynamics of general SUSY gauge theories and are applicable to models with matter transforming in general representations of the gauge group. The discussion also provides simple examples of phenomena such as theories with no quantum moduli spaces, deformed quantum moduli spaces, confinement without chiral symmetry breaking, and duality. We shall encounter these phenomena in various theories throughout the review. References to analyses of the dynamics of theories other than $S U(N)$ are collected in $\mathrm{A} 8$.

We also note that the reader who is only interested in a general knowledge of DSB can

skip sections $\mathrm{VI}$ and VII. Section VIIC can be used independently of the rest of the article as a guide to DSB models.

Finally, we note that the interested reader can find several useful reviews of dynamical supersymmetry breaking which have appeared in the past couple of years. A short introduction to recent developments can be found in (Skiba, 1997; Nelson, 1998; Poppitz, 1998; Thomas, 1998). Most notably, the review by Poppitz and Trivedi (1998), although smaller in scope than the present review, emphasizes recent developments in the field. It also contains a discussion of supersymmetry breaking in quantum mechanical systems. Shifman and Vainshtein (1999) give an excellent introduction to instanton techniques and discuss their application for supersymmetry breaking. The review by Giudice and Rattazzi (1998), focuses on applications of DSB to building models of gauge mediated supersymmetry breaking.

\section{GENERALITIES}

\section{A. Vacuum Energy - The Order Parameter of SUSY Breaking, and $F$ and $D$ flatness}

A positive vacuum energy is a necessary and sufficient condition for spontaneous SUSY breaking. This follows from the fact that the Hamiltonian of the theory is related to the absolute square of the SUSY generators (see Appendix A 1) 


$$
H=\frac{1}{4}\left(\bar{Q}_{1} Q_{1}+Q_{1} \bar{Q}_{1}+\bar{Q}_{2} Q_{2}+Q_{2} \bar{Q}_{2}\right)
$$

The energy is then either positive or zero. Furthermore, a state that is annihilated by $Q_{\alpha}$ has zero energy, and conversely, a zero-energy state is annihilated by $Q_{\alpha}$. Thus, the vacuum energy serves as an order parameter for supersymmetry breaking.

Therefore the study of supersymmetry breaking requires the knowledge of the scalar potential of the theory. It is convenient to formulate the theory in $\mathcal{N}=1$ superspace, where space-time (bosonic) coordinates are supplemented by anti-commuting (fermionic) coordinates]. In this formulation fields of different spins related by supersymmetry are combined in the supersymmetry multiplets, superfields. Matter fields form chiral superfields, while gauge bosons and their spin 1/2 superpartners form (real) vector superfields. In the superspace formulation, physics, and in particular, the scalar potential, is determined by two functions of the superfields, the superpotential and the Kähler potential. The superpotential encodes Yukawa-type interactions in the theory, in particular it contributes to the scalar potential. The superpotential is an analytic function of the superfields. This fact together with symmetries and known (weakly coupled) limits often allows to determine the exact non-perturbative superpotential of the theory. The Kähler potential, on the other hand, is a real function of superfields, and can only be reliably calculated when a weakly couled description of the theory exists. From our perspective, the Kähler potential is important in two respects. First, it gives rise to gauge-interaction terms in the scalar potential. Second, it determines the kinetic terms of the matter fields and thus modifies scalar interactions arising from the superpotential. Assuming a canonical (quadratic in the fields) Kähler potential, the scalar potential is

$$
V=\sum_{a}\left(D^{a}\right)^{2}+\sum_{i} F_{i}^{2}
$$

where the sum runs over all gauge indices $a$ and all matter fields $\phi_{i}$. In (2), the D-terms and F-terms are auxiliary components of vector and chiral superfields respectively. D-terms and

\footnotetext{
${ }^{2}$ For more detail see Appendix A 1.
} 
F-terms are not dynamical and one should solve their equations of motion. In particular, F-terms are given by derivatives of the superpotential (for more details, and the analogous expressions for D-terms, see Appendix (A 1).)

$$
F_{i}=\frac{\partial W}{\partial \phi_{i}} .
$$

For supersymmetry to remain unbroken, there has to be some field configuration for which both the $F$-terms and the $D$-terms vanish. ? $^{3}$ In fact, generically, such configurations exist not only at isolated points but on a subspace of the field space. This subspace is often referred to as the moduli space of the theory.

Classically, one could set all superpotential couplings to zero. Then the moduli space of the theory is the set of "D-flat directions", along which the $D$-terms vanish. A particularly useful parameterization of $D$-flat directions, which we discuss in Appendix A 2, can be given in terms of the gauge invariant operators of the theory (Luty and Taylor, 1996). Even when small tree-level superpotential couplings are turned on, the vacua will lie near the $D$-flat directions. It is convenient, therefore, to analyze SUSY gauge theories in two stages. First find the $D$-flat directions, then analyze the $F$-terms along these directions. The latter have classical contributions from the tree-level superpotential, and may "lift" some, or all, of the $D$-flat directions. Since a classical superpotential is a polynomial in the fields, $F$-terms typically grow for large scalar vacuum expectation values (vev), and vanish at the origin.

\footnotetext{
${ }^{3}$ Here we implicitly assumed that the Kähler potential is a regular function of the fields and has no singularities. For example, if the derivatives of the Kähler potential vanish, the scalar potential can be non-zero even if all $F$ and $D$ terms vanish, see Eq. (A6).

${ }^{4}$ An important exception are superpotentials terms that are linear in the fields, which lead to potentials that are nonzero even at the origin. Such terms necessarily involve gauge-singlets, and require the introduction of some mass scale. However, linear term can be generated dynamically. We shall encounter examples of trilinear, or higher, superpotential terms that become linear after confinement.
} 
As mentioned in the Introduction, a key point in the study of SUSY breaking is the fact that, due to the supersymmetric non-renormalization theorems (Wess and $\mathrm{Zu}$ mind, 1974; Ferrara-lliopoulos-Zumin,, 1974; Grisaru-Rocek-Siegel, 1979; Seiberg, 1993), the moduli space remains unmodified in perturbation theory. If the classical potential vanishes for some choice of vevs, it remains exactly zero to all orders in perturbation theory. Thus, only non-perturbative effects may generate a non-zero potential, and lift the classical zeros. Indeed, non-perturbative effects can modify the moduli space; they can lift the moduli space completely; or, finally, the quantum moduli space may coincide with the classical one.

There are numerous possibilities then for the behavior of the theory. If a theory breaks supersymmetry, it has some ground state of positive energy at some point in field space (or it may, in principle, have several ground states at different points). Alternatively, the theory may remain supersymmetric, with either one ground state of zero energy at some point in field space, or a few ground states, at isolated points, or with a continuum of ground states, corresponding to completely flat directions that are not lifted either classically or non-perturbatively. It is also possible that the theory does not have a stable vacuum state. In such a case, while a supersymmetric vacuum does not exist, the energy can become arbitrarily small along some direction on the moduli space.f While such a theory can still be given a cosmological interpretation (Affleck-Dine-Seiberg, 1984a), we shall not consider

\footnotetext{
${ }^{5}$ In this latter case the ground states are non-degenerate even if they appear to have the same energy in a certain approximation. This is because the low energy physics is non-supersymmetric, and the vacuum energy receives quantum corrections (on top of the non-perturbative effects which led to the non-vanishing energy in the first place). Since different non-supersymmetric vacua are non-equivalent, these quantum corrections lift the degeneracy.

${ }^{6}$ We shall call such directions in the moduli space "runaway" directions, and study them carefully in Sections VIA VIB.
} 
it a SUSY breaking theory for our purposes.

Note that supersymmetric theories are very different, in this respect, from other theories. In a non-supersymmetric theory, multiple ground states are usually related by a symmetry, and are therefore physically equivalent. On the other hand, different ground states of a supersymmetric theory may describe completely different physics. For example, classically, one flat direction of an $S U(3)$ gauge theory with two flavors is (see Appendix A 4 for details)

$$
f=\bar{f}=\left(\begin{array}{ll}
v & 0 \\
0 & 0 \\
0 & 0
\end{array}\right),
$$

where $f$ and $\bar{f}$ are the scalar components of the $S U(3)$ fundamentals and antifundamentals respectively. For any given choice of $v$, the low-energy theory is an $S U(2)$ gauge theory, whose gauge coupling depends on $v$. Note also that the flat directions of SUSY theories may extend to infinity, unlike the customary compact flat directions of bosonic global symmetries. Furthermore, in the case of other global symmetries, the existence of a flat direction is usually associated with spontaneous breaking of the symmetry, with the massless Goldstone bosons corresponding to motions along the flat direction. This is not the case with SUSY. The reason, of course, is that the SUSY generators do not correspond to motions in field space. Theories with unbroken SUSY may have degenerate vacua precisely because SUSY is unbroken. And, as we shall see in the next section, theories with spontaneously broken

\footnotetext{
${ }^{7}$ We also note that the runaway moduli may be stabilized, and supersymmetry broken, due to Kähler potential effects when the theory is coupled to gravity (Dvali and Kakushadze, 1998). However, since the typical vevs in this case will be of Planck size, the vacuum will be determined by the details of the microscopic theory at $M_{P}$, and the dynamical supersymmetry breaking is not calculable in the low energy effective field theory.

${ }^{8}$ Here we consider the classical vacua of the theory. Quantum mechanically, the flat directions (3) are lifted, and the theory does not have a stable vacuum. Yet, in many models non-equivalent quantum vacua exist.
} 
SUSY have the analog of Goldstone particles even when they only have a single ground state.

\section{B. The Goldstino}

The breaking of any bosonic global symmetry is accompanied by the appearance of massless Goldstone bosons that couple linearly to the symmetry current. Similarly, a theory with broken supersymmetry contains a massless fermion, which is usually referred to as a "Goldstone fermion", or in short, "Goldstino", that couples linearly to the SUSY current (Salam and Strathdee, 1974; Witten, 1981a).

The Goldstino coupling to the SUSY current can be expressed as

$$
J_{\alpha}^{\mu}=f \sigma_{\alpha}^{\mu \dot{\beta}} \psi_{\dot{\beta}}^{G}+\ldots,
$$

where $\psi_{\dot{\beta}}^{G}$ is the Goldstino, and as we shall see momentarily, $f$ is a constant which is nonzero when SUSY is broken. The ellipsis in (4) stand for terms quadratic in the fields and for potential derivative terms. Conservation of the SUSY current then implies that the Goldstino is massless. To justify (4), note that, for broken SUSY (Witten, 1981a),

$$
\int d^{4} x \partial_{\eta}\left\langle 0\left|T J_{\alpha}^{\eta}(x) J_{\dot{\beta}}^{\nu}(0)\right| 0\right\rangle=\left\langle 0\left|\left\{Q_{\alpha}, J_{\dot{\beta}}^{\nu}(0)\right\}\right| 0\right\rangle \neq 0 .
$$

If indeed there is a massless fermion coupling to the current as in (4), then the LHS of (5) is equal to

$$
f^{2} \sigma_{\alpha}^{\eta \dot{\alpha}} \sigma_{\dot{\beta}}^{\nu \beta} \int d^{4} x \partial_{\eta}\left\langle 0\left|T \psi_{\dot{\alpha}}^{G}(x) \psi_{\beta}^{G}(0)\right| 0\right\rangle=f^{2} \sigma_{\alpha}^{\eta \dot{\alpha}} \sigma_{\dot{\beta}}^{\nu \beta}\left[-i p_{\eta} G(p)_{\dot{\alpha} \beta}\right]_{p \rightarrow 0}=f^{2} \sigma_{\alpha \dot{\beta}}^{\nu},
$$

where $G(p)_{\dot{\alpha} \dot{\beta}}$ is the Goldstino propagator. So indeed $f$ is nonzero. Note that a fermion with derivative coupling to the current would not contribute to the RHS of (6) because of the additional factors of the momentum in the numerator. In fact, since from the SUSY algebra $\left\langle 0\left|\left\{Q_{\alpha}, J_{\dot{\beta}}^{\nu}(0)\right\}\right| 0\right\rangle=2 E \sigma_{\alpha \dot{\beta}}^{\nu}$, with $E$ the energy, we have $f^{2}=2 E$.

To see the appearance of the Goldstino more concretely, consider the SUSY current 


$$
J_{\alpha}^{\mu} \sim \sum_{\phi} \frac{\delta \mathcal{L}}{\delta\left(\partial_{\mu} \phi\right)}(\delta \phi)_{\alpha},
$$

where the sum is over all fields, and $(\delta \phi)_{\alpha}$ is the shift of the field $\phi$ under a SUSY transformation. Because of Lorentz symmetry, the only linear terms in (7) come from vacuum expectation values of $(\delta \phi)$. Examining the SUSY transformations of the chiral and vector multiplets of $\mathcal{N}=1$ SUSY (see for example Bagger and Wess, (1991)), we see from Lorentz invariance that the only fields whose SUSY transformations contain Lorentz-invariant objects, which can develop vevs, are the matter fermion $\psi_{i}$, whose SUSY transformation gives $F_{i}$, and the gauge fermion $\lambda^{a}$, whose transformation gives $D^{a}$. One then finds,

$$
\psi^{G} \sim \sum\left\langle F_{i}\right\rangle \psi_{i}+\frac{1}{\sqrt{2}} \sum\left\langle D^{a}\right\rangle \lambda^{a},
$$

so that the Goldstino is a linear combination of the chiral and gauge fermions whose auxiliary fields $F$ and $D$ acquire vevs. Note that Eq. (8) actually only holds with a canonical (quadratic) Kähler potential, otherwise derivatives of the Kähler potential enter as well. We can use this to argue that a non-vanishing $F$-vev or $D$-vev is a necessary condition for SUSY breaking. When SUSY is broken, there is a massless fermion, the Goldstino, that transforms inhomogeneously under the action of the SUSY generators. But the only Lorentz-invariant objects that appear in the SUSY variations of the $\mathcal{N}=1$ multiplets, and therefore may obtain vevs, are the auxiliary fields $F$ and $D$. Thus, for SUSY breaking to occur, some $F$ or $D$ - fields should develop vevs.

In light of the above, it would first seem that if SUSY is relevant to nature, we should observe the massless Goldstino. However, we ultimately need to promote SUSY to a local symmetry to incorporate gravity into the full theory. In the framework of local supersymmetry, the massless Goldstino becomes the longitudinal component of the Gravitino, much like in the case of the Higgs mechanism. The Gravitino then has a coupling to ordinary matter other than the gravitational interaction, by virtue of its Goldstino component. It should come as no surprise then, that the Gravitino mass as well as its coupling to matter fields, are related to the SUSY breaking scale. This has important phenomenological implications. In particular, in models with low-scale SUSY breaking, the gravitino is very light, 
and the decay of other superpartners into the gravitino may be observed in collider searches for supersymmetry (Dimopoulos et a4, 1998; Stump-Wiest-Yuan, 1996).

\section{Tree-Level Breaking}

\section{O'Raifeartaigh Models}

One of the simplest models of spontaneous supersymmetry breaking was proposed by O'Raifeartaigh (1975), and is based on a theory of chiral superfields. Supersymmetry in the model is broken at tree level: while the lagrangian of the model is supersymmetric, even the classical potential is such that a supersymmetric vacuum state does not exist.

In addition to giving the simplest example of spontaneously broken supersymmetry, the study of O'Raifeartaigh models will be useful for our later studies of DSB, as the low-energy description of many dynamical models we shall encounter will be given by an O'Raifeartaightype model.

Before writing down the simplest example of an O'Raifeartaigh model, let us describe the general properties of such models. First, we shall restrict our attention to superpotentials with only positive exponents of the fields. We shall later analyze a number of models where the low-energy description involves superpotentials containing negative exponents of the fields. Such terms, however, are generated by the non-perturbative dynamics of the underlying (strongly coupled) microscopic theory, and are not appropriate in the tree level superpotential we consider here.

Second, since the superpotential is a polynomial in the fields, at least one of the fields in this model needs to appear linearly in the superpotential, or there will be a supersymmetric vacuum at the origin of the moduli space.

It would prove useful, for future purposes, to pay special attention to the R-symmetry of the model. (For the definition of an R-symmetry, see Appendix (A1)). If this symmetry is unbroken by the superpotential, there is then at least one field of R-charge 2. More 
generally, consider a model containing fields $\phi_{i}^{c}, i=1, \ldots, k$ with $\mathrm{R}$-charge 2 , and fields $\phi_{a}^{n}, a=1, \ldots, l$ with R-charge 0 . (For convenience, we shall call them charged and neutral respectively, even though various components of the superfields transform differently under R-symmetry.) The most general superpotential respecting the R-symmetry can be written as

$$
W=\sum_{i=1}^{k} \phi_{i}^{c} f_{i}\left(\phi_{a}^{n}\right)
$$

and, for supersymmetry to break, at least one of the $f_{i}$ 's, say $f_{1}$, contains a constant term, independent of the fields. The equations of motion for the R-charged fields $\partial W / \partial \phi_{i}^{c}=$ $f_{i}\left(\phi_{a}^{n}\right)=0$ give $k$ equations for $l$ unknowns $\phi_{a}^{n}$. If $k>l$ there are no solutions for generic functions $f_{i}$, the F-term conditions can not all be satisfied and supersymmetry is broken.

One can modify these models by adding fields with R-charges $0<Q_{R}<2$. Since such fields can not couple to the fields $\phi_{i}^{c}$ while preserving the R-symmetry, they will not change the above discussion, and supersymmetry remains broken. If, on the other hand, fields with negative R-charges are added to the model, the total number of variables on which the $f_{i}$ 's depend increases, and in general supersymmetry is unbroken. Finally, we should note that adding to the superpotential explicit $R$ symmetry violating couplings which do not involve fields of $\mathrm{R}$ charge 2 will not modify the above discussion. On the other hand, $\mathrm{R}$ symmetry violating terms which include fields of $\mathrm{R}$ charge 2 will generically lead to the restoration of supersymmetry.

It is also useful to look at the equations of motion for the R-neutral fields. First, note that their vev's are fixed by minimizing the part of the scalar potential arising from the F-terms of the R-charged fields (the remaining terms in the scalar potential vanish at least when $\left\langle\phi_{i}^{c}\right\rangle=0$ for all $i$ ). Therefore, there are $l$ F-term equations depending on $k$ independent variables $\phi_{i}^{c}$. In a SUSY breaking model $k>l$, so there are $k-l$ linear combinations of the fields $\phi_{i}^{c}$ that are left undetermined. Thus O'Raifeartaigh models necessarily posses directions of flat (non-zero) potential in the tree level approximation (Zuming, 1981; Einhorn and Jones, 1983; Polchinski, 1983). As we shall discuss later this is not a generic situation 
in models of DSB.

The simplest example of an O'Raifeartaigh model requires two fields with R-charge 2, one field with $\mathrm{R}$-charge $0(k=2, l=1)$, and has the superpotential

$$
W=\phi_{1}\left(M_{1}^{2}-\lambda_{1} \phi^{2}\right)+m_{2} \phi \phi_{2}
$$

It is easy to see that the F-term conditions for $\phi_{1}$ and $\phi_{2}$ are incompatible. We can directly minimize the scalar potential

$$
V=\left|M_{1}^{2}-\lambda_{1} \phi^{2}\right|^{2}+\left|m_{2} \phi\right|^{2}+\left|m_{2} \phi_{2}-2 \lambda_{1} \phi_{1} \phi\right|^{2}
$$

The first two terms in (11) determine the value of $\phi$ at the minimum. In the limit $m_{2}^{2} /\left(\lambda_{1} M_{1}^{2}\right) \gg 1$ the minimum is found at $\phi=0$, while for small $m_{2}^{2} /\left(\lambda_{1} M_{1}^{2}\right)$ we find $\phi=\left(M_{1}^{2}-m_{2}^{2} / 2\right)^{1 / 2} / \lambda_{1}$. Note that $\phi_{1}$ and $\phi_{2}$ only appear in the last term in (11). This term should be set to zero for the potential to be extremal with respect to $\phi_{1}$ and $\phi_{2}$. This is achieved when $\phi_{2}=-2 \lambda_{1} \phi \phi_{1} / m_{2}$, and therefore, at tree level the linear combination $m_{2} \phi_{1}-2 \lambda_{1}\langle\phi\rangle \phi_{2}$ is arbitrary, as was expected from the previous discussion. Equivalently, we can parameterize different vacua by the expectation values of $\phi_{1}$. Note that different vacua are physically non-equivalent, in particular the spectrum depends on $\left\langle\phi_{1}\right\rangle$.

It is easy to find the tree level spectrum of the model. For any choice of parameters it contains a massless fermion, the Goldstino. The spectrum also contains the scalar field associated with the flat direction whose mass arises entirely due to radiative corrections (however, there are no quadratic divergences since the action of the theory is supersymmetric although supersymmetry is not realized linearly). All other states are massive. Another important feature of this spectrum is that the supertrace of the mass matrix squared, $\mathrm{STr} m_{i}^{2}$, vanishes. This property of the spectrum holds for any model with tree level supersymmetry breaking (Ferrara-Girardello-Palumbo, 1979).

Since supersymmetry is broken, the vacuum degeneracy is lifted in perturbation theory. Hud (1976) calculated the one loop corrections to the potential of this model. They are given by 


$$
\Delta V\left(\phi_{1}\right)=\sum_{i} \frac{(-1)^{F}}{64 \pi^{2}} m_{i}\left(\phi_{1}\right)^{4} \ln \left(\frac{m_{i}\left(\phi_{1}\right)^{2}}{\mu^{2}}\right),
$$

where the sum is over all massive fields (and the masses depend on the $\phi_{1}$ vev). Hud (1976) found that the corrections generate a positive mass for $\phi_{1}$, and that the non-supersymmetric vacuum is located at $\phi_{1}=0$ with unbroken R-symmetry. He also analyzed a model with an $S U(3)$ global symmetry and a model constructed by Fayet (1975) with $S U(2) \times U(1)$ symmetry, and in both cases found that the tree-level modulus acquires positive mass due to one-loop corrections to the Kähler potential, leading to the unique vacuum with unbroken Rsymmetry. In fact, this conclusion is not surprising. In the model discussed above, quantum corrections to the vacuum energy come from the renormalization of the mass parameter $M^{2}$. Due to the holomorphy of the superpotential, these are completely determined by the wave function renormalization of $\phi_{1}$ and, since the model is infrared free, necessarily generate

positive contribution to the scalar potential. It is important to note that in modifications of the model that include gauge fields, there may be a negative contribution to the potential. The balance of the two perturbative effects may produce a stable minimum at large values of the modulus vev (Witten, 1981b).

\section{Fayet-Iliopoulos Breaking}

Another useful example of tree level supersymmetry breaking is given by a model with $U(1)$ gauge interactions (Fayet-lliopoulos, 1974). In this model supersymmetry breaking is driven by D-term contributions to the potential, but depending on the parameters of the Lagrangian, the non-zero vacuum energy either comes entirely from D-term contributions, or from both D- and F-terms. To understand how D-terms can drive supersymmetry breaking, we recall that the Kähler potential can be written as a function of the gauge invariant combination of fields

$$
K=f\left(\phi^{\dagger} e^{V} \phi, \mathcal{W}^{\dagger} \mathcal{W}, \mathcal{S}\right)
$$


where $\phi$ represents matter fields transforming in some representation of the gauge group, $V$ is a vector superfield whose supersymmetric field strength is $\mathcal{W}$, and $S$ represents gauge singlet fields. In a nonabelian theory this is the only possible form of field dependence in the Kähler potential. In an abelian theory, however, the D-term of the vector superfield $V$ is invariant under the gauge and supersymmetry transformations by itself. Thus, if one does not require parity invariance, the lowest order Kähler potential of a $U(1)$ gauge theory can be written as s $^{9}$

$$
K=Q^{\dagger} e^{V} Q+\bar{Q}^{\dagger} e^{-V} \bar{Q}+\xi_{F I} V
$$

This Kähler potential together with superpotential mass terms for the matter fields leads to the following scalar potential

$$
V=\frac{g^{2}}{2}\left(|Q|^{2}-|\bar{Q}|^{2}+\xi\right)^{2}+m^{2}\left(|Q|^{2}+|\bar{Q}|^{2}\right)
$$

It is easy to see that the vacuum energy determined by this potential is necessarily positive and supersymmetry is broken. When $g^{2} \xi<m^{2}$ both scalar fields have positive mass and their vevs vanish. The positive contribution to the vacuum energy comes entirely from the D-term in the potential. The scalar mass matrix has eigenvalues $m_{ \pm}^{2}=m^{2} \pm g^{2} \xi$. The gauge symmetry is unbroken, and thus the gauge boson remains massless. The matter fermions retain their mass $m$, while the gaugino remains massless and plays the role of the Goldstino. (In accord with with the fact that here $\left\langle F_{i}\right\rangle=0$ and $\langle D\rangle \neq 0$, see Eq. 8.)

When $g^{2} \xi>m^{2}$, the field $\bar{Q}$ has negative mass and acquires a vev. At the minimum of the potential $Q=0$ and $\bar{Q}=v$, where $v=\left(2 \xi-4 m^{2} / g^{2}\right)^{1 / 2}$. We see that both the gauge symmetry and supersymmetry are broken. Moreover, both D-term and F-term are non-vanishing and supersymmetry breaking is of the mixed type. One can easily find that the spectrum of the model contains one vector field and one real scalar field of mass squared $\frac{1}{2} g^{2} v^{2}$, one complex scalar of mass squared $2 m^{2}$, two fermions of mass $\left(m^{2}+\frac{1}{2} g^{2} v^{2}\right)^{1 / 2}$, and a

\footnotetext{
${ }^{9}$ We restrict our attention to two matter multiplets with charges \pm 1 .
} 
massless Goldstone fermion which is a linear combination of the Goldstino and the positively charged fermion

$$
\tilde{\lambda}=\frac{1}{\sqrt{m^{2}+\frac{1}{2} g^{2} v^{2}}}\left(m \lambda+\frac{i g v}{\sqrt{2}} \psi_{Q}\right) .
$$

\section{INDIRECT CRITERIA FOR DSB}

As we have seen in Section ПIA, the fact that the vacuum energy is the relevant order parameter immediately points the way in our quest for SUSY breaking: we should study the zeros of the scalar potential. This, indeed, is what we shall undertake to do in sections IV and onward. Unfortunately, directly studying the zeros of the potential will not always be possible, or easy. In this Section we review several alternate "indirect" methods that are useful in the search for supersymmetry breaking.

\section{A. The Witten Index}

Supersymmetry breaking is related to the existence of zero-energy states. Rather than looking at the total number of zero energy states, it is often useful to consider the Witten

index (Witten, 1982), which measures the difference between the number of bosonic and fermionic states of zero energy,

$$
\operatorname{Tr}(-1)^{F} \equiv n_{B}^{0}-n_{F}^{0}
$$

If the Witten index is nonzero, there is at least one state of zero energy, and supersymmetry is unbroken. If the index vanishes, supersymmetry may either be broken, with no states of zero energy, or it may be unbroken, with identical numbers of fermionic and bosonic states of zero energy.

The Witten index is a topological invariant of the theory. In this lies its usefulness. It may be calculated for some convenient choice of the parameters of the theory, and in particular, for weak coupling, but the result is valid generally. To see this, note that in a 
finite volume, fermionic and bosonic states of positive energy are paired by the action of the SUSY generator:

$$
Q\left|b_{E}\right\rangle \sim \sqrt{E}\left|f_{E}\right\rangle \quad Q\left|f_{E}\right\rangle \sim \sqrt{E}\left|b_{E}\right\rangle
$$

where $\left|b_{E}\right\rangle\left(\left|f_{E}\right\rangle\right)$ is a bosonic (fermionic) state of energy $E$. Recall that states of zero energy are annihilated by $Q$, and are therefore not paired). Thus, under "mild" variations of the parameters of the theory, states may move to zero energy and from zero energy, but they always do so in Bose-Fermi pairs, leaving the Witten index unchanged.

Let us be a bit more precise now about what is meant by "mild" variations above. As long as a parameter of the theory, which is originally nonzero, is varied to a different nonzero value, we do not expect the Witten index to change, since different states can only move between different energy levels in pairs. The danger lies in the appearance of new states of zero energy. This can happen if the asymptotic (in field space) behavior of the potential changes, which may happen if some parameter of the theory is set to zero, or is turned on. In that case, states may "come in" from infinity or "move out" to infinity.

The index of several theories was calculated by Witten (1982). In particular, Witten found that the index of a pure supersymmetric Yang-Mills (SYM) theory is non-zero. $\square$ Thus, these theories do not break supersymmetry spontaneously. An important corollary is

\footnotetext{
${ }^{10}$ This in fact justifies including only zero-energy states in (17). States of non-zero energy do not contribute.

${ }^{11}$ For $S U$ and $S P$ groups the index is equal to $r+1$, where $r$ is the rank of the group. This is the same as the number of gaugino condensates for these groups. More generally, the index equals the dual coxeter number of the group, which is different from $r+1$ for some groups, notably some of the $S O$ groups (Witten, 1998). The fact that the number of gaugino condensates does not always equal $r+1$, which was believed to be the value of the index, remained a puzzle until its resolution by Witten recently (Witten, 1998). This puzzle partly motivated the conjecture that SYM theories have a vacuum with no gaugino condensate (Kovner and Shifman, 1997). This possibility would
} 
that SYM theories with massive matter (and no massless matter) do not break supersymmetry either. The reason is that, at least in weak coupling, one can take all masses large, so that there are no massless states in these theories beyond those of the pure SYM theory, and so the value of the index is the same as in the pure SYM theory.

What happens when the mass of the matter fields is taken to zero? The theory with zero mass has flat directions, along which the potential is classically zero (away from these flat directions the potential behaves as the fourth power of the field strength). In contrast, the theory with mass for all matter fields has no classical flat directions, with the potential growing at least quadratically for large fields. Thus, as the mass is taken to zero, the asymptotic behavior of the potential changes, and the Witten index may change too. In fact, the index is ill-defined in the presence of flat directions, since zero modes associated with the flat directions lead to a continuous spectrum of states. (Indeed, to calculate the index of any theory one needs to consider the theory in a finite volume so that the resulting spectrum is discrete.) We therefore cannot say anything about supersymmetry breaking in massless, non-chiral theories based on the Witten index of the pure SYM theory.

Consider for example SQCD with $N$ colors and $F$ flavors of mass $m$, which we discuss in Appendix A4 A7. As explained there, in the presence of mass terms $m_{j}^{i} Q_{i} \cdot \bar{Q}^{j}$, the theory has $N$ vacua at

$$
M_{i}^{j} \equiv Q_{i} \cdot \bar{Q}^{j}=\Lambda^{(3 N-F) / N}(\operatorname{det} m)^{1 / N}\left(m^{-1}\right)_{i}^{j}
$$

corresponding to the $N$ roots of unity. This is in agreement with the Witten index $N$ of pure $S U(N)$ gauge theory. Consider now the massless limit, $m_{j}^{i} \rightarrow 0$. For $F<N$, the vacua (19) all tend to infinity. The theory has no ground state at finite field vevs. The potential is

have far-reaching consequences for supersymmetry breaking. The vast majority of theories that break SUSY do so by virtue of a superpotential generated by gaugino condensation. A vacuum with no gaugino condensate would mean an extra ground state, or an entire branch of ground states, with zero energy and unbroken SUSY. 
nonzero in any finite region of field space and slopes to zero at infinity. The massless limit of the theory is therefore not well-defined. For $F>N$, by taking $m_{j}^{i} \rightarrow 0$ in different ways, any value of $M_{i}^{j}$ may be attained. The massless theory has an entire moduli space of vacua, parameterized by $M_{i}^{j}$. The $N=F$ case is more subtle, but in this case too, the theory has a moduli space of vacua. Thus, the ground states of the massless $S U(N)$ theory with $F$ flavors are drastically different from those of the massive theory. In these examples we explicitly see how zero-energy states can disappear to infinity, or come in from infinity. Again, this is possible because the asymptotic behavior of the potential changes as the mass tends to zero. The theory including mass terms has no flat directions. Asymptotically the potential rises at least quadratically. The massless theory has classical flat directions. Quantum mechanically, they are completely lifted for $F<N$, and the potential asymptotes to zero as a fractional power of the field. For $F \geq N$ flat directions remain even quantum mechanically. In any case, adding mass terms changes the asymptotic behavior of the potential.

In the examples above, the massive theory was supersymmetric (with zero energy states at finite fields vevs) and the massless theory was either supersymmetric (with a continuum of vacua) or not well defined (with no ground state). It is natural to ask whether there exist vectorlike (parity-conserving) theories that break SUSY as the relevant masses are taken to zero. The answer to this question is affirmative as we shall see in an explicit example in Section VIA2. It is useful to understand the general properties of the potential in such a theory. As before, we expect the fully massive theory to have a non-zero Witten index. The only way to obtain supersymmetry breaking as the masses are taken to zero, is if the masses change the asymptotic behavior of the potential. Suppose that for any finite value of the mass parameter $m$, the theory possesses a supersymmetric vacuum at some vev $v_{0}(m)$, which moves away to infinity as some of the masses are taken to zero. Clearly, for small finite masses, the directional derivative of the potential with respect to the modulus is negative for large values of $v<v_{0}(m)$. (The theory may have various minima for finite values of $v$, but we are interested in the asymptotic behavior of the potential at large $v$.) In the absence of a phase transition at zero mass, such a directional derivative will remain 
non-positive in the limit $m \rightarrow 0$. However, there are still two possibilities. First, it is possible that the directional derivative is negative for any finite vev, and only vanishes in the double limit, $m \rightarrow 0, v \rightarrow \infty$. In such a case the theory does not have a stable vacuum. However, it is also possible that the derivative vanishes in the limit $m \rightarrow 0$ for sufficiently large, but otherwise arbitrary $v$. If this is the case, the asymptotic behavior of the potential changes, and it becomes a non-zero constant asymptotically far along the flat direction, so supersymmetry is broken. However, because the potential is flat, running effects cannot be neglected. Indeed, as we shall argue in Section VIA 2, such effects may lift the vacuum degeneracy and determine the true non-supersymmetric vacuum.

To summarize, pure SYM theories, as well as vectorlike theories with masses for all matter fields, have a non-zero index, and do not break supersymmetry. When some masses are taken to zero, the resulting theories have classical flat directions, and therefore, the asymptotic behavior of the potential is different from that of the massive theory. The Witten index may then change discontinuously and differ, if it is well defined, from that of the massive theory.

What about chiral (parity-violating) theories? In such theories, at least some of the matter fields cannot be given mass. Thus, these theories cannot be obtained by deforming a massive vectorlike theory, and there is no a priori reason to expect, based on existing computations of Witten index, that these theories are supersymmetric. Indeed, most known examples of supersymmetry breaking are chiral.

\section{B. Global Symmetries and Supersymmetry Breaking}

In this section we shall discuss the connection between global symmetries and supersymmetry breaking, which motivates two criteria for supersymmetry breaking. While these are useful guidelines for finding supersymmetry-breaking theories, they are not strict rules, and we shall encounter several exceptions in the following.

Consider first a theory with an exact, non-anomalous global symmetry, and no flat directions. If the global symmetry is spontaneously broken, there is a massless scalar field, 
the Goldstone boson, with no potential. With unbroken supersymmetry, the Goldstone boson is part of a chiral supermultiplet that contains an additional massless scalar, again with no potential. This scalar describes motions along a flat direction of zero potential. But this contradicts our initial assumption that there are no flat directions. To avoid the contradiction we should drop the assumption of unbroken supersymmetry. This gives a powerful tool for establishing supersymmetry breaking (Affleck-Dine-Seiberg, 1984b; AffleckDine-Seiberg, 1985): If a theory has a spontaneously broken global symmetry and no flat directions, the theory breaks supersymmetry.

We have assumed here that the additional massless scalar corresponds to motions along a non-compact flat direction. This is often the case, since, in supersymmetric theories, the superpotential is invariant under the complexified global symmetry, with the Goldstone boson corresponding to the imaginary part of the relevant order parameter, and its supersymmetric partner corresponding to the real part of the order parameter.

In general, deciding whether a global symmetry is broken requires detailed knowledge of the potential of the theory, and is at least as hard as determining whether the vacuum energy vanishes. However, if a theory is strongly coupled at the scale at which supersymmetry might be broken, one cannot directly answer either of these questions. ${ }^{30}$ Still, in some cases, one may argue, based on 't Hooft anomaly matching conditions (t Hooft, 1980), that a global symmetry is broken.

If a global symmetry is unbroken in the ground-state, then the massless fermions of

\footnotetext{
${ }^{12}$ The possibility that the low-energy theory is a theory of Goldstone bosons and massless chiral fields such that the supersymmetric scalar partner of any Goldstone is a Goldstone too is ruled out. Such theories can only be coupled to gravity for discrete values of Newton's constant BaggerWitten, 1982), and so cannot describe the low-energy behavior of renormalizable gauge theories.

${ }^{13}$ The most obvious example is a theory which does not possess any adjustable parameters, and has only one scale, like an $S U(5)$ model of Section IIID.
} 
the low-energy theory should reproduce the global triangle anomalies of the microscopic theory ( $\mathrm{t}$ Hooft, 1980). Thus there should be a set of fields, with appropriate charges under the global symmetry, that give a solution to the anomaly-matching conditions. This fact may be used when trying to determine whether a theory confines, and how its global symmetries are realized in the vacuum. For example, if the gauge-invariants which can be constructed out of the microscopic fields of the theory saturate the anomaly-matching conditions for some subgroup of the global symmetry of the microscopic theory, it is plausible that the theory confines, and that the relevant symmetry subgroup remains unbroken in the vacuum. In contrast, if all possible solutions to the anomaly-matching conditions are very complicated, that is, they require a large set of fields, it is plausible to conclude that the global symmetry is spontaneously broken.

In the case of an R-symmetry there is another way to determine whether it is spontaneously broken. In many theories, the scale of supersymmetry breaking is much lower than the strong-coupling scale, so that supersymmetry breaking can be studied in a lowenergy effective theory involving chiral superfields only, with all gauge dynamics integrated out. In fact, the low-energy theory is an O'Raifeartaigh-like model (with possibly negative exponents of the fields in the superpotential arising from non-perturbative effects in the microscopic description). In some cases it is easy to see that the origin is excluded from the moduli space, because, for example, the potential diverges there. Then typically, some terms appearing in the superpotential obtain vevs. Since all terms in the superpotential have R-charge 2 this implies that R-symmetry is broken. Obviously such an argument is not applicable to other global symmetries because the superpotential is necessarily neutral under non-R symmetries. So it is often easier to prove that an R-symmetry is broken, than to prove that a non-R symmetry is broken. If the theory has no flat directions one can then conclude that supersymmetry is broken.

It is not surprising that R-symmetries, which do not commute with supersymmetry, should play a special role in supersymmetry breaking. Let us discuss this role further, following Nelson and Seiberg (1994). 
In what follows we shall assume that the gauge dynamics was integrated out. Suppose we have a low-energy theory with a superpotential $W\left(\left\{X_{i}\right\}\right)$, where $X_{i}$ are chiral fields and $i=1 \ldots n$. For supersymmetry to remain unbroken, the superpotential should be extremal with respect to all fields,

$$
\frac{\partial W}{\partial X_{i}}=0
$$

If the theory has no symmetries, the number of unknowns, $X_{i}$, equals the number of equations. Similarly, if the theory has a global symmetry that commutes with supersymmetry, the number of equations equals the number of unknowns. To see this note that in this case, the superpotential can only depend on chiral field combinations that are invariant under the symmetry. Therefore, if there are $k$ symmetry generators, the superpotential depends on $n-k$ invariant quantities (for example, for a $U(1)$ symmetry these could be $X_{i} / X_{1}^{\frac{q_{i}}{q_{1}}}$, where $i=2 \ldots n$, and $q_{i}, q_{1}$ are the $U(1)$ charges of $X_{i}, X_{1}$ respectively) while the remaining $k$ fields do not appear in the superpotential. Thus, for supersymmetry to remain unbroken the superpotential should be extremal with respect to $n-k$ variables, leading to $n-k$ equations in $n-k$ unknowns. Thus, generically, there is a solution and supersymmetry is unbroken.

In contrast, suppose the theory has an $R$ symmetry that is spontaneously broken. Then there is a field, $X$, with $R$ charge $q \neq 0$, which gets a non-zero vev. The superpotential then can be written as

$$
W=X^{2 / q} f\left(Y_{i}=X_{i}^{q} / X^{q_{i}}\right)
$$

where $q_{i}$ is the charge of $X_{i}$. For supersymmetry to be unbroken we need

$$
\frac{\partial f}{\partial Y_{i}}=0
$$

and

$$
f=0 \text {. }
$$

Thus there is one more equation than unknowns, and generically we do not expect a solution. Roughly speaking, what we mean by "generically" is that the superpotential is a generic function of the fields, that is, it contains all terms allowed by the symmetries. We shall return to this point shortly. 
If the extremum of the superpotential were determined by a system of homogeneous linear equations, the above discussion would lead us to conclude that an R-symmetry is a necessary condition for supersymmetry breaking, and a spontaneously broken R-symmetry is a sufficient condition. While this conclusion generally holds for theories in which the superpotential is a generic function consistent with all the symmetries, there may be exceptions to this rule. This is because the F-flatness conditions are given by a system of non-linear equations which may contain negative powers of fields (arising from the dynamical superpotential) as well as terms independent of fields (arising from linear terms, either generated dynamically or included in the tree level superpotential). Such a system of equations is not guaranteed to have solutions. In fact, we have already argued in Section IIC 1 that one can add explicit R-symmetry breaking terms to an O'Raifeartaigh model without restoring supersymmetry. Later we shall encounter other examples of supersymmetry breaking models without R-symmetry.

Let us make a bit more precise what we mean by a generic superpotential. The superpotential contains two parts. One is generated dynamically, and certainly does not contain all terms allowed by the symmetries (Seiberg, 1993). In particular, such terms could involve arbitrarily large negative powers of the fields. The other part is the classical superpotential, which is a polynomial (of some degree $d$ ) in the fields, that preserves some global symmetry. Here what we mean by "generic" is that no term, with dimension smaller or equal to $d$ that is allowed by this global symmetry was omitted from the superpotential. On the other hand, the tree-level superpotential can still be considered generic if the operators with dimension higher than $d$ are omitted. Indeed, in a renormalizable Lagrangian with a stable vacuum we do not expect Planck scale vevs. The analysis of Nelson and Seiberg (1994) shows that the inclusion of non-renormalizable operators can only produce additional minima with Planck scale vevs, that is in a region of field space where our approximation of global supersymmetry is not sufficient anyway. On the other hand, in models where a stable vacuum appears only after the inclusion of non-renormalizable terms of dimension $d$, the typical expectation values will depend on the Planck scale (or other large scale) but often will remain much 
smaller than it. As long as the expectation values are small compared to the Planck scale, these minima will remain stable local minima even if operators of dimension higher than $d$ are added.

We shall encounter several examples of theories that break supersymmetry even though they do not possess an $R$ symmetry. In some cases, while the microscopic theory does not have an $R$ symmetry, there is an effective, spontaneously broken $R$ symmetry in the low energy theory. In other cases, there is not even an effective $R$ symmetry. In one example, SUSY will be broken even though the tree-level superpotential is generic and does not preserve any $R$-symmetry, and there is no effective $R$-symmetry.

\section{Gaugino Condensation}

Let us now introduce a criterion for SUSY breaking which is based on gaugino condensation (Meurice and Veneziano, 1984; Amati-Rossi-Veneziand, 1985). Suppose that a certain chiral superfield (or a linear combination of chiral superfields) does not appear in the superpotential, yet all the moduli are stabilized. In such a case the Konishi anomaly (Konishi, 1984; Clark-Piguet-Sibold, 1979) implies

$$
\overline{\mathcal{D}}^{2}\left(\bar{\Phi} e^{V} \Phi\right) \sim \operatorname{Tr} W^{2}
$$

where $\mathcal{D}$ is a supersymmetric covariant derivative (see Appendix A 1), $\Phi$ is a chiral superfield and $V$ is the vector superfield. It is instructive to consider Eq. (20) in component form. It is given by an anomalous commutator with the supersymmetry generator $Q$,

$$
\left\{Q, \psi_{\Phi} \phi\right\} \sim \lambda \lambda
$$

where $\psi_{\Phi}$ and $\phi$ are the fermionic and scalar components of $\Phi$ respectively, and $\lambda$ is the gaugino. From this equation we see that the vacuum energy is proportional to the lowest component of $W^{2}$, that is, to $<\operatorname{Tr} \lambda \lambda>$. Therefore, if the gaugino condensate forms one

can conclude that supersymmetry is broken. Note that if the fields $\Phi$ and $\bar{\Phi}$ appear in 
the superpotential, the right-hand side of Eqs. (20) and (21) can be modified and the gaugino condensate may form without violating supersymmetry. For example, if there is a superpotential mass term, Eq. (21) becomes

$$
\left\{Q, \psi_{\Phi} \phi\right\}=m \bar{\phi} \phi+\frac{1}{32 \pi^{2}} \lambda \lambda
$$

The latter equation is compatible with supersymmetry and determines the vevs of the scalar fields in terms of the gaugino condensate.

This criterion is related to the global symmetry arguments of Affleck-Dine-Seiberg (1985), since if a gaugino condensate develops in a theory possessing an R-symmetry, this symmetry is spontaneously broken. In the absence of flat directions, the Affleck-Dine-Seiberg argument leads to the conclusion that SUSY is broken.

\section{Examples}

We shall now demonstrate the techniques described in 1 IIB and IIIC by a few examples.

\section{Spontaneously Broken Global Symmetry: the SU(5) Model}

Consider an $S U(5)$ gauge theory with one antisymmetric tensor (10) $A$, and one antifundamental $\bar{F}$ (Affleck-Dine-Seiberg, 1984b; Meurice and Veneziano, 1984). The global symmetry of the theory is $U(1) \times U(1)_{R}$, under which we can take the charges of the fields to be $A(1,1)$ and $\bar{F}(-3,-9)$. There are no gauge invariants one can make out of $A$ and $\bar{F}$. Thus there are no flat directions, and classically the theory has a unique vacuum at the origin. The theory is strongly coupled near the origin, and we have no way to determine the behavior of the quantum theory. Because there are no chiral gauge invariants, the theory

does not admit any superpotential. If supersymmetry is broken, the only possible scale for its breaking is the strong coupling scale of $S U(5)$.

Following Affleck-Dine-Seiberg (1984b) we shall now use R-symmetry to argue that supersymmetry is indeed broken (we shall consider a gaugino condensation argument due to 
Meurice and Veneziano, (1984) in the following subsection). Assuming the theory confines, the massless gauge-invariant fermions of the confined theory should reproduce the triangle anomalies generated in the microscopic theory. Affleck-Dine-Seiberg (1984b) showed that the minimal number of fermions required, with $U(1)$ and $U(1)_{R}$ charges under 50 , is five. This makes it quite implausible that the full global symmetry remains unbroken. But if the global symmetry is spontaneously broken and there are no flat directions, the theory breaks supersymmetry by the arguments of $\amalg I \mathrm{~B}$.

\section{Gaugino Condensation: the SU(5) Model}

We now would like to apply the gaugino condensate argument to the $S U(5)$ model discussed in the previous subsection. Since the gaugino condensate serves as an order parameter for supersymmetry breaking, we need to establish that it is non-zero. To do that we follow Meurice and Veneziano (1984) and consider the correlation function

$$
\Pi(x, y, z)=\left\langle T\left(\lambda^{2}(x), \lambda^{2}(y), \chi(z)\right)\right\rangle
$$

where

$$
\chi=\epsilon^{a b c d e} \lambda_{a}^{a^{\prime}} \lambda_{a^{\prime}}^{b^{\prime}} \bar{F}^{c^{\prime}} A_{b^{\prime} c^{\prime}} A_{b c} A_{d e},
$$

and $\bar{F}$ and $A$ are scalar components of $\overline{5}$ and 10 respectively.

In the limit $x, y, z \ll \Lambda^{-1}$ one can show that the condensate $\Pi \sim \Lambda^{13}$. Then one can take the limit of large $x, y$, and $z$ and using cluster decomposition properties argue that $<\lambda \lambda>\neq 0$. As a result supersymmetry must be broken.

\section{R-symmetry and SUSY breaking}

In the example of the $S U(5)$ model we could not explicitly verify the spontaneous breaking of the global symmetry, and had to rely on the use of 't Hooft anomaly matching conditions to establish supersymmetry breaking. Now we consider examples in which one 
can explicitly show that R-symmetry is spontaneously broken, and to use that to establish supersymmetry breaking. While we shall be content to only consider models with tree level breaking, similar arguments can be applied to a number of dynamical models discussed later.

Consider first the O'Raifeartaigh model of Eq. (10). There is an $R$ symmetry under which $\phi_{1}, \phi_{2}$ have $R$-charge 2 , and $\phi$ has $R$ charge zero. There is also a discrete $Z_{2}$ symmetry under which $\phi_{1}$ is neutral, while $\phi_{2}$ and $\phi$ change sign. The superpotential is the generic one consistent with the symmetries and supersymmetry is broken. We can add to the superpotential $\phi$ to some even power so that the $R$-symmetry is broken. Still, supersymmetry is broken. However, the superpotential is no longer generic, and we can add the term $\phi_{1}^{2}$ without breaking the remaining $Z_{2}$ symmetry. This term will restore supersymmetry.

If we do not wish to impose a discrete symmetry the most general superpotential is

$$
W=\sum_{i=1}^{2} M_{i}^{2} \phi_{i}+m_{i} \phi_{i} \phi+\lambda_{i} \phi_{i} \phi^{2} .
$$

The model with this superpotential still breaks supersymmetry unless $M_{1} / M_{2}=m_{1} / m_{2}=$ $\lambda_{1} / \lambda_{2}$. Note that if the parameters are chosen so that this latter equality is satisfied the superpotential is independent of one linear combination of the fields, $\tilde{\phi}=\left(m_{2} \phi_{1}-m_{1} \phi_{2}\right) /\left(m_{1}^{2}+\right.$ $\left.m_{2}^{2}\right)$, and thus is not generic.

As another example, consider the superpotential

$$
W=P_{1} X_{1}+P_{2} X_{2}+A\left(X_{1} X_{2}-\Lambda^{2}\right)+\alpha X_{1} X_{2}
$$

For $\alpha=0$ the theory has a $U(1) \times U(1)_{R}$ symmetry with $A(0,2), X_{1}(1,0), X_{2}(-1,0)$, $P_{1}(-1,2), P_{2}(1,2)$. Supersymmetry is broken whether or not $\alpha=0$. For $\alpha \neq 0$ the $R$ symmetry is broken and the potential is no longer generic. Terms such as $P_{1} P_{2}, A^{2}$, which respect the $U(1)$ could restore supersymmetry. This example is a simple version of the low energy theory of the example we shall study in section VIQ. 


\section{Generalizations of the SU(5) Model}

To conclude our presentation of the basic examples of supersymmetry breaking we construct an infinite class of models generalizing the $S U(5)$ model discussed earlier (AffleckDine-Seiberg, 1985; Meurice and Veneziand, 1984). These models have an $S U(2 N+1)$ gauge group, with matter transforming as an antisymmetric tensor $A$ and $2 N-3$ antifundamentals $\bar{F}_{i}, i=1, \ldots, 2 N-3$, Affleck-Dine-Seiberg, 1985). For $N>2$, all these models have D-flat directions, which are all lifted by the most general R-symmetry preserving superpotential

$$
W=\lambda_{i j} A \bar{F}_{i} \bar{F}_{j},
$$

for the appropriate choice of the matrix of coupling constants.

First, note that for small superpotential coupling the model possesses almost flat direc-

tions, and as a result, part of the dynamics can be analyzed directly. Yet the scale of the unbroken gauge dynamics in the low energy theory is comparable to the scale of SUSY breaking and the model is not calculable. To analyze supersymmetry breaking it is convenient to start from the theory without the tree level superpotential. In this case the models have classical flat directions along which the effective theory reduces to the $S U(5)$ theory with an antisymmetric and an antifundamental as well as the light modulus parameterizing the flat direction. We already know that in this effective theory SUSY is broken with a vacuum energy $\sim \Lambda_{L}^{4}$. The low energy scale depends on the vev of the modulus as in Eq. A21, and thus there is a potential

$$
V(\phi) \sim \Lambda_{L}^{4} \sim \phi^{-\frac{4}{13}(4 N-8)} .
$$

When small Yukawa couplings are turned on, the flat directions are stabilized by the balance between the tree level contribution of order $\lambda^{2} \phi^{4}$ and the dynamical potential (28). The minimum of the potential then occurs for

$$
\phi \sim \lambda^{-\frac{13}{2(4 N+5)}} \Lambda \quad \text { with } \quad E_{v a c} \sim \lambda^{8 \frac{N-2}{4 N+5}} \Lambda^{4} .
$$

We found that the potential is stabilized at finite value of the modulus and the effective low energy description is given in terms of the SUSY breaking theory. Thus supersymmetry 
must be broken in the full theory. We also note that at the minimum, R-symmetry is broken, giving us additional evidence for supersymmetry breaking.

Before concluding this section we comment on the analogous theories with $S U(2 N)$ gauge groups (Affleck-Dine-Seiberg (1985)). In this case the tree level superpotential allowed by symmetries (including $\mathrm{R}$ symmetry) does not lift all the classical flat directions. On the other hand, a dynamical superpotential is generated, pushing the theory away from the origin. Thus the model does not have a stable ground state. It is possible to lift all flat directions by adding R-symmetry breaking terms to the tree level superpotential. While lifting the flat directions, these terms lead to the appearance of a stable supersymmetric vacuum.

\section{DIRECT ANALYSIS: CALCULABLE MODELS}

As we have seen, SUSY breaking is directly related to the zero-energy properties of the theory, namely, the ground-state energy and the appearance of the massless Goldstino. Fortunately, then, to establish SUSY breaking, we only need to understand the low-energy behavior of the theory in question. As we shall see, many models can be described, in certain regions of the moduli space, by a low-energy O'Raifeartaigh-like effective theory. The question of whether SUSY is broken simply amounts to the question of whether all F-terms can vanish simultaneously. The tricky part, of course, is obtaining the correct low-energy theory. This involves a number of related ingredients: establishing the correct degrees of freedom, and determining the superpotential and the Kähler potential. In many cases, holomorphy and symmetries indeed determine the superpotential, but the same is not true for the Kähler potential. However, if all we care about is whether the energy vanishes or not, it suffices to know that the Kähler potential is not singular as a function of the fields that make up the low-energy theory. This, in turn, is related to whether or or not we chose the correct degrees of freedom of our low-energy theory.

We shall divide our discussion into two parts. In this section we shall consider weakly- 
coupled theories. By tuning some parameters in the superpotential to be very small, we can typically drive some of the fields to large expectation values, with the gauge symmetry completely broken, so that all gauge bosons are very heavy. We can then neglect gauge interactions, and write down, as advertised, a low-energy O'Raifeartaigh-type model. Since the theory is weakly-coupled, we shall also be able to calculate the Kähler potential, and thus, completely determine the low-energy theory including the ground-state energy, the composition of the Goldstino, and the masses of low-lying states.

But we can also, in many cases, analyze the theory near the origin in field space, where the theory is very strongly coupled. If the theory confines, then below the confinement scale, we are again left with an O'Raifeartaigh-type model. In Section $(\mathbb{\nabla})$, we shall see examples of this kind. In some cases, though we shall not be able to analyze the theory in question, we shall be able to analyze a dual theory, that, as we just described, undergoes confinement. In all these cases, however, we shall not be able to calculate the Kähler potential. Thus, while we shall ascertain that SUSY is broken, the details of the low-energy theory, and in particular the vacuum energy, the unbroken global symmetry, and the masses of low-lying states remain unknown.

In the examples we encounter, supersymmetry is broken due to a variety of effects. Still, it is always the consequence of the interplay between, on the one hand, a tree-level superpotential, which gives rise to a non-zero potential everywhere except at the origin in field space, and, on the other hand, non-perturbative effects, either in the form of instantons or gaugino condensation, that generate a potential that is non-zero at the origin.

\section{A. The 3-2 Model}

Probably the simplest model of dynamical supersymmetry breaking is the $3-2$ model of Affleck-Dine-Seiberg (1985). Here we shall choose the parameters of the model so that the low-energy effective theory is weakly coupled, and thus the model is calculable. In this weakly coupled regime, the main ingredient leading to supersymmetry breaking in the model 
is an instanton generated superpotential. In Section VIA 2, we shall analyze the same model in a strongly coupled regime where SUSY is broken through the quantum deformation of the moduli space (which is again the result of instanton effects). We shall also discuss numerous generalizations of the 3-2 model.

The model is based on an $S U(3) \times S U(2)$ gauge group with the following matter content (we also show charges under the global $U(1) \times U(1)_{R}$ symmetry of the model):

\begin{tabular}{c|cccc} 
& $S U(3)$ & $S U(2)$ & $U(1)$ & $U(1)_{R}$ \\
\hline$Q$ & 3 & 2 & $1 / 3$ & 1 \\
$\bar{u}$ & $\overline{3}$ & 1 & $-4 / 3$ & -8 \\
$\bar{d}$ & $\overline{3}$ & 1 & $2 / 3$ & 4 \\
$L$ & 1 & 2 & -1 & -3
\end{tabular}

Using methods described in Appendix A2 one can easily determine the classical moduli space of the model. In the absence of a tree level superpotential, it is given by

$$
Q_{i f}=\bar{Q}^{i f}=\left(\begin{array}{ll}
a & 0 \\
0 & b \\
0 & 0
\end{array}\right), \quad L=\left(0, \sqrt{a^{2}-b^{2}}\right)
$$

where $\bar{Q}=(\bar{u}, \bar{d})$, and $i, f$ are $S U(3)$ color and flavor indices respectively. For generic values of $a$ and $b$ the gauge group is completely broken, and there are three light chiral fields. While it is not difficult to diagonalize the mass matrix and find the light degrees of freedom, it is convenient to use an alternative parameterization of the classical moduli space in terms of the composite operators

$$
X_{1}=Q_{i \alpha} \bar{d}^{i} L_{\beta} \epsilon^{\alpha \beta}, \quad X_{2}=Q_{i \alpha} \bar{u}^{i} L_{\beta} \epsilon^{\alpha \beta}, \quad Y=\operatorname{det}(Q \bar{Q})
$$

where greek indices denote $S U(2)$ gauge indices. The most general renormalizable superpotential which preserves the $U(1) \times U(1)_{R}$ global symmetry is

$$
W_{\text {tree }}=\lambda Q \bar{d} L=\lambda X_{1}
$$

This superpotential lifts all classical flat directions. 
Let us now analyze the quantum theory. To do that we choose $\lambda^{2} \ll g_{2}^{2} \ll g_{3}^{2}$. The former inequality implies that the minimum of the scalar potential lies very close to the D-flat direction. To simplify the analysis we shall, in fact, impose D-flatness conditions. T4 The latter inequality guarantees that effects due to the $S U(2)$ non-perturbative dynamics are exponentially suppressed compared to those due to the $S U(3)$ dynamics. In particular, at scales below $\Lambda_{3}$ and much bigger that $\Lambda_{2}$, the $S U(2)$ gauge theory is weakly coupled and its dynamics can be neglected. $S U(3)$ on the other hand, confines, so we can write down an effective theory in terms of its mesons $M_{\alpha}^{f}=Q_{\alpha} \cdot \bar{Q}^{f}$, subject to the non-perturbative superpotential,

$$
W_{n p}=\frac{2 \Lambda_{3}^{7}}{\operatorname{det}(Q \bar{Q})}
$$

which is generated by an $S U(3)$ instanton. The reader may now note that in this effective theory, $S U(2)$ appears anomalous; it has three doublets, $M^{f=1,2}, L$. However, this is not too surprising, because the superpotential (34) drives the fields $Q, \bar{Q}$ away from the origin, so that $S U(2)$ is broken everywhere. Indeed, as discussed in the appendix, an $S U(3)$ gauge theory with two flavors has no moduli space.

In fact, we can already conclude that supersymmetry is broken. Since the superpotential (34) drives the fields $Q, \bar{Q}$ away from the origin, the $R$-symmetry of the model is spontaneously broken. Combining this with the fact the model has no flat directions, we see, based on the arguments of section [IIB, that the theory breaks supersymmetry.

Let us go on to analyze supersymmetry breaking in the theory in more detail. As we saw above, in the absence of a tree-level superpotential, the theory has a "runaway" vacuum with $Q, \bar{Q} \rightarrow \infty$. This is precisely what allows us to find a calculable minimum in this model. The tree-level superpotential lifts all classical flat directions. Any minimum would result from a balance between $W_{\text {tree }}$, which rises at infinity, and $W_{n p}$, which is singular at

\footnotetext{
${ }^{14}$ However, in this and in other calculable models it is easy to take D-term corrections to the scalar potential into account in numerical calculations.
} 
the origin. If we choose $\lambda$ to be very small, the minimum would occur for large $Q, \bar{Q}$ vevs, so that the gauge symmetry is completely broken, and gauge interactions are negligible. Thus for a small $\lambda \ll 1$ we can conclude that the light degrees of freedom can still be described by the gauge invariant operators $X_{1}, X_{2}$ and $Y$ (in the following we shall see additional arguments supporting the fact that $X_{1}, X_{2}$ and $Y$ are indeed the appropriate degrees of freedom). Furthermore, the superpotential in this limit is given by

$$
W=\frac{2 \Lambda_{3}^{7}}{Y}+\lambda X_{1}
$$

We now see explicitly that supersymmetry is broken, since $W_{X_{1}}=\lambda \neq 0$. Note that this conclusion depends crucially on the fact the we have the full list of massless fields. In general, one should be careful in drawing a conclusion about supersymmetry breaking based on the presence of a linear term for a composite field in the superpotential. If at some special points additional fields become massless, the Kähler metric is singular, and the potential $V=W_{i} K_{i j *}^{-1} W_{j *}$ may vanish even if all $W_{i}$ are non-zero. Moreover, if the theory has classical flat directions, it is possible that the Kähler potential (written in terms of composites) has singularities at the boundaries of moduli space, with some fields going to infinity, and possibly others to the origin. As a result supersymmetry may be restored at the origin.

As we saw above, for the choice of parameters $\Lambda_{3} \gg \Lambda_{2}, \lambda \ll 1$, the theory is weakly coupled. The Kähler potential of the low energy theory is therefore the canonical Kähler potential in terms of the elementary fields, $Q, \bar{u}, \bar{d}$ and $L$, projected on the $D$-flat direction. In terms of the gauge invariant operators it is given by (Affleck-Dine-Seiberg, 1985; BaggerPoppitz-Randall, 1994)

$$
K=24 \frac{A+B x}{x^{2}},
$$

where $A=1 / 2\left(X_{1}^{\dagger} X_{1}+X_{2}^{\dagger} X_{2}\right), B=1 / 3 \sqrt{Y^{\dagger} Y}$, and

$$
x \equiv 4 \sqrt{B} \cos \left(\frac{1}{3} \operatorname{Arccos} \frac{\mathrm{A}}{\mathrm{B}^{3 / 2}}\right) .
$$

We therefore have all the ingredients of the low energy theory, including the superpotential and the Kähler potential. This allows one to explicitly minimize the scalar potential. 
For details of the analysis, we refer the reader to Affleck-Dine-Seiberg (1985) and BaggerPoppitz-Randall (1994). Here we just give some qualitative results. It is possible to work in terms of either the elementary fields, or the gauge invariants fields. Simple dimensional analysis shows that the minimum occurs for elementary field vevs $v \sim \lambda^{-1 / 7} \Lambda_{3}$, and that the vacuum energy is of order $\lambda^{5 / 14} \Lambda_{3}$. Explicitly one finds that at the minimum $X_{2}=0$, so that the global $U(1)$ symmetry is unbroken (not surprising, as points of higher symmetry are extremal). The massless spectrum contains the Goldstino, a massless fermion of $U(1)$ charge -2 , which saturates the $U(1)$ anomaly, as well as a massless scalar which is the Goldstone boson of the broken $R$-symmetry (usually known as the $R$-axion).

This concludes our discussion of the calculable minimum of the 3-2 model, but let us make a few more comments.

First, the above analysis of supersymmetry breaking did not involve the strong dynamics of $S U(2)$. It is interesting to see therefore the effect of turning off the $S U(2)$ gauge interactions. We then have an $S U(3)$ gauge theory with two flavors, plus two singlets $L_{\alpha=1,2}$, and with the superpotential (33). Classically, this superpotential leaves a set of flat directions. Up to global symmetry transformations (which now include an $S U(2)$ global symmetry), these flat directions are parameterized by $L_{1}$ and $Q_{2} \bar{u}$. The nonperturbative dynamics leads to runaway towards a supersymmetric vacuum at infinity along this direction. 四 This dangerous direction is no longer $D$-flat when the $S U(2)$ is turned on.

Second, even though so far we concentrated on the limit $\Lambda_{3} \gg \Lambda_{2}$, it is possible to derive the exact superpotential of the 3-2 model for any choice of couplings, and to use it to establish supersymmetry breaking. Note first that the complete list of independent gauge invariants is $X_{1}, X_{2}, Y$ and $Z \equiv Q^{3} L$ (we suppress all indices). The latter vanishes classically, or more precisely in the limit $\Lambda_{2} \rightarrow 0$. In the limit $\Lambda_{3} \gg \Lambda_{2}, \lambda=0$, the superpotential is

\footnotetext{
${ }^{15}$ We shall discuss the quantum behavior of SUSY QCD coupled to singlet fields in detail in Section VIA 1 .
} 
given by (34). In the limit $\Lambda_{2} \gg \Lambda_{3}, \lambda=0$, the theory is an $S U(2)$ gauge theory with two flavors and a quantum constraint which can be implemented in the superpotential using a Lagrange multiplier $A$, as $A\left(Z-\Lambda_{2}^{4}\right)$. The most general superpotential that respects all the symmetries of the theory is

$$
W=\frac{2 \Lambda_{3}^{7}}{Y} f\left(t, z^{\prime}\right)+A\left(Z-\Lambda_{2}^{4}\right) g\left(t, z^{\prime}\right)
$$

where $t \equiv \lambda X_{1} Y / \Lambda_{3}^{7}, z^{\prime} \equiv Z / \Lambda_{2}^{4}$ are the only dimensionless field combinations neutral under all symmetries. In the limit $\Lambda_{3}, \lambda \rightarrow 0$ (for which any value of $t$ can be attained) we find an $S U(2)$ theory with 4 doublets (and a set of non-interacting singlet fields). Since the exact superpotential for this theory is known we find $g\left(t, z^{\prime}\right) \equiv 1$. Similarly, in the limit $\Lambda_{2} \rightarrow 0$ we find $f\left(t, z^{\prime}\right)=1+t$. The exact superpotential is then

$$
W=\frac{2 \Lambda_{3}^{7}}{Y}+A\left(Z-\Lambda_{2}^{4}\right)+\lambda X_{1}
$$

It is clear from this superpotential that $Z$ obtains a mass. To see if the mass is large one needs to know the Kähler potential for this field. In the limit of weakly coupled $S U(2)$ and $\lambda \ll 1$ both gauge groups are strongly broken, and the Kähler potential is close to the classical one. Since classically $Z$ vanishes, the projection of the classical Kähler potential on it also vanishes (see Eq. (36)). For small, but non-vanishing $\Lambda_{2}$ the Kähler potential of $Z$ is suppressed by some function of $\Lambda_{2} / v$. Restoring the canonical normalization for the kinetic term we find that the mass of $Z$ is enhanced by the inverse of this function. We were therefore justified in keeping only $X_{1}, X_{2}$ and $Y$ as the light fields.

Looking at (39), we can conclude that supersymmetry is broken for any choice of the parameters of the theory. However, unlike in the limit $\Lambda_{3} \gg \Lambda_{2}$, in general the theory is strongly coupled, and we have no control over the Kähler potential. Therefore, while we may be able to estimate the scale of supersymmetry breaking we cannot say anything about the vacuum, e. g. we cannot establish the pattern of symmetry breaking.

\footnotetext{
${ }^{16}$ Actually, with $S U(3)$ turned off, $Z$ in this superpotential should be understood as the Pfaffian of the $S U(2)$ mesons.
} 
In the above, we first showed that supersymmetry is broken in a specific limit, and later realized that it is always broken. Indeed, we do not expect a theory to break supersymmetry for some choice of parameters, and to develop a supersymmetric minimum for other choices. The reason is that no phase transitions are expected to occur in supersymmetric theories as their parameters are varied (Seiberg and Witten, 1994a; Seiberg and Witten, 1994b; Intriligator and Seiberg, 1994). If a theory is supersymmetric for some choice of parameters, it remains supersymmetric for any choice. This allows us to establish supersymmetry breaking by considering a convenient limit. In some theories, including the 3-2 model, we can establish supersymmetry breaking in different limits. The details of supersymmetry breaking, such as the vacuum energy and the source of the breaking, may be very different in the different limits.

Finally, another interesting feature of the $3-2$ model is the possibility of gauging the global $U(1)$ symmetry, provided that a new field $E^{+}$, with $U(1)$ charge +2 , is added to cancel the $U(1)^{3}$ anomaly. With the addition of this field, the analysis of dynamical supersymmetry breaking does not change since neither new classical flat directions appear nor are new tree level superpotential terms allowed. This possibility proved to be useful in phenomenological model building (Dine-Nelson-Shirman, 1995). For our purposes, however, the importance of this $U(1)$ is in the observation (Dine et a, 1996 ) that with the addition of $E^{+}$, the matter content of the model falls into complete $S U(5)$ representations, and in fact they are the same representations which are required for DSB in the $S U(5)$ model of Section IIID. In the following section we shall discuss another simple and calculable model of DSB based on an $S U(4) \times U(1)$ gauge group and again see that the matter fields form complete $S U(5)$ representations. In Section $\mathrm{VIIA}$ we shall introduce a method of constructing large classes of DSB models based on this observation. This method will lead us to an infinite class of models generalizing 3-2 model. Many other calculable and non-calculable generalizations will be discussed in Section VTI. 


\section{B. The 4-1 Model}

Another example of a calculable DSB model is the $4-1$ model constructed by Dine et a (1996) and Poppitz and Trivedi (1996). Consider an $S U(4) \times U(1)$ gauge group with matter transforming as an antisymmetric tensor of $S U(4), A_{2}$ (where the subscript indicates $U(1)$ charge), a fundamental, $F_{-3}$, an anti-fundamental, $\bar{F}_{-1}$, and an $S U(4)$ singlet, $S_{4}$.

For a range of parameters of the model, the scale of the gauge dynamics will be below the SUSY breaking scale. Thus one could analyze supersymmetry breaking in terms of the microscopic variables (Dine et aq, 1996). Indeed, in terms of the microscopic variables the Kähler potential of the light degrees of freedom is nearly canonical and it is easy to calculate the vacuum energy. We shall, however, analyze this model in terms of the gauge invariant polynomials. Again for convenience we shall work in a regime where the couplings are arranged hierarchically, with the superpotential Yukawa coupling the smallest, and with the $U(1)$ coupling weak at the $S U(4)$ strong coupling scale $\square$. The $S U(4)$ moduli space is given by the fields $M=F \bar{F}, X=\operatorname{Pf} A$, and $S$. The model possesses a non-anomalous R-symmetry, and the unique superpotential allowed by the symmetries is

$$
W=\frac{\Lambda_{4}^{5}}{\sqrt{M X}}+\lambda S M
$$

The tree level term in the superpotential lifts all classical flat directions (note that one more condition on the $S U(4)$ moduli is imposed by the $U(1) \mathrm{D}$-term). Due to the non-perturbative superpotential the vacuum cannot lie in the origin of the moduli space of the theory. As a result, the R-symmetry is spontaneously broken at the minimum of the potential and supersymmetry is broken.

Let us argue that the non-perturbative term in the superpotential (40) is indeed generated. We would also like to establish that the model is calculable, namely, that for some

\footnotetext{
${ }^{17}$ Since both Yukawa and $U(1)$ couplings become weaker in the infrared we can just choose them to be sufficiently small in the ultraviolet.
} 
choices of parameters, the corrections to the classical Kähler potential are small near the minimum. To this end, neglect the tree level superpotential and consider a region of the classical moduli space with $M, S^{2} \gg X$. In this region the gauge group is broken down to an $S U(3)$ subgroup. Apart from the light modulus which controls the scale of the unbroken gauge group there is one $S U(3)$ flavor in the fundamental representation coming from the components of $A$. In this effective theory the non-perturbative superpotential is generated by gaugino condensation

$$
W=\frac{\Lambda_{3}^{4}}{\sqrt{q \bar{q}}},
$$

where $q$ and $\bar{q}$ denote light $S U(3)$ fields, and $\Lambda_{3}^{4}$ is an $S U(3)$ scale. Using the scale matching condition

$$
\Lambda_{3}^{4}=\frac{\Lambda_{4}^{5}}{\sqrt{M}},
$$

we easily recognize the superpotential (40).

Furthermore, we see that the effective $S U(3)$ also possesses a flat direction along which $q$ and $\bar{q}$ acquire vevs and the gauge group is broken down to $S U(2)$. The strong scale of this $S U(2)$ tends to zero along the flat direction. While the tree level superpotential stabilizes the theory at finite vevs, the corrections to the classical scalar potential which scale as $\Lambda_{2} / v$ (where $v$ is the typical vev) are negligible for sufficiently small $\lambda$ and therefore the model is calculable. The vacuum energy in this model was explicitly calculated by Dine et at (1996).

It is worth noting that one can add an R-symmetry breaking (and non-renormalizable) term, $M \operatorname{Pf} A$, to the superpotential (40) (Poppitz and Trived, 1996). We shall discuss DSB models without R-symmetry in more detail in Section VIQ.

\section{DIRECT ANALYSIS: STRONGLY-COUPLED THEORIES}

\section{A. Supersymmetry Breaking Through Confinement}

In previous sections we have seen that, at the classical level, supersymmetric gauge theories without explicit mass terms possess a zero energy minimum at least at the origin of 
field space. Classical tree level superpotentials may lift the moduli space, but the supersymmetric vacuum at the origin survives. In traditional calculable models of DSB (such as the 3-2 model) the vacuum at the origin is lifted due to a dynamical superpotential generated by non-perturbative effects. On the other hand, in the $S U(5)$ model, no superpotential can be generated, and supersymmetry is broken by the confining dynamics. Unfortunately, the low energy spectrum of the $S U(5)$ model is not known, and thus the main arguments for DSB are based on the complexity of the solutions to the 't Hooft anomaly matching conditions. It would be very useful to investigate a model in which supersymmetry breaking is generated by the confining dynamics, with a known low energy spectrum. In fact a very simple and instructive model of this type was constructed by Intriligator-Seiberg-Shenker (1995). This model clearly illustrates the fact that a crucial ingredient in studying supersymmetry breaking is the knowledge of the correct degrees of freedom of the low-energy theory. Supersymmetry breaking in this theory hinges on whether the theory confines, or has an interacting Coulomb phase at the origin. It seems very plausible that the theory indeed confines, and that supersymmetry is broken as a result.

The model is based on an $S U(2)$ gauge theory with a single matter field, $q_{\alpha \beta \gamma}$, in a three-index symmetric representation. The model is chiral, since the quadratic invariant, $q^{2}$, vanishes by the Bose statistics of the superfields. It also possesses an R-symmetry under which $q$ has the charge 3/5. Moreover, the model is asymptotically free, and thus non-trivial infra-red dynamics may lift the supersymmetric vacuum at the origin of field space. It is, therefore, a candidate model of DSB according to traditional criteria for supersymmetry breaking.

The only non-trivial gauge invariant polynomial which can be constructed out of $q$ is $u=q^{4}$ (with appropriate contraction of indices). This composite parametrizes the only flat direction of the theory along which the gauge group is completely broken. R-symmetry and holomorphy restrict any effective superpotential to be of the form $W=a \Lambda^{-1 / 3} u^{5 / 6}$, where $\Lambda$ is the dynamical scale of the theory. This superpotential, however, does not have a sensible behavior as $\Lambda \rightarrow 0$, since for large $u / \Lambda^{4}$ the moduli space should be close to the 
classical one with $W \rightarrow 0$. Therefore, $a=0$. This means that the quantum theory also has a moduli space of degenerate vacua. The moduli space may be lifted by the tree level non-renormalizable superpotential

$$
W=\frac{\lambda}{M} u
$$

where $\lambda$ is a constant of order 1 . In the presence of the nonrenormalizable term, the model can be thought of as a low energy effective description of a more fundamental theory, which is valid below the scale $M$. Choosing $\Lambda \ll M$, there is a region of moduli space, with $\Lambda^{4} \ll u \ll M^{4}$, in which the gauge dynamics is weak and we have a good description of the physics in terms of an effective theory of chiral superfields.

In the presence of the non-renormalizable term, holomorphy and symmetries restrict the exact superpotential to be

$$
W=\frac{\lambda}{M} u f\left(t=\Lambda^{2} u / M^{6}\right)
$$

where the function $f$ is given by the sum of instanton contributions. In the allowed region, $|t| \ll 1, f \approx 1$ and we can use the classical superpotential.

Naively, the linear superpotential for $u$ leads to supersymmetry breaking since $F_{u} \neq 0$. One should remember, however, that as $u$ is a composite field, its Kähler potential may be quite complicated. In particular, the Kähler potential may be singular at some points in the moduli space, potentially leading to supersymmetry restoration.

To determine the behavior of the Kähler potential, consider first the theory for large expectation values of $u$. In this regime the description of the model should be semiclassical and thus the Kähler potential scales as

$$
\mathcal{K} \sim Q^{\dagger} Q \sim\left(u^{\dagger} u\right)^{1 / 4}
$$

Indeed this Kähler potential is singular at $u=0$. The singularity reflects the fact that at $u=$ 0 the gauge bosons become massless and must be included in the effective description. There are two plausible alternatives for the nature of the singularity in the quantum theory. It is 
possible that the theory is in a non-abelian Coulomb phase. On the other hand, it is possible that the singularity is smoothed out quantum mechanically. nntriligator-Seiberg-Shenken (1995) argued that this latter option is probably realized since the massless composite field $u$ satisfies the 't Hooft anomaly matching conditions, which is quite non-trivial.to We shall assume that this is indeed the case. Then R-symmetry, smooth behavior near the origin and semiclassical behavior at infinity imply that the Kähler potential is a (smooth) function of $u^{\dagger} u /|\Lambda|^{8}$ satisfying

$$
K=|\Lambda|^{2} k\left(u^{\dagger} u /|\Lambda|^{8}\right) \sim \begin{cases}u^{\dagger} u /|\Lambda|^{6}, & u^{\dagger} u \ll \Lambda^{8} \\ \left(u^{\dagger} u\right)^{1 / 4}, & u^{\dagger} u \gg \Lambda^{8} .\end{cases}
$$

Combining this form of the Kähler potential with the superpotential of Eq.(44) (with $f \equiv 1$ ) we find that the scalar potential

$$
V=\left(K_{u^{\dagger} u}\right)^{-1}\left|W_{u}\right|^{2}=\left(K_{u^{\dagger} u}\right)^{-1}\left|\frac{\lambda}{M}\right|^{2}
$$

necessarily breaks supersymmetry with a vacuum energy of order

$$
E \sim \frac{|\Lambda|^{6}}{M^{2}}
$$

At this point we should comment on several other interesting properties of the model. Before adding the tree-level superpotential (43), the effective description of the confined theory in terms of the $u$ modulus possesses an accidental global $U(1)$ symmetry (as the Kähler potential does not depend on the phase of $u$ ). This $U(1)$ is anomalous in terms of the elementary degrees of freedom. The tree level superpotential explicitly breaks the Rsymmetry of the model, as well as the accidental $U(1)$. However in the low energy description there is an effective R-symmetry which is a combination of the $U(1)_{R}$ and accidental $U(1)$ symmetries. Since R-symmetry in the macroscopic description is explicitly broken by the tree

\footnotetext{
${ }^{18}$ See, however, (Brodie-Cho-Intriligator, 1998), for a class of theories in which the existence of simple solutions to the anomaly matching conditions suggests that the theories confine, yet the theories in fact do not confine.
} 
level superpotential, higher order terms can generically correct (43). These terms explicitly violate the effective R-symmetry of the low energy description. According to our analysis in section IIIB this leads to the appearance of supersymmetric vacua, but these vacua will lie outside the region of validity $|u|<M^{4}$ of our analysis, and the non-supersymmetric minimum will remain a (metastable) local minimum of the potential.

\section{B. Establishing Supersymmetry Breaking through a Dual Theory}

In this section we shall encounter a class of theories, with $S U(N) \times S U(N-2)$ gauge symmetry, that break supersymmetry for odd $N$. By directly studying these theories, we can show that they have calculable, supersymmetry-breaking minima for a certain choice of parameters. But we cannot show that there is no supersymmetric minimum, simply because we cannot analyze the low-energy theory in all regions of the moduli space. However, we shall be able to construct a Seiberg-dual of the original theory, that can be reliably analyzed at low-energy. As we shall see, the dual theory breaks supersymmetry. We can then conclude that the original theory breaks supersymmetry as well. The reason is, that at least as long as supersymmetry is unbroken, the two duals should have the same physics at zero energy. It is therefore impossible for one of them to be supersymmetric, with a vacuum at zero energy, and for the other one to break supersymmetry, with non zero energy vacuum.

Furthermore, it is possible that the two dual theories actually agree not just at zero energy, but in a small, finite energy window. In the theories at hand, the scale of supersymmetry breaking is proportional to some superpotential coupling, and can be tuned to be small enough so that it is within this energy window. It is important to stress however that we only use the dual to establish that supersymmetry is broken. The details of supersymmetry breaking may be different between the original theory and its dual.

While we mostly concentrate on the application of duality to establish supersymmetry breaking, one could adopt a different point of view, and use duality to construct new models of DSB starting with known models. Generically new models constructed in such a way will 
describe completely different, yet non-supersymmetric infrared physics.

Let us turn now to our example. The theory we start with is an $S U(N) \times S U(N-2)$ $(N \geq 5)$ gauge theory with fields $Q_{i \alpha}$, transforming as $(N, N-2)$ under the gauge groups, $N-2$ fields, $\bar{L}_{I}^{i}$, transforming as $(\bar{N}, \mathbf{1})$, and $N$ fields, $\bar{R}_{A}^{\alpha}$, that transform as $(\mathbf{1}, \overline{N-2})$. We denote the gauge indices of $S U(N)$ and $S U(N-2)$ by $i$ and $\alpha$, respectively, while $I=1 \ldots N-2$ and $A=1 \ldots N$ are flavor indices. Note that these theories are chiral-no mass terms can be added for any of the matter fields.

In the following, we shall only outline the main stages of the analysis. For details we refer the reader to (Poppitz-Shadmi-Trivedi, 1996b). In particular, we omit numerical factors and some scale factors throughout this section.

The classical moduli space of the theory is given by the gauge invariants $Y_{I A}=\bar{L}_{I} \cdot Q \cdot \bar{R}_{A}$, $\bar{b}^{A B}=\left(\bar{R}^{N-2}\right)^{A B}$ and $\overline{\mathcal{B}}=Q^{N-2} \cdot \bar{L}^{N-2}$ (when appropriate, all indices are contracted with $\epsilon$-tensors), subject to the classical constraints $Y_{I A} \bar{b}^{A B}=0$ and $\bar{b}^{A B} \overline{\mathcal{B}} \sim\left(Y^{N-2}\right)^{A B}$.

To lift all classical flat directions, we can add the superpotential,

$$
W_{\text {tree }}=\lambda^{I A} Y_{I A}+\alpha_{A B} \bar{b}^{A B},
$$

with $\lambda_{I A}=\lambda \delta_{I A}$ for $A \leq N-2$, and zero otherwise. $\alpha_{A B}$ is an antisymmetric matrix, whose non-zero elements are $\alpha_{12}=\ldots=\alpha_{(N-2)(N-1)}=\alpha$ for odd $N$, and $\alpha_{12}=\ldots=\alpha_{(N-1) N}=\alpha$ for even $N$. 可 Note that the second term in (49) is non-renormalizable for $N \geq 6$, but has dimension four for $N=5$.

As it turns out, there is an important difference between the theories with even and odd $N$. For odd $N$, the superpotential (49) preserves an $R$ symmetry, and one may expect supersymmetry to break. For even $N$, there is no $R$ symmetry that is preserved by (49), so supersymmetry is most likely unbroken . Both of these statements are indeed borne out by direct analysis, as we shall see.

\footnotetext{
${ }^{19}$ In fact, one can lift all flat directions with other choices for $\lambda^{I A}$ and $\alpha_{A B}$, see Poppitz-ShadmiTrivedi (1996b).
} 
It is also easy to check that if we set $\alpha_{A B}=0$ in (49), all flat directions are lifted, except for the "baryon" directions $\bar{b}^{A B}$.

To analyze the quantum theory, we can start with the limit $\Lambda_{N} \gg \Lambda_{N-2}$, where $\Lambda_{N}, \Lambda_{N-2}$ are the strong coupling scales of $S U(N), S U(N-2)$ respectively. $S U(N)$ has $N-2$ flavors, so gaugino condensation in an unbroken $S U(2)$ subgroup generates the superpotential

$$
W \sim\left(\frac{\Lambda_{N}^{2 N+2}}{\overline{\mathcal{B}}}\right)^{1 / 2}
$$

Thus there is no moduli space. Below $\Lambda_{N}, S U(N-2)$ appears anomalous. It is also partially broken. This is reminiscent of the situation we encountered in the 3-2 model. However, there, because of the $S U(3)$ superpotential, the $S U(2)$ was completely broken. In contrast, here the $S U(N-2)$ is not completely broken, so there is some strong dynamics associated with the unbroken group. It is therefore very hard (or impossible) to analyze the theory (except for a special choice of parameters, for which it has a calculable minimum, as we shall see later). Fortunately, we can turn to a dual theory, in which the low-energy dynamics is under control. 0

Before we do that, one comment is in order. It is already clear from Eq. (50) that the electric theory has no moduli space. In addition, with $\alpha=0$, the theory has classical flat directions. If these are not lifted quantum mechanically, the superpotential (50) pushes some fields to large vevs along these directions. Then for $\alpha \ll 1$ we can find a calculable minimum. This indeed is the case. We shall return to this calculable minimum towards the end of the section. First, however, we would like to show that the theory has no supersymmetric vacua. To do that, we turn to the dual theory.

We construct the dual theory in the limit $\Lambda_{N-2} \gg \Lambda_{N}$. However, it is expected to give a valid description of the original theory in the infrared for any $\Lambda_{N-2} / \Lambda_{N}$ (Poppitz-ShadmiTrivedi, 1996a). The dual theory is obtained by dualizing the $S U(N-2)$. This can be

\footnotetext{
${ }^{20}$ The appearance of the superpotential (50) can be seen in the dual theory as well (PoppitzShadmi-Trivedi, 1996b).
} 
thought of as the process of first turning off the $S U(N)$ coupling, so that we are left with an $S U(N-2)$ with $N$ flavors. Dualizing this theory we find an $S U(2)$ theory with $N$ flavors. Finally, we switch the $S U(N)$ coupling back on in this dual theory.

The dual theory then has $S U(N) \times S U(2)$ gauge symmetry, with the following field content:

\begin{tabular}{c|cc} 
& $S U(N)$ & $S U(2)$ \\
\hline$q_{\nu}^{i}$ & $\bar{N}$ & 2 \\
$\bar{r}^{A \nu}$ & 1 & 2 \\
$\frac{1}{\mu} M_{i A}$ & $N$ & 1 \\
$\bar{L}_{I}^{i}$ & $\bar{N}$ & 1
\end{tabular}

The $S U(2)$ singlets $M_{i A}$ correspond to the $S U(N-2)$ mesons $q_{i} \cdot R_{A}$, and $\mu$ is a mass scale that relates the strong coupling scales of $S U(N-2)$ and $S U(2): \Lambda_{N-2}^{2 N-6} \Lambda_{2}^{6-N} \sim \mu^{N}$.

In addition, the dual theory has a Yukawa superpotential:

$$
W=\frac{1}{\mu} M_{\alpha A} \bar{r}^{A} \cdot q^{\alpha} .
$$

Note that in this dual theory, $S U(2)$ has $N$ flavors, so naively it is in the dual regime. We shall soon see however that the combination of the Yukawa superpotential (52) and the $S U(N)$ dynamics, drives the theory into the confining regime.

To see that, note that $S U(N)$ now has $N$ flavors, and therefore, a quantum modified moduli space. Below the $S U(N)$ confining scale W, we can write down an effective theory in terms of the $S U(N)$ mesons $N_{A \nu} \sim M_{i A} q_{\nu}^{i}$ and $Y_{I A} \sim M_{i A} \bar{L}_{I}^{i}$, and the $S U(N)$ baryons $\mathcal{B} \sim \operatorname{det}\left(\mathcal{M}_{\alpha \mathcal{A}}\right)$ and

$$
\overline{\mathcal{B}}^{\prime} \sim q^{2} \cdot \bar{L}^{N-2} \sim Q^{N-2} \cdot \bar{L}^{N-2} \sim \overline{\mathcal{B}}
$$

where in the last equation we used the baryon map of SQCD, Eq. (A38). Here we omit various scales as well numerical coefficients.

\footnotetext{
${ }^{21}$ This scale is not $\Lambda_{N}$. Rather, it is a combination of $\Lambda_{N}, \Lambda_{N-2}$, and $\mu$ Poppitz-ShadmiTrivedi, 1996b).
} 
In terms of these variables, the $S U(2)$ still has $2 N$ doublets, $N_{A}$ and $\bar{r}^{A}$, but the superpotential (52) now turns into

$$
W \sim N_{A} \cdot \bar{r}^{A}
$$

which gives masses to all $S U(2)$ doublets. Thus, indeed, as $S U(N)$ confines, the Yukawa couplings turn into mass terms and drive $S U(2)$ into the confining regime.

Since $S U(2)$ is now confining, with mass terms for all its doublets, we should work in terms of its mesons, all of which obtain vevs. A convenient way of keeping track of the correct vevs is to add the superpotential

$$
W \sim\left(\frac{\operatorname{Pf} V}{\Lambda_{2 L}^{6-N}}\right)^{\frac{1}{N-2}} .
$$

Here $\Lambda_{2 L}$ is the $S U(2)$ scale after $S U(N)$ confines, and $V$ stands collectively for the $S U(2)$ mesons $\left[N^{2}\right],\left[\bar{r}^{2}\right],[N \cdot \bar{r}]$. We use brackets to indicate that these mesons should be thought of as single fields now.

In addition, recall that the $S U(N)$ dynamics leads to a constraint that can be implemented through the superpotential

$$
A\left(\left[N^{2}\right] \cdot Y^{N-2}-\mathcal{B} \overline{\mathcal{B}}-\bar{\Lambda}_{N L}^{2 N}\right)
$$

where $A$ is a Lagrange multiplier, and $\bar{\Lambda}_{N L}$ is the $S U(N)$ scale.

Combining (55), (54) and (53) with the tree level superpotential, which now has the form

$$
\lambda^{I A} Y_{I A}+\alpha_{A B}\left[\bar{r}^{A} \cdot \bar{r}^{B}\right]
$$

we have the complete superpotential. Note that in the last step we used the SQCD baryon $\operatorname{map} \bar{b}^{A B} \sim \bar{r}^{A} \cdot \bar{r}^{B}$.

We now have a low energy field theory with all gauge dynamics integrated out. This low energy theory consists of the fields $\mathcal{B}, \overline{\mathcal{B}}, Y_{I A},\left[N^{2}\right],\left[r^{2}\right]$ and $[N \cdot r]$, with a superpotential that is given by adding (53) through (56). We can check then whether all $F$ terms vanish simultaneously. This is a rather tedious task, and we refer the interested reader 
to (Poppitz-Shadmi-Trivedi, 1996b). As the analysis shows, for odd $N$, no solution exists, and supersymmetry is broken. For even $N$ a solution does exist.

It is interesting to see what happens before adding the tree-level superpotential. In that case, the $F$ equations have no solution for finite field vevs. Furthermore, for $\alpha=0$ and $\lambda \neq 0$, the different $\mathrm{F}$-terms tend to zero as some of the baryons $\bar{b}^{A B}$ tend to infinity.

One difficulty which we glossed over in the above discussion is related to the fact that after $S U(N)$ confines, the $S U(2)$ scale $\Lambda_{2 L}$ is field dependent. If this scale vanishes, additional fields may become massless, and the Kähler potential in terms of the degrees of freedom we kept so far may become singular. To resolve this issue, one can add a heavy $S U(N)$ flavor. In this case, no scale is field dependent, and the analysis confirms the results stated above. In particular, one finds that supersymmetry is broken for odd $N$. For further details see (Poppitz-Shadmi-Trivedi, 1996b).

To summarize, while we could not in general analyze the original $S U(N) \times S U(N-2)$ theory, we were able to show that it breaks supersymmetry for odd $N$ by studying its dual $S U(N) \times S U(2)$ theory.

To complete our discussion of this theory, we now turn to the calculable minimum we mentioned earlier. This minimum can be studied in the electric theory itself, so duality plays no role in the analysis.

As we already mentioned, with $\alpha=0$, all $F$ terms asymptote to zero along the baryonic flat direction. Let us now see this in the electric theory. We choose a particular baryon direction, with $R_{A}^{i}=v \delta_{A}^{i}$. This corresponds to $\bar{b}^{(N-1) N} \sim v^{N-2}$, with all other $\bar{b}^{A B}=0$. Along this direction, $S U(N-2)$ is completely broken, so for large $v$ we can neglect its effects. Furthermore, the first term in (49) gives masses $\lambda v$ to all $S U(N)$ flavors (in the following we set $\lambda=1$ for convenience). At low energies we are thus left with a pure $S U(N)$, whose scale $\Lambda_{N L}$ satisfies $\Lambda_{N L}^{3 N} \sim v^{N-2} \Lambda_{N}^{2 N+2}$. Gaugino condensation in this theory then leads to a superpotential

$$
W \sim\left(v^{N-2}\right)^{1 / N} \sim\left(\bar{b}^{(N-1) N}\right)^{1 / N}
$$


We thus have a low energy theory in terms of the baryons $\bar{b}^{A B}$, with the superpotential (57) (for $\alpha=0$ ), so the $F$ term for $\bar{b}^{(N-1) N}$ behaves as $F \sim\left(\bar{b}^{(N-1) N}\right)^{\frac{1}{N}-1}$ which goes to zero as $\bar{b}^{(N-1) N} \rightarrow \infty$. But whether this is a runaway direction or not depends on the Kähler potential. In fact, it can be argued (Shirman, 1996) that the Kahler potential is canonical in terms of the elementary fields $\bar{R}^{A}$, up to small corrections. Thus, if the $F$ terms for $\bar{R}^{A}$ tend to zero along this direction, there is a runaway minimum at infinity. Indeed, these $F$ terms behave as $v^{\frac{N-2}{N}-1}=v^{-2 / N}$ and asymptote to zero as $v \rightarrow \infty$.

Adding now a small $\alpha \neq 0$, the potential can be stabilized as $v \rightarrow \infty$, with a supersymmetry-breaking minimum for large values of $v$. Note that for $N \geq 6$, the baryon term in (49) is non-renormalizable, so $\alpha$ is naturally small. In fact, as was shown in (Poppitz and Trivedi, 1997), this minimum can be analyzed using a simple $\sigma$-model approach, and is interesting for model building purposes, as there is a large unbroken global symmetry at the minimum, in which, a priori at least, one can embed the standard-model gauge group.

\section{Integrating Matter In and Out}

In Section III D we discussed the supersymmetry breaking $S U(5)$ model with matter in the antisymmetric tensor and in the antifundamental representations. This model does not possess any classical or dynamical superpotential and does not have flat directions. We gave two arguments establishing DSB. One was based on the complexity of solutions to 't Hooft anomaly matching conditions, while the other was based on the formation of the gaugino condensate. We also discussed generalizations of the $S U(5)$ model.

Here we shall use the same class of theories to illustrate another method of analysis which is useful in non-calculable models (Murayama, 1995; Poppitz and Trivedi, 1996). In this method one modifies the model of interest to make it calculable through the introduction of extra vector-like matter. When these vector-like matter fields are massless, the models typically posses flat directions along which the gauge group is broken and the theory is in a weak coupling regime. For small masses of these matter fields the weak coupling 
approximation is still reliable and the theory remains calculable. Thus, the modified theory with small masses allows a direct analysis of supersymmetry breaking. Then one considers the limit of infinite vector-like matter mass. In this limit the vector-like matter decouples and one is left with the original theory. If supersymmetry is broken in the modified theory with the additional light fields, holomorphy arguments ensure that it is broken for any finite values of masses. Moreover, since the theories that we have in mind do not have classical flat directions both for finite and infinite masses, we expect that the asymptotic behavior of the scalar potential (and therefore, the Witten index) remain unchanged in the infinite mass limit. Thus the assumption that no phase transition occurs when going to the infinite mass limit, leads us to the conclusion that supersymmetry is broken in the original strongly coupled model. We stress that this approach gives strong evidence for supersymmetry breaking, yet does not help in understanding the strongly coupled SUSY breaking vacuum. The reason is that as the mass of the vector-like matter becomes large, $m \sim \Lambda$, control of the Kähler potential is lost and the theory becomes non-calculable.

We now discuss the application of this method to the models at hand, following Poppitz and Trivedi (1996). We consider models with an $S U(2 N+1)$ gauge group, an antisymmetric tensor $A_{\alpha \beta}, 2 N-3+N_{f}$ antifundamentals $\bar{Q}_{i}^{\alpha},\left(i=1, \ldots, 2 N-3+N_{f}\right)$, and $N_{f}$ fundamentals $Q_{\alpha}^{a},\left(a=1, \ldots, N_{f}\right)$. It is convenient to start with the case $N_{f}=3$, and then to integrate out vector-like matter. The classical moduli space is described by the following gauge invariant operators

$$
\begin{aligned}
& M_{i}^{a}=\bar{Q}_{i}^{\alpha} Q_{\alpha}^{a}, \\
& X_{i j}=A_{\alpha \beta} \bar{Q}_{i}^{\alpha} \bar{Q}_{j}^{\beta}, \\
& Y^{a}=\epsilon^{\alpha_{1}, \ldots, \alpha_{2 N+1}} A_{\alpha_{1}, \alpha_{2}} \cdots A_{\alpha_{2 N-1} \alpha_{2 N}} Q_{\alpha_{2 N+1}}^{a}, \\
& Z=\epsilon^{\alpha_{1}, \ldots, \alpha_{2 N+1}} A_{\alpha_{1} \alpha_{2}} \cdots A_{\alpha_{2 N-3,2 N-2}} Q_{\alpha_{2 N-1}}^{a} Q_{\alpha_{2 N}}^{b} Q_{\alpha_{2 N+3}}^{c} \epsilon_{a b c} .
\end{aligned}
$$

These moduli overcount by one the number of the massless degrees of freedom at a generic point of the moduli space, and thus are related by a single constraint which easily follows from the Bose statistics of the superfields 


$$
Y \cdot M^{2} \cdot X^{N-1}-\frac{k}{3} Z \operatorname{Pf} X=0
$$

where appropriate contraction of indices is assumed. Vevs of the moduli (58) satisfying the constraint (59) describe non-equivalent classical vacuum states. The Kähler potential of the theory written in terms of the gauge invariant composites is singular at the origin. As usual this singularity reflects the fact that the gauge symmetry is restored at the origin of the moduli space and additional massless degrees of freedom descend into the low energy theory. In complete analogy with SQCD (see Eq. (A28)) this constraint is modified by non-perturbative effects,

$$
Y \cdot M^{2} \cdot X^{N-1}-\frac{k}{3} Z \operatorname{Pf} X=\Lambda^{4 N+2}
$$

As a result, the origin of field space where the gauge symmetry is completely restored does not belong to the quantum moduli space. The Kähler potential is non-singular in any finite region of the moduli space, and we have good control of the physics. We note in passing that the Kähler potential may still become singular at the boundaries of the (D-flat) moduli space, where a subgroup of the original $S U(2 N+1)$ gauge group remains unbroken (corresponding to the situation with some moduli vevs vanishing while other vevs tend to infinity). We shall carefully consider models in which the physics in such boundary regions is important in Section VIB. For the time being we note that as long as the classical superpotential of the theory lifts all flat directions, such boundary regions are not accessible and we do not need to worry about them.

Having understood the properties of the moduli space we turn on the tree level superpotential. The full superpotential of the theory with three vector-like flavors can be written as

$$
W_{3}=L\left(Y \cdot M^{2} \cdot X^{k-1}-\frac{N}{3} Z \operatorname{Pf} X-\Lambda^{2(2 N+1)}\right)+m_{a}^{i} M_{i}^{a}+\lambda^{i j} X_{i j},
$$

where $m_{a}^{i}$ is a rank three mass matrix, and the matrix of Yukawa couplings $\lambda$ is chosen so that all classical flat directions are lifted. We can now vary the masses $m_{a}^{i}$ to move in the 
parameter space between the $N_{f}=3$ and $N_{f}=0$ theories. However, it is useful to first choose only one mass eigenvalue to be large, so that the effective description is of two light vector-like flavors. In such a case the superpotential takes the form

$$
W_{2}=\frac{\Lambda_{(2)}^{4 N+3}}{\epsilon_{a c} Y^{a} M_{i_{1}}^{c} \epsilon^{i_{1} \ldots i_{2 N-1}} X_{i_{2} i_{3}} \cdot X_{i_{2 N-2} i_{2 N-1}}}+m_{a}^{i} M_{i}^{a}+\lambda^{i j} X_{i j}
$$

where the low energy scale $\Lambda_{(2)}$ is given by the usual scale matching condition, $\Lambda_{(2)}^{4 N+3}=$ $m \Lambda^{4 N+2}$ and the tree level terms only include fields of the $N_{f}=2$ model. We note that the nonperturbative term in Eq. (62) is generated by a one instanton term in the gauge theory. By solving the equations of motion for the mesons $M_{i}^{a}$ it is easy to see that the F-flatness conditions can not be satisfied. Together with the regularity of the Kähler potential in any finite region of the moduli space and the absence of classical flat directions, this implies supersymmetry breaking (Poppitz and Trivedi (1996)). We note that for small masses $m \ll \Lambda$ and couplings $\lambda \ll 1$ the theory is in a semiclassical regime and the low energy theory is calculable. As the masses are increased, control of the Kähler potential, and as a result, calculability, are lost, yet supersymmetry remains broken. For large masses, $m \gg \Lambda$, the effective description is given by the $N_{f}=0$ models, so that we have given an additional argument for supersymmetry breaking in these noncalculable theories.

\section{VIOLATIONS OF INDIRECT CRITERIA FOR DSB}

So far we have concentrated on models satisfying the Affleck-Dine-Seiberg (1985) criteria for dynamical SUSY breaking. These criteria restricted model building efforts to chiral models with $R$ symmetries and with no flat directions. In recent years a number of nonchiral models, models with classical flat directions, and models with no R-symmetry, have been shown to break supersymmetry dynamically. In this section we shall discuss such examples in turn.

\section{A. Non-chiral Models}




\section{SUSY QCD with Singlets}

We shall start the discussion of non-chiral models with SUSY QCD coupled to gauge singlet fields. We shall vary the number of flavors in the theory and analyze the quantum behavior along the classical flat directions. We should warn the reader that generically these models do not break supersymmetry. However, this analysis will lead us to the non-chiral ITIY model of DSB (Intriligator and Thomas, 1996a; Izawa and Yanagida, 1996) discussed in the following subsection. Along the way we shall develop useful techniques for the analysis of flat directions and illustrate them in additional examples in section VIB.

Consider an $S U(N)$ gauge theory with $N_{f}$ flavors coupled to a single gauge singlet field through the superpotential

$$
W=S Q_{i} \cdot \bar{Q}_{i}
$$

This superpotential lifts one classical flat direction of SUSY QCD, namely, $M_{i j}=v \delta_{i j}$. On the other hand, there is a flat direction along which $S$ is non-vanishing. Along this direction the vev of $S$ plays the role of a mass for the quark superfields 2 .

For large $S$ the effective theory is pure SYM with an effective strong coupling scale $\Lambda_{S Y M}^{3 N}=S^{N_{f}} \Lambda^{3 N-N_{f}}$, where $S$ denotes the expectation value, and $\Lambda$ is the original $S U(N)$ scale. Gaugino condensation in the effective theory generates the superpotential

$$
W=\Lambda_{S Y M}^{3}=S^{N_{f} / N} \Lambda^{3 N-N_{f}}
$$

The superpotential (64) gives an effective description at scales much smaller than $\langle S\rangle$, yet the fluctuations of $S$ itself remain massless, and (64) can be considered as an effective superpotential for this modulus, leading to the scalar potential

$$
V=\Lambda^{2 \frac{3 N-N_{f}}{N}}|S|^{2 \frac{N_{f}-N}{N}}
$$

\footnotetext{
${ }^{22}$ Note that unlike the case with a tree level mass term the superpotential preserves a nonanomalous R-symmetry, which is only broken spontaneously by the $S$ vev.
} 
Note that this effective description is only valid for $S \gg \Lambda$. We see that for $N_{f}<N$ this potential slopes to zero at infinity, and the vacuum energy is arbitrarily small for large $S$, exactly in the region where our effective description is reliable. For $N_{f} \geq N$ the potential for $S$ is non-vanishing at infinity (Affleck-Dine-Seiberg, 1985). Of course, the stabilization of this direction in the case $N_{f} \geq N_{c}$ does not imply supersymmetry breaking (or even the existence of a stable vacuum) in the full model. First, the analysis performed so far is not valid near the origin of field space. In addition there are many unlifted mesonic and baryonic flat directions. Yet, this suggests a way to stabilize other flat directions. Namely, one could couple the quarks to $N_{f}^{2}$ gauge singlet fields 23

$$
W=\sum_{i j}^{k} \lambda_{i j} S_{i j} Q_{i} \cdot \bar{Q}_{j},
$$

where the matrix of Yukawa coupling constants has maximal rank, and in the following we shall choose it to be $\lambda_{i j}=\lambda \delta_{i j}$. The superpotential (66) lifts all mesonic flat directions $M_{i j}=Q_{i} \bar{Q}_{j}$. If baryonic branches of the moduli space exist, they can be lifted by introducing additional nonrenormalizable couplings to singlets, but we shall leave these directions aside for the moment.

Along the singlet flat directions all quark superfields generically become massive, and the effective theory is pure SYM with the superpotential

$$
W=\lambda^{\frac{N_{f}}{N}} \tilde{S}^{\frac{N_{f}}{N}} \Lambda^{\frac{3 N-N_{f}}{N}}
$$

where $\tilde{S}=(\operatorname{det} S)^{\frac{1}{N_{f}}}$. This is just a direct generalization of Eq. (64), and we see that the flat direction is stabilized quantum mechanically for $N_{f} \geq N$. A somewhat more careful analysis

\footnotetext{
${ }^{23}$ In this case, the singlets transform under the global $S U\left(N_{f}\right)_{L} \times S U\left(N_{f}\right)_{R}$ group, and the chiral symmetry is preserved by the superpotential. It is spontaneously broken by the $S_{i j}$ vevs.

${ }^{24}$ Recall that the only fact we need to know about the Kähler potential to establish supersymmetry breaking is that it is non-singular in the appropriate variables. As a result one can further rescale $\lambda$ to one by field redefinitions, but it would be useful for us to keep it explicit.
} 
would show that the stabilization happens for all directions $S_{i j}$ as we shall see below.

To better understand the quantum behavior of the model, we shall repeat the above analysis in more detail. We write the scalar potential in the form 20

$$
V=\sum_{i}^{N_{f}}\left(\left|\frac{\partial W}{\partial Q_{i}}\right|^{2}+\left|\frac{\partial W}{\partial \bar{Q}_{i}}\right|^{2}\right)+\sum_{i j}^{N_{f}}\left|\frac{\partial W}{\partial S_{i j}}\right|^{2}
$$

where $W$ includes all possible nonperturbative contributions. A supersymmetric minimum in the model exists if all three terms in (68) vanish. The first two contributions in this potential reproduce the scalar potential of SUSY QCD with $N_{f}$ flavors and with the mass matrix $m_{i j}=\lambda S_{i j}$. We can, therefore use Eq. A24) of the Appendix to find the meson expectation values for which these terms vanish

$$
M_{i j}=\left(\operatorname{det}(\lambda S) \Lambda^{3 N-N_{f}}\right)^{1 / N}\left(\frac{1}{\lambda S}\right)_{i j} .
$$

Note that analyticity requires that (69) is satisfied for all values of $N_{f}$. We can now substitute this solution back into 68

$$
V=\sum_{i j}^{N_{f}}\left|\frac{\partial W}{\partial S_{i j}}\right|^{2}=|\lambda|^{2} \sum_{i j}\left|M_{i j}\right|^{2}=|\lambda|^{2 \frac{N_{f}}{N}}\left|\operatorname{det}(S) \Lambda^{3 N-N_{f}}\right|^{2 / N} \sum_{i j}\left|\left(\frac{1}{S}\right)_{i j}\right|^{2} .
$$

It is easy to see that this term is minimized by $S_{i j}=\tilde{S} \delta_{i j} \equiv(\operatorname{det} S)^{1 / N_{f}} \delta_{i j}$. Therefore the scalar potential for the lightest modulus $\tilde{S}$ is

$$
V=\left|\lambda^{N_{f}} \Lambda^{3 N-N_{f}} \tilde{S}^{N_{f}-N}\right|^{\frac{2}{N}}
$$

This is just the potential which could be derived from Eq. (67) and we again see that the flat direction is lifted if $N_{f} \geq N$. In fact now we can make a stronger statement. When $N_{f}=N+1$ the model is $s$-confining (Csaki-Schmaltz-Skiba, 1997d), and near the origin has a weakly coupled description in terms of composite (mesonic and baryonic) degrees of freedom. As a result the potential (70) is reliable near the origin, and we see that supersymmetry is restored there. When $N_{f}>N+1$ the weakly coupled description is given in terms of

\footnotetext{
${ }^{25}$ This potential, of course, is further modified by corrections to the Kähler potential.
} 
the dual gauge theory theory, and it is also possible to show that a supersymmetric vacuum exists at the origin.

The most interesting case for our purposes is $N_{f}=N$, where the vacuum energy is independent of the value of $\tilde{S}$ in the approximation that the Kähler potential is classical. This statement is equivalent to the statement that the energy is constant and non-vanishing everywhere on the mesonic branch of the moduli space. So far we have not considered the baryonic flat directions. In fact, in the model with $N_{f}=N$ flavors and the superpotential (66) the potential slopes to zero along the baryonic directions. However, it is easy to see that a simple modification leads to DSB (Intriligator and Thomas, 1996a; Izawa and Yanagida, 1996). This modification requires the introduction of two additional gauge singlet fields with nonrenormalizable couplings to the $S U(N)$ baryons (in the $N=2$ case the new couplings are renormalizable).

\section{The Intriligator-Thomas-Izawa-Yanagida Model}

Let us concentrate on a particular case with $S U(2)$ gauge group with two flavors of matter fields in the fundamental representation (four doublets $Q_{i}, i=1, \ldots, 4$ ). Because the matter fields are in the pseudoreal representation the superpotential (66) with $N_{f}^{2}$ singlets does not lift all the mesonic flat directions. ${ }^{2}$ Two mesonic flat directions remain and lead to a supersymmetric minimum at infinity in direct analogy with the baryonic flat directions for general $N$. A slight modification of the theory with $N_{f}^{2}+2=6$ singlets lifts all mesonic flat directions

$$
W=\sum_{i j} \lambda S_{i j} M_{i j}
$$

\footnotetext{
${ }^{26}$ Note that the superpotential (66) does not preserve the global $S U(4)_{F}$ symmetry of the $S U(2)$ with four doublets.
} 
where $M_{i j}=Q_{i} \cdot Q_{j}$, and $S_{i j}$ transform in the antisymmetric representation of the global $S U(4)_{F}$ symmetry. 7 Furthermore, we notice that near the origin of the moduli space, the theory has a weakly coupled description in terms of the mesons, $M_{i j}$. Thus our preceding discussion immediately leads us to the conclusion that supersymmetry is broken.

Let us understand qualitatively the mechanism of supersymmetry breaking. The nonperturbative dynamics generates the quantum constraint

$$
\operatorname{Pf}(M)=\Lambda_{2}^{4}
$$

This quantum constraint modifies the moduli space. While the origin $M_{i j}=0$ belongs to the classical moduli space, it does not lie on the quantum moduli space. On the other hand, the $S F$-terms only vanish at the origin, $M_{i j}=0$. Supersymmetry is therefore broken because the F-flatness conditions are incompatible with the quantum moduli space.

It is often convenient to impose the quantum constraint through a Lagrange multiplier in the superpotential. Then the full superpotential is

$$
W=\lambda S M+A\left(\operatorname{Pf} M-\Lambda_{2}^{4}\right)
$$

where $A$ is the Lagrange multiplier field. For $\lambda \ll 1$ the vacuum will lie close to the $S U(2)$ quantum moduli space. Thus one can consider the superpotential (72) as a small perturbation around the vacuum of the $N_{f}=N_{c}$ SQCD with masses $m=\lambda\langle S\rangle$. $\mho$ In this approximation the scalar potential is given again by (70) and is minimized when (up to symmetry transformations)

$$
\begin{gathered}
\tilde{S}=S_{12}=S_{34}, \\
S_{13}=S_{14}=S_{23}=S_{24}=0, \\
M_{12}=M_{34}=\frac{1}{\lambda \tilde{S}}\left(\frac{\Lambda_{2}^{4}}{\operatorname{Pf}(\lambda S)}\right)=\Lambda_{2}^{4} .
\end{gathered}
$$

\footnotetext{
${ }^{27}$ For simplicity we choose $\lambda$ to respect global $S U(4)$ symmetry, but this is not necessary.

${ }^{28}$ This approximation is equivalent to satisfying the equation of of motion for the Lagrange multiplier $A$.
} 
The vacuum energy is then given by

$$
V=|\lambda|^{2} \Lambda_{2}^{4}
$$

Thus, we have a non-chiral (left-right symmetric) model 29 which breaks supersymmetry! Indeed, as we mentioned in Section IIIA the Witten index can change discontinuously if the asymptotic behavior of the classical potential changes. Consider modifying the ITIY model by turning on a mass term for the singlet, $m S^{2}$. For sufficiently large mass, the low-energy effective theory is pure SYM, and the Witten index $\operatorname{Tr}(-1)^{F} \neq 0$. This is therefore true for any non-vanishing value of $m$. As the limit $m \rightarrow 0$ is taken, the asymptotic behavior of the potential changes (there is now a classical flat direction with $S \neq 0$ ) and the Witten index vanishes. Note that, in accord with our discussion of section IIIA, the potential $\sqrt{76}$ is flat along the $S$ flat direction.

At the level of analysis we performed so far, there is a pseudo-flat direction parameterized by $\tilde{S}$. Since $\tilde{S}$ is the only light field in the low energy theory and the superpotential (74) is exact, this direction would be exactly flat if the Kähler potential for $S$ were canonical. However, quantum contributions to the Kähler potential lift the degeneracy. For sufficiently small $\lambda$ and large $\lambda\langle S\rangle$ it is possible to show (Arkani-Hamed and Murayama, 1998; Dimopoulos et at, 1998) by renormalization group arguments that the quantum corrections due to the wave-function renormalization of $S$ are calculable and lead to a logarithmic growth of the potential at large $S$. It is possible to construct modifications of the ITIY models with calculable (but not necessarily global) supersymmetry breaking (Murayama, 1997; Dimopoulos et a, 1998). This is achieved by gauging a subgroup of the global symmetry under which $S$ transforms. As a result, the wave function renormalization of $S$ as well as the vacuum energy

\footnotetext{
${ }^{29}$ Practically, what is usually meant by a non-chiral model is that all fields can be given masses. This issue is a bit subtle in the ITIY model, as quark mass terms can be absorbed by a redefinition of the singlets. Still, one may first give masses to the singlets and integrate them out, and then introduce quark masses, so that ultimately all fields become heavy.
} 
depend on both the Yukawa and gauge coupling. For an appropriate choice of parameters, a local minimum of the potential exists for a large $S$ vev realizing Witten's (1981b) idea of inverted hierarchy in a model with dynamical supersymmetry breaking.

On the other hand, the exact superpotential of the theory (74) is of an O'Raifeartaigh type. Thus it is natural to ask whether there exists a region of the parameters of the model such that near the origin of the moduli space (where, in particular, the singlet vev is zero), the strong coupling dynamics decouples and the potential is calculable. Indeed, ChackoLuty-Ponton (1998) have argued that for sufficiently small coupling $\lambda$, and $\tilde{S} \ll \Lambda_{2} / \lambda$, the contributions of the strong dynamics to the scalar potential are small compared with the contributions of the light particles of mass $\lambda\langle S\rangle$. The latter contribution is calculable, and it was found in (Chacko-Luty-Ponton, 1998) that there exists a minimum of the potential at $\tilde{S}=0$. Moreover, it was argued that the calculability breaks down only when the Yukawa coupling $\lambda$ has non-perturbative strength. Finally, another minimum of the potential may exist at $\tilde{S} \sim \mathcal{O}\left(\Lambda_{2} / \lambda\right)$, however, this possibility can not be verified at present, since the strong coupling dynamics is important in this region.

We should also mention several obvious but useful generalizations of the ITIY model. Consider an $S P(N)$ gauge group with $N+1$ flavors of matter fields in the fundamental representation. This theory has an $S U(2 N+2)$ flavor symmetry. When the quarks are coupled to gauge singlet fields transforming in the antisymmetric representation of the flavor symmetry group, supersymmetry is broken in exactly the same way as in the $S U(2)$ model. A slightly more complicated generalization is based on an $S U(N)$ gauge group with $N_{f}=N$ flavors. In this case the baryonic operators $B$ and $\bar{B}$ are required to parameterize the quantum moduli space. Therefore, the superpotential (72) will not be sufficient for supersymmetry breaking. In particular there will be a supersymmetric solution $M_{i j}=0$, $B \bar{B}=\Lambda_{N}^{2 N}$. Supersymmetry is broken if two additional fields, $X$ and $\bar{X}$ with superpotential couplings $\lambda_{1} \bar{X} B+\lambda_{2} X \bar{B}$ are added to the superpotential. To enforce this structure of the superpotential one can gauge baryon number. We should note that in the case of the $S U(N)$ models, the renormalization group argument we used to show that the potential grows at 
large singlet vev's is not applicable to the $X$ and $\bar{X}$ directions. Other models with quantum modified moduli spaces can also break supersymmetry when each invariant appearing in the constraint is coupled to a gauge singlet Csaki-Schmaltz-Skiba (1997b).

\section{The 3-2 Model Revisited}

Before closing this section we would like to reanalyze, following Intriligator and Thomas (1996a), the familiar 3-2 model of Affleck-Dine-Seiberg discussed in section IVA in a different limit, $\Lambda_{2} \gg \Lambda_{3}$. We shall see that the description of supersymmetry breaking is quite different in this limit. First, note that from the point of view of the $S U(2)$ gauge group we have the matter content of the ITIY model, namely four $S U(2)$ doublets (three $Q$ 's and $L$ )

and six singlets $(\bar{u}$ and $\bar{d})$. The superpotential couplings of the 3-2 models are not sufficient to lift all classical flat directions, and in addition to the "singlets" there is an $S U(2)$ meson which can acquire a vev. (Of course all these flat directions, the including "singlet" ones, are lifted by $S U(3)$ D-terms as we learned in section IVA.) Let us parameterize the $S U(2)$ mesons by $M_{i j}=Q_{i} Q_{j}, M_{i 4}=Q_{i} L$, where the summation over $S U(2)$ indices is suppressed. In these variables the superpotential of the model is

$$
W=\mathcal{A}\left(\operatorname{Pf} M-\Lambda_{2}^{4}\right)+\lambda \bar{d}_{i} M_{i 4}
$$

where $\mathcal{A}$ is a Lagrange multiplier. Extremizing the superpotential with respect to $\bar{d}$ we find that the scalar potential contains terms

$$
V=\sum_{i}^{3}\left|M_{i 4}\right|^{2}+\ldots
$$

By an $S U(3)$ rotation we can set $M_{14}=M_{24}=0$. Thus supersymmetry is restored if it is possible that $M_{34}=\epsilon^{2} \rightarrow 0$. In turn this requirement and the quantum constraint (73) mean that

$$
M_{12}=\frac{\Lambda_{2}^{4}}{\epsilon^{2}} \rightarrow \infty
$$

At large expectation values the quantum moduli space approaches the classical one. Thus equation (79) can only be satisfied if the model possesses classical flat directions. But as we 
already know, when the $S U(3)$ D-flatness conditions are imposed, the model does not have flat directions. Therefore supersymmetry must be broken. The natural expectation values of the (canonically normalized) fields at the minimum of the potential are of order $\mathcal{O}\left(\Lambda_{2}\right)$, therefore the quantum corrections to the Kähler potential are significant and one can only estimate the vacuum energy in this limit, $V \sim\left|\lambda^{2} \Lambda^{4}\right|$.

\section{B. Quantum Removal of Flat Directions}

In the previous section we encountered the ITIY model which breaks supersymmetry even though it has a classical flat direction. Quantum mechanically, the potential becomes non-zero and flat (up to logarithmic corrections) far along this flat direction. It is in fact possible for quantum effects to completely "lift" classical flat directions, generating a growing potential along these directions. Thus it is possible for theories with classical flat directions to break supersymmetry, with a stable, supersymmetry-breaking minimum. We shall now build upon the insights gained in our analysis of of SQCD of the previous section, to develop a method for determining when classical flat directions are lifted quantum mechanically. We shall also discuss some examples in which this happens.

As will become clear from our discussion, a crucial requirement for the quantum removal of flat directions is that some gauge dynamics becomes strong along the flat direction. In many models, the opposite happens, that is, the gauge group is completely broken along the flat direction, and the dynamics becomes weaker as $S$ increases. However, it may be the case that along the flat direction, some gauge group remains unbroken, and fields charged under it obtain masses proportional to $S$. Then the dynamics associated with this gauge group becomes strong, and may lift the flat direction.

We should stress that in this section we shall be asking two separate questions. First, we shall ask if quantum effects can stabilize the potential along a given flat direction. It is most convenient to answer this question by finding a set of degrees of freedom which give a weakly

coupled description of the theory in the region of interest on the moduli space. However, if 
the quantum stabilization of the potential indeed happens, the vacuum may well lie in the genuinely strongly coupled region. Thus an affirmative answer to the first question is not sufficient to give an affirmative answer to our second question, whether supersymmetry is broken in the model. To answer this second question we shall need to consider the properties of the exact superpotential in the strong coupling region.

Following Shirman (1996), consider a model with classical flat directions. Assume for simplicity that there is a single modulus $S$. In the approximation of a canonical Kähler potential, the scalar potential of the model can be written as

$$
V=V_{r}+V_{S}=\sum\left|\frac{\partial W}{\partial \phi_{i}}\right|^{2}+\left|\frac{\partial W}{\partial S}\right|^{2}
$$

The applicability of this scalar potential is restricted by the assumption that the Kähler potential is canonical. However, for large enough $S$ vevs the description of the physics often simplifies, and in fact, it may be possible to find a description where the theory (or a sector of the theory) is weakly coupled. In such a limit, it is convenient to analyze the theory in two steps. First, one considers a "reduced" theory with the scalar potential $V_{r}$, where $S$ plays a role of the fixed parameter. One then studies the behavior of the scalar potential of the "reduced" theory as a function of $S$ as well as contributions of $V_{S}$. Let us consider various possibilities.

\section{A SUSY-breaking reduced theory}

Suppose that the potential $V_{r}$ in the reduced theory along the flat direction is non-zero, so that the reduced theory breaks supersymmetry. If $V_{r}$ is an increasing function of $S$, it is clear that the flat direction is stabilized. Typically, $V_{r} \rightarrow 0$ as $S \rightarrow 0$, but even if $V_{r}$ tends to a nonvanishing constant one can not conclude at this stage that supersymmetry is broken in the full theory. This is because the theory is typically in a strong coupling regime near the origin of the moduli space and therefore the assumption of a canonical Kähler potential as well as the separation of the scalar potential into the sum of two terms is not justified. An

example of a model with such behavior is an $S U(4) \times S U(3) \times U(1)$ model of Csaki-RandallSkiba (1996), see Section VIIA. Classically there is a flat direction along which the gauge 
group is broken down to $S U(4) \times U(1)$ and the matter is the same as in the $4-1$ model discussed in Section IVB. The strong coupling scale of the effective $S U(4)$ gauge group grows with the modulus, and the flat direction is stabilized (Shirman, 1996). Additional analysis performed by Csaki-Randall-Skiba (1996) shows that there is no supersymmetric vacuum at the origin, thus allowing them to conclude that SUSY is broken. This mechanism of quantum removal of classical flat directions is quite generic for discarded generator models (see Section VII A).

Another possibility is that $V_{r}$ is a decreasing function of the modulus leading to a runaway behavior at moderate values of $S$. In this case one should include in the analysis contributions from $V_{S}$. Since the stable vacuum (if it exists at all) will be found at large values of $S$, the separation of the scalar potential into two terms is well justified. We can therefore conclude that as long as $V_{S}$ stabilizes the flat direction, SUSY is broken. If, however, $V_{S} \rightarrow 0$ as $S \rightarrow \infty$ the theory does not have a stable vacuum. A very basic example of such behavior is the antisymmetric tensor models discussed in Section [IID4. In these models the effective theory along the classical flat direction is SUSY breaking $S U(5)$ with the scale vanishing at the boundary of the moduli space. The theory does not have a stable

vacuum. Introducing a tree level superpotential lifts all classical flat directions, stabilizes the potential and breaks supersymmetry. Note that there is no weak coupling description anywhere on the moduli space of the model. This means that the separation of the scalar potential into $V_{r}$ and $V_{S}$ is not apriori justified. However, $V_{r}$ arises from non-perturbative effects in the Kähler potential while $V_{S}$ arises from the tree level superpotential. As a result there is no interference effects between $V_{r}$ and $V_{S}$ and we can separate the potential into two positive definite terms.

\section{A Supersymmetric Reduced Theory}

Now we would like to consider models where $V_{r}=0$ has solutions for all values of $S$ (or for a set of moduli). In these cases we have to analyze the behavior of $V_{S}$ subject to the condition that $V_{r}=0$ is satisfied. It is instructive to consider as examples two classes of analogous 
models with the gauge groups $S P(N / 2) \times S U(N-1)$ (Intriligator and Thomas, 1996a) and $S U(N) \times S U(N-1)$ (Poppitz-Shadmi-Trivedi, 1996a).

We begin with the model of Intriligator and Thomas (1996a). The matter content is $Q \sim(N, N-1), L \sim(N, 1), R_{a} \sim(1, \overline{N-1})$, with the tree level superpotential:

$$
W_{\text {tree }}=\lambda Q L \bar{R}_{2}+\frac{1}{M} \sum_{a, b>2}^{N} \lambda_{a b} Q^{2} \bar{R}_{a} \bar{R}_{b} .
$$

This superpotential leaves classical flat directions associated with the $S U(N-1)$ antibaryons $\bar{b}^{a}=\left(\bar{Q}^{N-1}\right)^{a}=v^{N-1}$ (we shall denote the $R$ vevs by $v$ ). The exact superpotential was found in (Intriligator and Thomas, 1996a), and was used to show that there is no supersymmetric vacuum in the finite region of moduli space. Here we shall confine ourselves to discussing the physics along the classical baryonic flat directions.

Without loss of generality, we can consider the flat direction $S \equiv \bar{b}^{1}=v^{N-1}$. Along this direction, $S U(N-1)$ is completely broken. On the other hand, all $S P$ flavors get masses proportional to $v$ through the tree-level superpotential, so that one is left with a pure $S P(N / 2)$ which gets stronger for larger $S$. Gaugino condensation in this group then produces the superpotential

$$
W_{S} \sim S^{\frac{2}{N+2}}
$$

leading to the potential

$$
V_{S}=\left|\frac{\partial W}{\partial S}\right|^{2} \sim S^{-\frac{2 N}{N+2}}
$$

(Note that here $V_{r}=0$.) Actually, one can obtain this result starting from the exact superpotential. However, at scales $S \gg \Lambda_{1}$ the relevant degrees of freedom are the elementary ones, so we should consider the behavior of the potential in terms of $v$,

$$
V_{S} \sim v^{2 \frac{N-4}{N+2}}
$$

We see that for $N>4$ it increases along the classical flat direction. Thus the classical flat direction is stabilized quantum mechanically. The analysis of the theory in the finite region

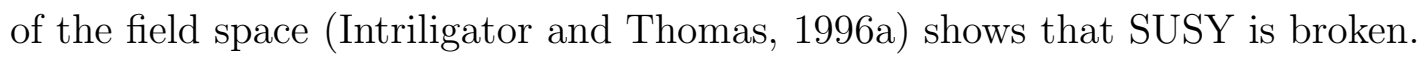


We would like to compare these results with the behavior of the model of Poppitz-ShadmiTrivedi (1996a) based on an $S U(N) \times S U(N-1)$ gauge group with matter in the fundamental representations: $Q \sim(N, N-1), \bar{L}_{i} \sim(\bar{N}, 1)$, and $\bar{R}_{a}(1, \overline{N-1})$, where $i=1 \ldots N-1$, and $a=1 \ldots N$. The tree level superpotential is given by:

$$
W_{\text {tree }}=\sum_{i a} \lambda_{i a} Q \bar{L}_{i} \bar{R}_{a}+\alpha_{a} \bar{b}^{a},
$$

where $\bar{b}^{a}=\left(\bar{R}^{N-1}\right)^{a}$ is an antibaryon of $S U(N-1)$. This superpotential lifts all flat directions as long as the coupling are chosen so that (Poppitz-Shadmi-Trivedi, 1996a) $\lambda_{i a}$ has maximal rank and

$$
\lambda_{i a} \alpha_{a} \neq 0 \text {. }
$$

Since we are interested in understanding the physics along the flat directions we shall set $\alpha_{a}=0$. Then there are classical flat directions parameterized by the $S U(N-1)$ antibaryons, in complete analogy with the $S U(N-1) \times S P(N / 2)$ model discussed above. Again, along the direction $S \equiv \bar{b}^{N}, S U(N-1)$ is broken, and all flavors of $S U(N)$ obtain mass. $S U(N)$ gaugino condensation generates the potential

$$
V_{S} \sim S^{-2 \frac{N-1}{N}}
$$

Again, this potential can also be obtained from the exact superpotential of the theory, which was obtained in (Poppitz-Shadmi-Trivedi, 1996a). In terms of the vev of the elementary field $\bar{R}$ we then have

$$
V_{S} \sim v^{\frac{-2}{N}}
$$

Unlike in the case of the Intriligator-Thomas model the runaway behavior persists and the model does not have a stable vacuum state. Turning on $\alpha_{a}$ according to (86), all flat directions are lifted. This, together with the analysis of (Poppitz-Shadmi-Trivedi, 1996a), which shows that there is no supersymmetric minimum in the finite region of moduli space, allows one to conclude that supersymmetry is broken. 
As we mentioned in the beginning of this subsection, the key ingredient in quantum lifting of flat directions is that the dynamics of some gauge group becomes strong along the flat direction. This in fact happens in both the $S U \times S P$ and the $S U \times S U$ examples we saw above, as, along the relevant flat direction, one group factor remains unbroken and fields charged under it obtain masses. However, the numerical factors are such that the potential grows along the flat direction in the first example, and slopes to zero in the second.

While general criteria for the determination of the quantum behavior along classical flat directions do not exist, we have illustrated several techniques which are useful for the analysis. We should also stress that we have concentrated on the simplest examples with a single modulus. In more general situations it is not sufficient to perform an analysis for each flat direction separately, assuming that other moduli are stabilized. One should do a complete analysis allowing all moduli to obtain independent vevs consistent with $D$ and F-flatness conditions. In particular one should study the moduli which do not appear in the tree level superpotential.

\section{Supersymmetry Breaking with No R-symmetry}

In section IIIB we discussed the relation between SUSY breaking and R-symmetries. We saw that theories with a spontaneously broken R-symmetry and no flat directions break SUSY. We also saw that if R-breaking terms are added to the superpotential, SUSY is typically restored. We emphasized that both these statements assume that the superpotential is generic, that is, all terms allowed by the symmetries appear.

In this section we shall encounter a theory with the most general renormalizable superpotential allowed by symmetries, which breaks supersymmetry even though it does not have an R-symmetry. Furthermore, unlike the theory of section VA, it does not possess an effective R-symmetry in the low-energy description. As we shall see, the reason supersymmetry is broken is that the dynamical superpotential is not generic.

The model we describe is an $S U(4) \times S U(3)$ gauge theory studied by (Poppitz-Shadmi- 
Trivedi, 1996a), which is the first in a series of $S U(N) \times S U(N-1)$ models that we already discussed from a different perspective in Section VIB. Here we only state some of the results. The matter content is $Q \sim(4,3), \bar{L}_{i} \sim(\overline{4}, 1)$, and $\bar{R}_{a} \sim(1, \overline{3})$ with $i=1 \ldots 3, a=1 \ldots 4$. One can add the classical superpotential

$$
W=\lambda \sum_{i=1}^{3} Q \cdot \bar{L}_{i} \cdot \bar{R}_{i}+\lambda^{\prime} Q \cdot \bar{L}_{1} \cdot \bar{R}_{4}+\alpha\left(\bar{R}^{3}\right)^{1}+\beta\left(\bar{R}^{3}\right)^{4},
$$

with appropriate contractions of the gauge indices (in particular, $\left(\bar{R}^{3}\right)^{a}$ stands for the $S U(3)$ "baryon" with the field $\bar{R}_{a}$ omitted). This superpotential does not preserve any R-symmetry. It is the most general renormalizable superpotential preserving an $S U(2)$ global symmetry that rotates $\bar{L}_{2}, \bar{L}_{3}$ together with $\bar{R}_{2}, \bar{R}_{3}$. In addition, it lifts all the classical flat directions of the model. If we add nonrenormalizable terms to this superpotential, (supersymmetric) minima will appear at Planckian field strength. These extra minima will not destabilize the non-supersymmetric minima we shall be discussing. 30

As was shown in (Poppitz-Shadmi-Trivedi, 1996a), the theory breaks supersymmetry. This can be established by carefully analyzing the low energy theory. In the limit that the $S U(3)$ dynamics is stronger, $S U(3)$ confines, giving a low energy theory in which $S U(4)$ has four flavors. After $S U(4)$ confines one has an O'Raifeartaigh-like theory, with the fields $Y_{i a}=Q \cdot \bar{L}_{i} \cdot \bar{R}_{a}, \bar{b}^{a}=\left(\bar{R}^{3}\right)^{a}, \bar{B}=Q^{3} L^{3}, P_{a}=Q^{3}\left(Q \cdot \bar{R}_{a}\right)$, and $B=\operatorname{det}(Q \cdot \bar{R})$ (the last two vanish classically). Taking into account the dynamically generated superpotential we find that the full superpotential is given by,

\footnotetext{
${ }^{30}$ Other models in this class (with $N>4$ ) are non-renormalizable, and we do not have a reason to neglect non-renormalizable operators. For $N \leq 6$ the generalization of (88) still gives the most general superpotential up to operators whose dimension is smaller or equal to the dimension of the baryon. Therefore, the expected SUSY breaking minimum is still a stable local minimum. In models with $N>6$, the most general superpotential with no R-symmetry and operators whose dimension does not exceed the dimension of the baryon operator will generically preserve supersymmetry.
} 


$$
W=\frac{P_{a} \bar{b}^{a}-B}{\Lambda_{3}^{5}}+A\left(P \cdot Y^{3}-\bar{B} B-\Lambda_{4}^{9} \Lambda_{3}^{5}\right)+\lambda \sum_{i=1}^{3} Y_{i i}+\lambda^{\prime} Y_{14}+\alpha \bar{b}^{1}+\beta \bar{b}^{4},
$$

where $A$ is a Lagrange multiplier, and $\Lambda_{4}, \Lambda_{3}$, are the scales of $S U(4), S U(3)$ respectively. This superpotential does not preserve any effective R-symmetry in terms of the variables of the low-energy theory. Still, as was shown in (Poppitz-Shadmi-Trivedi, 1996a), supersymmetry is broken. The crucial point is that the Lagrange multiplier $A$ only appears linearly in (89). If the superpotential contained terms with higher powers of $A$, supersymmetry would be restored. Note that the superpotential (89) is reminiscent of the superpotential (10) of the simplest O'Raifeartaigh model, with $A$ playing the role of $\phi_{1}$. In the absence of an R-symmetry, one cannot rule out the presence of higher powers of $\phi_{1}$ in (10), whereas in the dynamically generated superpotential (89), $A$ only appears linearly.

Other examples have been found which break supersymmetry even though the microscopic theory does not have an R-symmetry. These include, among others, the 4-31 model of (Leigh-Randall-Rattazzi, [1997), and the 4-1 model with the superpotential term $\operatorname{MPf} A$ (Poppitz and Trivedi, 1996), as well as, as we mentioned already, the ISS model (Intriligator-Seiberg-Shenker, 1995). In most of these examples, either the tree-level superpotential is not generic, or there is an effective R-symmetry in the low energy theory.

\section{DSB MODELS AND MODEL BUILDING TOOLS}

So far we have discussed several important models which illustrate the main methods and subtleties in the analysis of DSB. Many more models (in fact many infinite classes of models) have been constructed in recent years. The methods of analysis we have described can be used for these models. In fact often, not only the method of analysis but the dynamics itself is analogous to one or the other models discussed in previous sections. Thus it is not practical to present a detailed investigation of every known model of DSB.

On the other hand, in many cases the dynamics is not well understood beyond the conclusion that SUSY must be broken, and further investigation of the dynamics as well 
as the connection between different mechanisms and models of SUSY breaking may lead to better understanding of the general conditions for DSB. Therefore, in this section we shall give a list of known models briefly discussing how SUSY is broken. We shall emphasize the relations between various models, and give a partial classification. In addition we shall introduce a useful model building method which can be used to construct new models.

We shall also discuss in this section supersymmetry breaking in theories with anomalous $U(1)$ 's which give an example of dynamical SUSY breaking through the Fayet-Iliopolous mechanism.

\section{A. Discarded Generator Models}

Let us recall the observation that both the $4-1$ and the 3-2 models have a gauge group which is a subgroup of $S U(5)$, while the matter content (after adding $E^{+}$in the 3-2 model) falls into antisymmetric tensor and antifundamental representations - exactly as needed for DSB in $S U(5)$. Based on this observation, Dine et a (1996) proposed the following method of constructing new DSB models. Take a known model of dynamical supersymmetry breaking without classical flat directions and discard some of the group generators. This reduces the number of D-flatness conditions, and therefore, leads to the appearance of flat directions. On the other hand, the most general tree level superpotential allowed by the smaller symmetry may lift all the moduli. It is also possible that a unique non-perturbative superpotential will be allowed in such a "reduced" model. This construction is guaranteed to yield anomaly free chiral models which often possess a non-anomalous R-symmetry, and thus are good candidates for dynamical supersymmetry breaking. If a model constructed using this prescription breaks supersymmetry it is often calculable since for small superpotential couplings it typically possesses almost flat directions along which the effective description may be weakly coupled.

In fact, the 3-2 and 4-1 models are the simplest examples of two infinite classes of DSB

models based on $S U(2 N-1) \times S U(2) \times U(1)$ (Dine et a, [1996) and on $S U(2 N) \times U(1)$ 
(Dine et an, 1996: Poppitz and Trivedi, 1996) gauge groups which can be constructed by using the discarded generator method.

To construct these theories one starts with an $S U(2 N+1)$ theory with matter transforming as an antisymmetric tensor, $A$, and $2 N-3$ anti-fundamentals, $\bar{F}$. Then one requires gauge invariance under, for example, an $S U(2 N-1) \times S U(2) \times(1)$ subgroup, with the $U(1)$ generator being

$$
T=\operatorname{diag}(2, \ldots, 2,-(2 N-1),-(2 N-1)) .
$$

Under this group the matter fields decompose as

$$
\begin{aligned}
& A(\bar{\Xi}, 1,4), F(\square, 2,3-2 N), S(1,1,2-4 N), \\
& \bar{F}^{a}(\bar{\square}, 1,-2), \phi^{a}(1,2,2 N-1), \quad a=1, \ldots, 2 N-3 .
\end{aligned}
$$

The most general superpotential consistent with the symmetries is

$$
W=\gamma_{a b} A \bar{F}^{a} \bar{F}^{b}+\eta_{a b} S \phi^{a} \phi^{b}+\lambda_{a} F \bar{F}^{a} \phi^{a} .
$$

This superpotential lifts all classical flat directions. Models of this class have non-anomalous R-symmetry and supersymmetry is broken. It is interesting to observe that the coupling $\eta_{a b}$ in the superpotential above could be set to zero without restoring supersymmetry. While for $\eta=0$ classical flat directions appear, they are lifted by quantum effects.

The construction of the $S U(2 N) \times U(1)$ DSB series is quite analogous. The mater fields in this class of models are

$$
A_{2}, \quad F_{1-n}, \quad \bar{F}_{-1}^{a}, \quad S_{n}^{a}, \quad a=1, \ldots, 2 N-3,
$$

where subscripts denote $U(1)$ charges, and superscripts are flavor indices. With the most general superpotential allowed by symmetries the models break supersymmetry .

Clearly one can consider many other subgroups of $S U(2 N+1)$, and in fact, several other classes of broken generator models were constructed: $S U(2 N-2) \times S U(3) \times U(1)$ models (Csaki-Randall-Skiba, 1996; Chou, 1997), $S U(2 N-3) \times S U(4) \times U(1)$ and $S U(2 N-4) \times$ $S U(5) \times U(1)$ models (Csaki et a1, 1996). While these models are similar by construction 
to those we discussed above, the supersymmetry breaking dynamics is quite different, and various models in this class can have confinement, dual descriptions and quantum removal of classical flat directions. Since we have already considered the simplest and illuminating examples of these phenomena in DSB models, we shall not give a detailed discussion of all possible discarded generator models. We shall restrict ourselves to the mention of the $S P(2) \times U(1)$ model by Csaki-Schmaltz-Skiba (1997a). This model is interesting because it is an example of the discarded generator model in which the rank of the gauge group is reduced compared to the "parent" theory. The matter fields in this model are

\begin{tabular}{r|cr} 
& $S P(2)$ & $U(1)$ \\
\hline$A$ & $\boxminus$ & 2 \\
$Q_{1}$ & $\square$ & -3 \\
$Q_{2}$ & $\square$ & -1 \\
$S_{1}$ & 1 & 2 \\
$S_{2}$ & 1 & 4
\end{tabular}

Non-renormalizable couplings are required to lift all flat directions. The full superpotential is

$$
W=\frac{\Lambda^{7}\left(Q_{1} Q_{2}\right)}{2(A)^{2}\left(Q_{1} Q_{2}\right)^{2}-\left(Q_{1} A Q_{2}\right)^{2}}+Q_{1} Q_{2} S_{2}+Q_{1} A Q_{2} S_{1}
$$

where the first term is generated dynamically.

The existence of a general method for constructing discarded generator models suggests that there may exist a unified description of these models. In fact, Leigh-Randall-Rattazz (1997) found exactly such a description. It is based on the antisymmetric tensor models supplemented by a chiral field $\Sigma$ in the adjoint representation of the gauge group. We are interested in finding an effective description of the discarded generator models, or more generally of the models with $U(1)^{k-1} \times \prod_{i=1}^{k} S U\left(n_{i}\right)$ gauge groups (where $\sum_{i=1}^{k} n_{i}=2 N+1$ ) and the light matter given by decomposing the antisymmetric tensor and antifundamentals of $S U(2 N+1)$ under the unbroken gauge group. The adjoint $\Sigma$ needs to be heavy in such a vacuum. This can be achieved by introducing the superpotential for the adjoint 


$$
W_{\Sigma}=\sum_{i=2}^{k+1} \frac{s_{i}}{i} \operatorname{Tr} \Sigma^{i}
$$

We shall be most interested in the case $k=2$. For generic coefficients $s_{i}$ there are several discrete vacua where $\Sigma$ is heavy and the model contains matter in desired representations. Note that in the most symmetric vacuum $\Sigma=0$, the low energy physics is described by the antisymmetric tensor model, and SUSY is broken. For supersymmetry to be broken in other vacua one needs to lift the classical flat directions associated with the light fields which requires the following tree level superpotential

$$
W=\frac{1}{2} m \Sigma^{2}+\frac{1}{3} s_{3} \operatorname{Tr} \Sigma^{3}+\lambda_{1}^{i j} \bar{F}_{i} A \bar{F}_{j}+\lambda_{2}^{i j} \bar{F}_{i} A \Sigma \bar{F}_{j}+\lambda_{3}^{i j} \bar{F}_{i} \Sigma A \Sigma \bar{F}_{j}
$$

This superpotential is chosen so that in each vacuum of interest it exactly reproduces the superpotential needed for supersymmetry breaking. Leigh-Randall-Rattazzi (1997) showed that in the full model supersymmetry is broken for any value of the adjoint mass including $m=0$. This latter conclusion at first seems quite unusual, since the one loop beta function

coefficient of the model is $b_{0}=2 N+4$. In the $S U(2 N+1)$ model this might suggest that at least in the absence of the superpotential the theory is in a non-Abelian Coulomb phase. However, the analysis of Leigh-Randall-Rattazzi (1997) showed that indeed the superpotential is quite relevant and, when certain requirements on the Yukawa couplings are satisfied, SUSY is broken. A similar construction with $k>2$ (that is models with more than two non-Abelian factors and/or more than one abelian factor in the gauge group) was shown (Leigh-Randall-Rattazzi, 1997) not to break supersymmetry.

\section{B. Supersymmetry Breaking from an Anomalous $U(1)$}

Theories with an anomalous $U(1)$ provide a simple mechanism for supersymmetry breaking (Binetruy and Dudas, 1996; Dvali and Pomarol, 1996). Such theories contain a FayetIliopoulos (FI) term, so supersymmetry can be broken just as in the FI model we discussed in Section [IC2. In fact, the anomalous $U(1)$ theories discussed below are the only known 
examples where the Fayet-Iliopoulos mechanism of supersymmetry breaking can be realized dynamically. In the absence of any superpotential, at least one field with appropriate $U(1)$ charge develops a vev to cancel the FI term. One can then introduce an (effective) superpotential mass term for this field so that some $F$-term and the D-term cannot vanish simultaneously and supersymmetry is broken.

In our discussion of the FI model in Section IIC2 we simply put in a tree-level FI term by hand. It is well known that a $U(1)$ D-term can be renormalized at one loop (Fischler et a, 1981; Witten, 1981a). Such renormalization is proportional to the sum of the charges of the matter fields, and therefore, vanishes unless the theory is anomalous. Indeed, in many string models, the low-energy field theory contains an anomalous $U(1)$, whose anomalies are canceled by shifts of the dilaton-axion superfield, through the GreenSchwarz mechanism (Green and Schwarz, 1984). A FI term is generated for this $U(1)$ by string loops. As far as the low-energy field theory is concerned, we can treat this FI term as if it was put in by hand. The only subtleties associated with supersymmetry breaking involve the dilaton superfield.

Consider a theory with an anomalous $U(1)$ gauge symmetry with

$$
\delta_{G S}=\frac{1}{192 \pi^{2}} \sum_{i} q_{i}
$$

where $q_{i}$ denote the $U(1)$ charges of the different fields of the theory. The dilaton superfield $S$ then transforms as

$$
S \rightarrow S+i \frac{\delta_{G S}}{2} \alpha
$$

under the $U(1)$ transformation $A_{\mu} \rightarrow A_{\mu}+\partial_{\mu} \alpha$, where $A_{\mu}$ is the $U(1)$ vector boson. To be gauge invariant, the dilaton Kähler potential is then of the form

$$
K=K\left(S+S^{*}-\delta_{G S} V\right)
$$

where $V$ is the $U(1)$ vector superfield. This then gives the FI term

$$
\xi^{2}=-\frac{\delta_{G S}}{2} \frac{\partial K}{\partial S}
$$


Following (Arkani-Hamed-Dine-Martin, 1998) we shall consider the model of (Binetruy and Dudas, (1996), which has, in addition to the anomalous $U(1)$, an $S U(N)$ gauge symmetry. The model contains the field $\phi$, an $S U(N)$ singlet with $U(1)$ charge -1 (assuming $\delta_{G S}>0$ ), and one flavor of $S U(N)$, that is, fields $Q$ and $\bar{Q}$ transforming as $(N, q)$ and $(\bar{N}, \bar{q})$ under $S U(N) \times U(1)$. Working in terms of the $S U(N)$ meson $M=Q \bar{Q}$, the superpotential is given by

$$
W=m M\left(\frac{\phi}{M_{P}}\right)^{q+\bar{q}}+(N-1)\left(\frac{\Lambda^{3 N-1}}{M}\right)^{\frac{1}{N-1}}
$$

where the first term is a tree-level term, and the second term is generated dynamically by $S U(N)$ instantons. The potential will also contain contributions from the $U(1)$ D-term, which is given by,

$$
D=-g^{2}\left((q+\bar{q})|M|-|\phi|^{2}+\xi^{2}\right),
$$

where $g$ is the $U(1)$ gauge coupling. Minimizing the potential, one finds that supersymmetry is broken. $\phi$ wants to develop a vev to cancel $\xi^{2}$, but because of the $S U(N)$ dynamics, the meson $t$ develops a vev, which then generates, through the first term in (102), a mass term for $\phi$, so that the potential does not vanish.

It is important to recall though that the $S U(N)$ scale depends on the dilaton superfield. This dependence is most easily fixed by requiring that the second term in (102) is $U(1)$ invariant, giving,

$$
\Lambda^{3 N-1}=M_{P}^{3 N-1} e^{\frac{-2(q+\bar{q}) S}{\delta_{G S}}} .
$$

One can then minimize the potential in terms of $t, \phi$ and the dilaton, $S$. At the minimum, the D-term as well as the $t, \phi$, and dilaton $F$-terms are non-zero.

Note that if the dilaton superfield is neglected in the above analysis, the theory seems to have no Goldstino, as the gaugino and matter fermions obtain masses either by the Higgs mechanism or through the superpotential. In fact, as was shown in Arkani-Hamed-Dine Martin, 1998), the Goldstino in these theories is a combination of the gaugino, the matter fermion and the dilatino. In this basis the Goldstino wave-function is given by 


$$
\left(\frac{D}{\sqrt{2} g}, F_{i}, \sqrt{\frac{\partial^{2} K}{\partial S^{2}}} F_{S}\right),
$$

where $D, F_{i}$, and $F_{S}$ stand for the D-term, the $i$-th matter field $F$-term, and the dilaton $F$ term at the minimum, respectively.

\section{List of Models and Literature Guide}

Finally, in this section we shall present an extensive (but certainly incomplete) list of models known to break supersymmetry with references to the original papers where these models were introduced. While some of the models discussed below have been studied in great detail, frequently it is only known that a given model breaks supersymmetry, but the low energy spectrum and the properties of the vacuum have not been studied. In addition to the examples presented below many other models appeared in the literature. New supersymmetry breaking theories can be constructed from the known models in a variety of ways. Moreover, for phenomenological purposes it is often sufficient to find a model with a local non-supersymmetric minimum. While establishing the existence of a local non-supersymmetric minimum may sometimes be more difficult than establishing the absence of any supersymmetric vacuum, the methods involved in the analysis are essentially the same, and we shall not discuss such models here.

- $S U(5)$ with an antisymmetric tensor and an antifundamental Affleck-Dine-Seiberg, 1985; Meurice and Veneziand, 1984). The arguments of Affleck-Dine-Seiberg (1985) were based on the difficulty of satisfying 't Hooft anomaly matching conditions, while the Meurice and Veneziano (1984) argument was based on gaugino condensation. The model is not calculable. Murayama (1995) and Poppitz and Trivedil (1996) have used the method of

integrating in and out vector-like matter to give additional arguments for DSB in the model. Pouliot (1996) constructed a dual of the $S U(5)$ model and showed that the dual breaks SUSY at tree level. For the discussion of the model in the present review see Sections [IID and $\nabla \mathrm{Q}$. 
- $S U(2 N+1)$ with an antisymmetric tensor $A, 2 N-3$ antifundamentals $\bar{F}$ and the superpotential

$$
W=\lambda_{i j} A \bar{F}_{i} \bar{F}_{j},
$$

where $\lambda$ has the maximal rank (Affleck-Dine-Seiberg, 1985). These are generalizations of the SU (5) model. The integrating in and out method was used by Poppitz and Trivedi (1996) to further analyze these models. See Section IIID and also Section VIIA.

- $S O(10)$ with a single matter multiplet in the spinor representation (16 of $S O(10))$ (Affleck-Dine-Seiberg, 19840). The analysis of supersymmetry breaking in this model is very similar to that of the non-calculable $S U(5)$ model. Indeed, the $S U(5)$ model may be constructed from the $S O(10)$ model by using the discarded generator method. $\mathrm{Mu}-$ rayama (1995) discussed DSB in this model in the presence of an extra field in the vector 10 representation of $S O(10)$. For a small mass of the extra field, the theory is calculable, and assuming no phase transition, SUSY remains broken when the vector is integrated out. Pouliot and Strassler (1996) considered the same theory by adding an arbitrary number $N>5$ of vector fields and constructing the dual $S U(N-5)$ theory. They showed that the dual breaks SUSY when masses for the vectors are turned on. All these arguments can only be used as additional evidence of DSB in the $S O(10)$ model, but do not allow one to analyze the vacuum and low energy spectrum of the theory.

- The two generation SU(5) model (Meurice and Veneziano, 1984; Afflec-Dine-Seiberg, 1984d): $S U(5)$ with two antisymmetric tensors and two antifundamentals, with the superpotential

$$
W=\lambda A_{1} \bar{F}_{1} \bar{F}_{2} .
$$

Afflec-Dine-Seiberg (1984d) showed that the model is calculable. In fact, historically, this is the first calculable model with supersymmetry breaking driven by an instanton-induced superpotential. The vacuum and the low energy spectrum of the model were analyzed in detail by ter Veldhuis (1996). ter Veldhuis (1998) also analyzed generalizations of this model which include extra vector-like matter with a mass term in the superpotential. 
- The 3 - 2 model (Affleck-Dine-Seiberg, 1985): $S U(3) \times S U(2)$ with

$$
Q(3,2), \quad \bar{u}(\overline{3}, 1), \quad \bar{d}(\overline{3}, 1), \quad L(1,2),
$$

with the superpotential

$$
W=\lambda Q L \bar{d} .
$$

This model is calculable. The analysis of the vacuum and low energy spectrum can be found in (Affleck-Dine-Seiberg, 1985; Bagger-Poppitz-Randall, 1994). The model possesses a global $U(1)$ symmetry which can be gauged without restoring SUSY, the relevant details of the vacuum structure in this case can be found in (Dine-Nelson-Shirman, 1995). See Sections IVA and VIA2.

- Discarded generator models: These include $S U\left(n_{1}\right) \times S U\left(n_{2}\right) \times U(1)$ and $S U(2 N) \times(1)$ subgroup of $S U(2 N+1)$ (with $n_{1}+n_{2}=2 N+1$ ) with matter given by the decomposition of the antisymmetric tensor and $2 N-3$ antifundamentals of $S U(2 N+1)$ under the appropriate gauge group. This construction was proposed in (Dine et a a, 1996). For details see Section VIIA. The two smallest models in this class are the $3-2$ model (Section $\mathbb{V A}$ ) and the 4-1 model (Section IVB). The $S U(2 N) \times U(1)$ models were first constructed in (Ding et a1, 1996; Poppitz and Trived, 1996); the $S U(2 N-1) \times S U(2) \times U(1)$ models can be found in (Dine et ad, 1996); the $S U(2 N-2) \times S U(3) \times U(1)$ models are considered in 8 (Csaki-Randall-Skiba, 1996); finally, the $S U(2 N-3) \times S U(4) \times U(1)$ models are discussed in (Csaki et a , 1996). A unified description of this class of models, as well as of the noncalculable $S U(2 N+1)$ models, is given in (Leigh-Randall-Rattazzi, 1997). Another example of the models in this class is $S P(2) \times U(1)$ model of Csaki-Schmaltz-Skiba (1997a).

- $S U(2 N+1) \times S U(2)($ Dine et ad, 1996) with

$$
Q \sim(\square, \square), \quad L \sim(1, \square), \quad Q_{i} \sim(\bar{\square}, 1), \quad i=1,2,
$$

\footnotetext{
${ }^{31}$ See also (Chou, 1997).
} 
and with a superpotential similar to that of the $3-2$ model. These models are obvious generalizations of the $3-2$ model. The dynamics in this class of models is very similar to that of the 3-2 model. For detailed analysis see (Intriligator and Thomas, 1996b). The low energy physics of the $S U(5) \times S U(2)$ model in this class, in the limit of a strong $S U(2)$, is described by the non-calculable $S U(5)$ model (Intriligator and Thomas, 1996b) to which we paid so much attention in this review.

- $S U(7) \times S P(1)$ and $S U(9) \times S P(2)$ (โntriligator and Thomas, 1996b): These models are obtained by dualizing the $S U(7) \times S U(2)$ and $S U(9) \times S U(2)$ models of the previous paragraph. The matter content is

$$
\begin{array}{lll}
A(\boxminus, 1), & F(\square, 1), & \bar{P}(\bar{\square}, \square), \\
L(1, \square), & \bar{U}(\bar{\square}, 1), & \bar{D}(\bar{\square}, 1),
\end{array}
$$

and the superpotential

$$
W=A \overline{P P}+F \bar{P} L
$$

Note that these models can be constructed starting from the antisymmetric tensor models of Affleck-Dine-Seiberg (1985), by gauging a maximal global symmetry and adding matter to cancel all anomalies with the most general superpotential.

- $S U(2 N+1) \times S U(2)$ with

$$
A \sim(A, 1), \quad F \sim(\square, 2), \quad \bar{F}_{i} \sim(\bar{\square}, 1), \quad D \sim(1,2),
$$

where $i=1, \ldots, N-2$. (Dine et at, 1996) To lift all flat directions, a non-renormalizable superpotential is required

$$
W=\sum_{i, j=1}^{2 N-2} \gamma_{i j} A \bar{F}_{i} \bar{F}_{j}+\lambda \bar{F}_{2 N-1} F D+\frac{1}{M} \sum_{i, j=1}^{2 N-2} \alpha_{i j} \bar{F}_{i} \bar{F}_{j} F F
$$

- $S U(2 N+1) \times S P(M), N \geq M-1$ with

$$
Q \sim(\square, \square), \quad \bar{Q}_{i} \sim(\bar{\square}, 1), \quad L \sim(1, \square)
$$


where $i=1, \ldots, 2 M$ is a flavor index (Dine et $a$, 1996). These are generalization of the 3-2 model with a non-renormalizable superpotential.

The $S U(2 N+1)$ dynamics generates a dynamical superpotential (Dine et a, 1996). In addition, a quantum constraint is generated by the $S P(M)$ dynamics for $N=M$. The tree level superpotential

$$
W=\lambda \bar{Q}_{2} Q L+\sum_{i, j>2}^{2 M} \gamma_{i j} Q^{2} \bar{Q}_{j} \bar{Q}_{j}
$$

lifts all flat directions, and supersymmetry is broken. For details see (Dine et a4, 1996; Intriligator and Thomas, 1996a).

For $N=M+1$, the tree level superpotential (116) does not lift all classical flat directions, yet they are lifted by nonperturbative effects (Intriligator and Thomas, 1996a; Shirman, 1996) and SUSY is broken. We discussed this model in Section VIB. (Note that in that section, we used a different notation for $S P$ and refered to this theory as $S U(N-1) \times$ $S P(N / 2)$.)

It is also useful to note that for $M+1<N$, the $S P(M)$ dynamics can have dual description. Intriligator and Thomas (1996b) argued that the dual description (with $S U(2 N+1) \times S P(N-M-1)$ gauge group and matter content which includes the symmetric tensor of $S U(2 N+1)$ as well as (anti)fundamentals and bifundamentals breaks SUSY. When $N>3 M+2$ it is only the dual description which is asymptotically free and can be interpreted as a microscopic theory.

An interesting modification of these models (Luty and Terning, 1998) is an $S U(2 N+$ 1) $\times S P(N+1)$ theory with

$$
Q \sim(\square, \square), \quad \bar{Q}_{i} \sim(\bar{\square}, 1), \quad L_{a} \sim(1, \square)
$$

where $i=1, \ldots, 2(N+1)$ and $a=1, \ldots, 2 N+1$. We note that this version of the model possesses an $S U(2 N+1) \times U(1) \times U(1)_{R}$ global symmetry. Luty and Terning (1998) considered only the renormalizable superpotential

$$
W=\lambda \bar{Q} Q L
$$


where $\lambda$ has maximal rank. They showed that while this model has a large number of classical flat directions, all of them are lifted quantum mechanically. One can now add mass terms for some flavors of the $S P(N+1)$ fields $L_{a}$. For an appropriately chosen mass matrix supersymmetry remains broken. Choosing a mass matrix of maximal rank and integrating out the massive matter, we recover the non-renormalizable model discussed above.

- Non-renormalizable $S U(2 N) \times U(1)$ model (Dine et ad, 1996) with chiral superfields transforming under the gauge group and a global $S U(2 N-4)$ symmetry as

$$
A \sim(\boxminus, 2 N-4,1), \quad \bar{F} \sim(\bar{\square},-(2 N-2), \bar{\square}), \quad S \sim(1,2 N, \square) .
$$

The superpotential required to stabilize all flat directions

$$
W=A \overline{F F} S
$$

explicitly breaks the global symmetry down to a subgroup. The anomaly free $S U(N-2)$ subgroup of the global symmetry can be gauged without restoring SUSY.

- Non-renormalizable $S U(N) \times U(1)$ models (Dine et at, 1996): The matter content is (we also give charges under a maximal global $S U(N-3)$ symmetry)

$$
\begin{aligned}
A \sim(\boxminus, 2-N, 1), \quad & \quad \sim(M, 1,1), \quad \bar{N}_{i} \sim(\bar{\square}, N-1, \bar{\square}), \\
S_{i} & \sim(1,-N, \square)), \quad S_{i j} \sim(1,-N, \boxminus),
\end{aligned}
$$

where $i, j=1, \ldots, N-3$. The superpotential

$$
W=\lambda_{i} \bar{N}_{i} N S_{i}+\gamma_{i j} A \bar{N}_{i} \bar{N}_{j} S_{i j}
$$

lifts all flat directions while preserving a global symmetry. Note that for $N=4, S_{i j}$ does not exist and this is just the $4-1$ model of Section IVB.

- $S U(N) \times S U(N-1)$ (Poppitz-Shadmi-Trivedi, 1996a), and $S U(N) \times S U(N-$ 2) (Poppitz-Shadmi-Trived, 1996b): The $S U(N) \times S U(N-1)$ models were discussed in Section VIB]. We discussed SUSY breaking without R-symmetry in these models in Section $\mathrm{VIC}$. The $S U(N) \times S U(N-2)$ models, which are similar by construction but have very 
different dynamics are discussed in Section $\nabla \mathrm{B}$. Both classes of models have calculable minima with an unbroken global symmetry $(S U(N-2)$ and $S P(N-3)$ respectively) (Poppitz and Trivedi, 1997; Arkani-Hamed et al, 1998; Shadmi, 1997).

- ITIY models (Intriligator and Thomas, 1996a; Lzawa and Yanagida, 1996) and their modifications: We discussed the ITIY models in section VIA2. They are based on an $S U(N)$ $(S P(N))$ gauge group with $N(N+1)$ flavors of matter in the fundamental representation coupled to a set of gauge singlet fields in such a way that all D-flat directions are lifted. Even after supersymmetry breaking these models posses a flat direction which is only lifted by (perturbative) corrections to the Kähler potential. In (Shirman, 1996; Arkani-Hamed and Murayama, 1998; Dimopoulos et a4, 1998) it was argued that the perturbative corrections generate a growing potential for large vevs along this direction. By gauging a subgroup of the global symmetry it is possible to obtain a modification of the model with a calculable local SUSY breaking minimum at large vevs (Murayama, 1997; Dimopoulos et a, 1998). These models can be generalized in the following way. Take any model with a quantum modified constraint and couple all gauge invariant operators to singlet fields. Since the quantum constraint becomes incompatible with the singlet F-term conditions, supersymmetry must be broken (Csaki-Schmaltz-Skiba, 1997D). As an example consider an $S O(7)$ gauge group with five matter multiplets transforming in the spinor representation. The theory possesses an $S U(5)$ global symmetry, and a $U(1)_{R}$ under which all matter fields are neutral. The gauge invariant composites transform as an antisymmetric tensor $A$ and antifundamental $\bar{F}$ of the global symmetry. The quantum constraint is

$$
A^{5}+A \bar{F}^{4}=\Lambda^{10} .
$$

Coupling all gauge invariants to gauge singlets $\tilde{A}$ and $F$ and implementing the constraint through the Lagrange multiplier $\lambda$ one finds

$$
W=\tilde{A} A+F \bar{F}+\lambda\left(A^{5}+A \bar{F}^{4}-\Lambda^{10}\right),
$$

and obviously SUSY is broken. We note that the model is nonrenormalizable. 
- $S U(2)$ with one $I=3 / 2$ matter field (Intriligator-Seiberg-Shenker, 1995): We discussed this theory in section $\nabla \mathrm{A}$. A non-renormalizable tree-level superpotential lifts all classical flat directions. The theory confines, no superpotential is generated dynamically, and supersymmetry is broken since the tree-level superpotential cannot be extremized in terms of the confined field.

- $S U(7)$ with two symmetric tensors, $S_{a}, a=1,2$, six antifundamentals $\bar{Q}_{i}, i=1, \ldots, 6$, and the tree level superpotential

$$
W=\sum_{i}^{3} S^{1} \bar{Q}_{2 i} \bar{Q}_{2 i-1}+S^{2} \bar{Q}_{2 i} \bar{Q}_{2 i+1}
$$

where in summing over $i$ we identify $7 \sim 1$ Csaki-Schmaltz-Skiba, 1997b; Nelson and Thomas, 1996). This is another example of SUSY breaking through confinement, which we saw in the Intriligator-Seiberg-Shenker (1995) model in Section VA. The superpotential lifts all classical flat directions while preserving a global anomaly free $U(1) \times U(1)_{R}$ symmetry. $S U(7)$ dynamics leads to confinement, and generates the nonperturbative superpotential

$$
W_{d y n}=\frac{1}{\Lambda^{13}} H^{2} N^{2}
$$

where $H_{i j}^{a}=S^{a} \bar{Q}_{i} \bar{Q}_{j}$ and $N_{i}=S^{4} \bar{Q}_{i}$. Near the origin of the moduli space the Kähler potential is canonical in terms of the composite fields. Solving the equations of motion for $H_{i j}^{a}$ one finds that at least some of the composite fields acquire vevs, breaking the global symmetry, and therefore, supersymmetry.

- $S O(12) \times U(1)$ and $S U(6) \times U(1)$ (Csaki-Schmaltz-Skiba, 1997b). The matter content of the $S O(12) \times U(1)$ model is $(32,1),(12,-4), 1,8),(1,2),(1,6)$. The matter content of the $S U(6) \times U(1)$ model is $(20,1),(6,-3),(\overline{6},-3),(1,4),(1,2)$. These models are constructed by starting with a non-chiral theory with a dynamical superpotential and gauging a global $U(1)$ symmetry (adding the necessary fields to make the full theory anomaly free) in a way which makes the theory chiral. Supersymmetry is broken by the interplay between a dynamically-generated superpotential and the tree-level superpotential. 


\section{ACKNOWLEDGEMENTS}

It is a pleasure to thank Michael Dine, Galit Eyal, Jonathan Feng, Martin Gremm, and Yossi Nir for useful comments. Y. Shirman is grateful to the Aspen Center for Physics for hospitality during the early stages of this work. The work of Y. Shirman was supported in part by NSF grant PHY-9802484. The work of Y. Shadmi was supported in part by DOE grants \#DF-FC02-94ER40818 and \#DE-FC02-91ER40671, and by the Koret Foundation.

\section{APPENDIX A: SOME RESULTS ON SUSY GAUGE THEORIES}

In this appendix we shall briefly review some results in supersymmetric gauge theories. Our main goal here is to introduce notations (which will mainly follow those of (Baggen and Wess, 1991)), and to summarize the results necessary to make the present review self-contained. Much more detailed reviews of the progress in our understanding of supersymmetric gauge theories exist in the literature, e.g. (Intriligator and Seiberg, 1996; Peskin, 1997; Shifman, 1997).

\section{Notations and Superspace Lagrangian}

We shall consider an effective low-energy theory of light degrees of freedom well above the possible scale of supersymmetry breaking. In this case the effective action will have linearly realized supersymmetry, and it is convenient to write an effective supersymmetric Lagrangian in $\mathcal{N}=1$ superspace, where four (bosonic) space-time coordinates are supplemented by four anti-commuting (fermionic) coordinates $\theta_{\alpha}$, and $\bar{\theta}^{\dot{\alpha}}, \alpha=1,2$. The light matter fields combine into chiral superfields 32

$$
\Phi=\phi+\sqrt{2} \theta \psi+\Theta^{2} F,
$$

\footnotetext{
32 We shall usually use the same notation for a chiral superfield and its lowest scalar component.
} 
while gauge bosons and their superpartners combine into vector superfields

$$
V=-\theta \sigma^{\mu} \bar{\theta} A_{\mu}+i \theta^{2} \bar{\theta} \bar{\lambda}-i \bar{\theta}^{2} \theta \lambda+\frac{1}{2} \theta^{2} \bar{\theta}^{2} D
$$

where we have used Wess-Zumino gauge.

The effective supersymmetric Lagrangian for a theory with gauge group $G$, and matter fields $\Phi_{i}$ transforming in the representation $r$ of the gauge group (with $T^{a}$ being a generator of this representation) can be written as

$$
\mathcal{L}=\int d^{4} \theta K\left(\Phi^{\dagger}, e^{V \cdot T} \Phi\right)+\frac{1}{g^{2}} \int d^{2} \theta \mathcal{W}^{\alpha} \mathcal{W}_{\alpha}+\text { h.c }+\int d^{2} \theta W(\Phi)+\text { h.c. }
$$

The first term in (A3) is a Kähler potential which contains, among others, kinetic terms for the matter fields. The Kähler potential also contributes gauge-interaction terms to the scalar potential. The second term in (A3) is the kinetic term for the gauge fields. In particular $\mathcal{W}^{\alpha}=-\frac{1}{4} \overline{\mathcal{D} \mathcal{D}} \mathcal{D}_{\alpha} V$, where $\mathcal{D}$ is a superspace derivative, is a supersymmetric generalization of the gauge field-strength $F^{\mu \nu}$. The last term in (A3) is the superpotential.

The superpotential is a holomorphic function of chiral superfields and obeys powerful non-renormalization theorems. In particular, in perturbation theory the superpotential can only be modified by field rescalings (which can be absorbed into renormalization of the Kähler potential). Using holomorphy, symmetries of the theory, and known weakly coupled limits it is often possible to determine the superpotential exactly, including all nonperturbative effects. Similarly, the kinetic term for the gauge multiplet is a holomorphic function allowing one to obtain exact results on the renormalization of the gauge coupling.

The Kähler potential on the other hand can be a general real-valued function of $\Phi^{\dagger}$ and $\Phi$ consistent with symmetries. Classically it is given by

$$
K=\Phi^{\dagger} e^{V \cdot T} \Phi,
$$

but quantum mechanically it is renormalized both perturbatively and non-perturbatively.

In studying the dynamical behavior of the supersymmetric theory it is often useful to remember that the Hamiltonian is determined by the supersymmetry generators 


$$
H=\frac{1}{4}\left(\bar{Q}_{1} Q_{1}+Q_{1} \bar{Q}_{1}+\bar{Q}_{2} Q_{2}+Q_{2} \bar{Q}_{2}\right)
$$

¿From Eq. (A5) we see that a supersymmetric vacuum state (a state annihilated by the supersymmetry charges) has vanishing energy. Therefore, a particularly important role (especially in the analysis of supersymmetry breaking) is played by the scalar potential of the theory

$$
V=\frac{1}{2} g^{2} \sum_{a}\left(D^{a}\right)^{2}+F_{i}^{\dagger} g_{i j}^{-1} F_{j},
$$

where $g_{i j}=\frac{\partial^{2} K}{\partial \Phi^{\dagger} \partial \Phi^{j}}$, and the auxiliary fields $F$, and $D$ are given in terms of the scalar fields by

$$
\begin{aligned}
& F_{i}=\frac{\partial}{\partial \Phi_{i}} W \\
& D^{a}=\sum_{i} \Phi^{\dagger i} t^{a} \Phi^{i},
\end{aligned}
$$

where in $F_{i}$ one takes the derivatives of the superpotential with respect to the different superfields and then keeps only the lowest component, and in $D^{a}, \Phi^{i}$ stands for the scalar field of the $\Phi^{i}$ supermultiplet.

Typically a supersymmetric gauge theory possesses a set of directions in field space (called D-flat directions) along which $D^{a}=0$ for all $a$. Along some or all of these D-flat directions, the $F$-flatness conditions, $F_{i}=0$, can also be satisfied. The subspace of field space where the scalar potential vanishes is called a moduli space and to a large degree determines the low-energy dynamics.

The study of non-perturbative effects in SUSY gauge theories relies heavily on the use of symmetries. An important role, especially in the applications to dynamical supersymmetry breaking, is played by an "R-symmetry". We therefore pause to introduce this symmetry. Under an R-symmetry, the fermionic coordinates rotate as,

$$
\theta \rightarrow e^{i \alpha} \theta
$$

A chiral field with R-charge $q$ transforms under this symmetry as follows

$$
\Phi(x, \theta, \bar{\theta}) \rightarrow e^{-i q \alpha} \Phi\left(x, e^{i \alpha} \theta, e^{-i q \alpha} \bar{\theta}\right)
$$


Note that different component fields transform differently under R-symmetry, and thus it does not commute with supersymmetry. On the other hand, the vector superfield $V$ is neutral under R-symmetry (therefore, the gaugino transforms as $\lambda \rightarrow e^{-i \alpha} \lambda$ ). Clearly there always exists an assignment of R-symmetry charges to the superfields such that the Kähler potential contribution to the action is invariant under R-symmetry. On the other hand the superpotential contributions to the action explicitly break R-symmetry unless the superpotential has charge 2 under R-symmetry.

In the following subsections we shall discuss methods for determining the classical moduli space. We shall also describe the quantum behavior of supersymmetric QCD (SQCD) with various choices of the matter content. At the end of this section we shall comment on analogous result for models with different gauge groups and matter content. These results will provide us with tools needed to analyze supersymmetry breaking.

\section{D-flat Directions}

Classically, one could set all superpotential couplings to zero. Then the moduli space of the theory is determined by D-flatness conditions. Even when tree level superpotential couplings are turned on but remain small, the vacuum states of the theory will lie near the solutions of D-flatness conditions (still in the classical approximation). It is convenient, therefore, to analyze SUSY gauge theories in two stages. First find a submanifold in the field space on which the D-terms vanish, and then analyze the full theory including both tree level and non-perturbative contributions to the superpotential.

We start by describing a useful technique for finding the D-flat directions of a theory (Affleck-Dine-Seiberg, 1984d; Affleck-Dine-Seiberg, 1985) with $S U(N)$ gauge symmetry. Consider the $N \times N$ matrix

$$
D_{j}^{i}=\phi^{\dagger l}\left(A_{j}^{i}\right)_{l}^{k} \phi_{k}
$$

where $\left(A_{j}^{i}\right)_{l}^{k}$ are the real generators of $G L(N)$. For $\phi$ in the fundamental representation $\left(A_{j}^{i}\right)_{l}^{k}=\delta_{l}^{i} \delta_{j}^{k}$ (the generalization of $\left(A_{j}^{i}\right)$ for a general multi-index representation is obvious). 
It is easy to see that the vanishing of all $D^{a}$ 's is equivalent to the requirement that $D_{j}^{i}$ be proportional to the unit matrix, $D_{j}^{i} \sim \delta_{j}^{i}$. To show this it is sufficient to note that $D^{a}=D_{j}^{i} \lambda_{i}^{a j}$, where $\lambda^{a}$ are generators of $S U(N)$ in the fundamental representation.

Another way to parameterize the moduli space is by the use of gauge invariant composite operators. It has been shown that a complete set of such operators is in one to one correspondence with the space of D-flat directions (Luty and Taylor, 1996). An important feature of this latter parameterization of the moduli space is that in some cases there exist gauge invariant operators which vanish identically due to the Bose statistic of the superfields. For this reason they do not have counterparts in the "elementary" parameterization of the moduli space. However, due to quantum effects these operators typically describe light (composite) degrees of freedom of the low-energy theory and play an important role in the dynamics.

\section{Pure Supersymmetric $S U\left(N_{c}\right)$ Theory}

The Lagrangian of a pure supersymmetric Yang-Mills (SYM) theory can be written as

$$
\mathcal{L}=\frac{1}{4 g_{W}^{2}} \int d^{2} \theta \mathcal{W}^{\alpha} \mathcal{W}_{\alpha}+\text { h. c. . }
$$

The Wilsonian coupling constant in the Lagrangian can be promoted to a vev of the back-

ground chiral superfield $\frac{1}{g_{W}^{2}} \rightarrow S=\frac{1}{g_{W}^{2}}-i \frac{\Theta}{8 \pi^{2}}$. Since the physics is independent of shifts in $\Theta$, the Wilsonian gauge coupling in Eq. (A11) receives corrections only at one loop. On the other hand, the gauge coupling constant in the 1PI action receives contributions at all orders in perturbation theory. These two coupling constants can be related by field redefinitions (Shifman and Vainshtein, 1986; Shifman and Vainshtein, 1991). In the following we shall always use the Wilsonian action and work with Wilsonian coupling constants. We shall use functional knowledge of the exact beta functions only to establish the scaling dimensions of the composite operators in our discussion of duality in Section A7.

SYM is a strongly interacting non-abelian theory very much like QCD. In particular it 
is believed that it confines and develops a mass gap. By using symmetry arguments it is possible to show that if the gaugino condensate develops it has the form

$$
\langle\lambda \lambda\rangle=\text { const } \times \Lambda_{S Y M}^{3}=\text { const } \times \mu^{3} e^{-\frac{8 \pi^{2}}{N_{c} g^{2}}} .
$$

In fact the constant can be exactly calculated (Novikov et a, 1983; Shifman and Vainshtein, 1988). The theory has $N_{c}$ supersymmetric vacuum states.

\section{4. $N_{f}<N_{c}$ : Affleck-Dine-Seiberg Superpotential}

As a next step one can consider an $S U\left(N_{c}\right)$ gauge theory with $N_{f}\left(<N_{c}\right)$ flavors of matter fields in the fundamental $Q$ and antifundamental $\bar{Q}$ representations. This theory possesses a large non-anomalous global symmetry under which matter fields transform as follows:

$$
\begin{array}{ccccc} 
& S U\left(N_{f}\right)_{L} \times S U\left(N_{f}\right)_{R} \times & U(1)_{B} & \times & U(1)_{R} \\
Q & N_{f} & 1 & 1 & \frac{N_{f}-N_{c}}{N_{f}} \\
\bar{Q} & 1 & \bar{N}_{f} & -1 & \frac{N_{f}-N_{c}}{N_{f}}
\end{array}
$$

Classically there are D-flat directions along which the scalar potential vanishes. Using the techniques described above we can parametrize these flat directions (up to symmetry transformations) by

$$
Q=\left(\begin{array}{cccc}
v_{1} & & & \\
& v_{2} & & \\
& & \ldots & \\
& & & v_{N_{f}} \\
& & & \ldots \\
\ldots & \ldots & \ldots & \ldots
\end{array}\right)=\bar{Q}
$$

These flat directions can also be parameterized by the vev's of the gauge invariant operators $M_{i j}=Q_{i} \bar{Q}_{j}$. These composite degrees of freedom give a better (weakly coupled) description near the origin of moduli space where the theory is in a confined regime.

In this model a unique nonperturbative superpotential is allowed by the symmetries (Affleck-Dine-Seiberg, 1984a) 


$$
W_{d y n}=\left(\frac{\Lambda^{3 N_{c}-N_{f}}}{\operatorname{det}(Q \bar{Q})}\right)^{\frac{1}{N_{c}-N_{f}}}
$$

where $\Lambda$ is the renormalization group invariant scale of the theory. It was shown (AffleckDine-Seiberg, 1984a; Cordes, 1986) that this superpotential is in fact generated by instanton effects for $N_{f}=N_{c}-1$. It is generated by gaugino condensation in all other cases.

Before proving this last statement, let us pause for a moment and discuss the relation between the renormalization group invariant scales of the microscopic and effective theories. Suppose the microscopic theory is $S U\left(N_{c}\right)$ with $N_{f}$ flavors. As has been mentioned above the Wilsonian coupling (Shifman and Vainshtein, 1986; Shifman and Vainshtein, 1991) of the theory runs only at one loop

$$
\frac{1}{g^{2}(\mu)}=\frac{1}{g^{2}(M)}+\frac{b_{0}}{16 \pi^{2}} \ln \left(\frac{\mu}{M}\right) .
$$

Suppose also that at a scale $v$ some fields in the theory become massive, and the physics below this scale is described by an $S U\left(N_{c}^{\prime}\right)$ gauge group with $N_{f}^{\prime}$ flavors. The Wilsonian coupling of the effective theory is

$$
\frac{1}{g_{L}^{2}(\mu)}=\frac{1}{g_{L}^{2}(M)}+\frac{\tilde{b}_{0}}{16 \pi^{2}} \ln \left(\frac{\mu}{M}\right) .
$$

In equations (A16) and (A17) $b_{0}=3 N_{c}-N_{f}$ and $\tilde{b}_{0}=3 N_{c}^{\prime}-N_{f}^{\prime}$ are the $\beta$-function coefficients. But the couplings should be equal at the scale $\mu=v$. This allows us to derive scale matching conditions. For example, take an $S U\left(N_{c}\right)$ theory with $N_{f}$ flavors. Its renormalization group invariant scale is given by

$$
\Lambda^{3 N_{c}-N_{f}}=\mu^{3 N_{c}-N_{f}} \exp \left(-\frac{8 \pi^{2}}{g^{2}}\right) .
$$

If one of the matter fields is massive with mass $m \gg \Lambda$, the effective theory has $N_{f}-1$ flavors and its scale is

$$
\Lambda_{L}^{3 N_{c}-N_{f}+1}=\mu^{3 N_{c}-N_{f}+1} \exp \left(-\frac{8 \pi^{2}}{g^{2}}\right) .
$$

Requiring equality of couplings at the scale of the mass we find 


$$
\Lambda_{L}^{3 N_{c}-N_{f}+1}=m \Lambda^{3 N_{c}-N_{f}}
$$

In a general case the equation above becomes

$$
\Lambda_{L}^{\tilde{b}_{0}}=v^{\tilde{b}_{0}-b_{0}} \Lambda^{b_{0}}
$$

where $v$ represents a generic vev and/or mass in the theory.

Now, in the $N_{f}=N_{c}-1$ theory with small masses $m \ll \Lambda$ an instanton calculation (Affleck-Dine-Seiberg, 1984a; Cordes, 1986) is reliable and gives (A15). Due to the holomorphicity of the superpotential the result can be extrapolated into the region of moduli space where one flavor, say $N_{f}$ 'th, is heavy, $m_{N_{f} N_{f}} \gg \Lambda$. It decouples from the low energy effective theory. Solving the equations of motion for the heavy field and using the scale matching condition (A21) one finds the superpotential (A15) for the effective theory with $N_{f}=N_{c}-2$ flavors. In the low energy effective theory this superpotential can be interpreted as arising from gaugino condensation. One can continue this procedure by induction and not only derive the superpotential for arbitrary $N_{f}<N_{c}$ but also fix the numerical coefficient in front of the superpotential. (This coefficient can be absorbed into the definition of $\Lambda$, and we shall set it to 1 most of the time.)

Even though the classical flat directions are lifted in the massless theory by the superpotential (A15), the scalar potential

$$
V=\sum_{i}\left(\left|\frac{\partial W}{\partial Q_{i}}\right|^{2}+\left|\frac{\partial W}{\partial \bar{Q}}\right|^{2}\right)
$$

tends to zero as $Q=\bar{Q} \rightarrow \infty$ and as a result theory does not possess a stable vacuum state. In our discussion of DSB we discuss examples where flat directions may be not only lifted but stabilized due to nonperturbative effects (see Section VIB).

One could lift classical flat directions by adding a mass term to the superpotential

$$
W_{\text {tree }}=m_{i j} Q_{i} \bar{Q}_{j}
$$

Note that this superpotential explicitly breaks the $U(1)_{R}$ symmetry. In Section [IIB we argue that this is often a signal of unbroken supersymmetry. It is easy to find the supersymmetric vacua in this model. In terms of the meson fields they are given by 


$$
M_{i j}=\left(\operatorname{det}(m) \Lambda^{3 N_{c}-N_{f}}\right)^{1 / N_{c}}\left(\frac{1}{m_{i j}}\right) .
$$

It is also worth noting that if some number of matter fields are massive they decouple from the low energy theory and can be integrated out. Solving the equations of motion for the massive fields one can find the superpotential of Eq. (A15) and the solution (A24) for the vev's of the remaining light fields.

\section{5. $N_{f}=N_{c}$ : Quantum Moduli Space}

Additional flat directions exist for $N_{f}=N_{c}$. The most general expression for the flat directions is

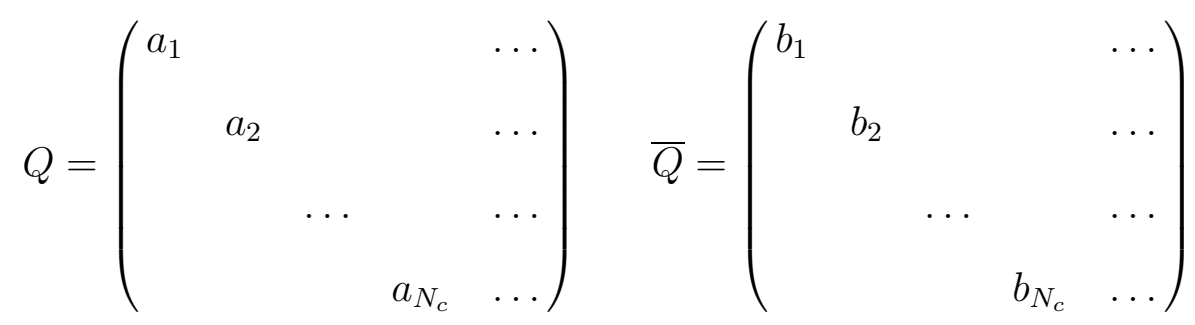

subject to the condition

$$
\left|a_{i}\right|^{2}-\left|b_{i}\right|^{2}=v^{2}
$$

As was mentioned above, the flat directions can be parameterized by vevs of gauge invariant polynomials. In this case new flat directions can be represented by fields with the quantum numbers of baryons $33=Q^{N}$ and antibaryons $\bar{B}=\bar{Q}^{N}$. Note, however, that due to the Bose statistics of the superfields, the gauge invariant polynomials obey the constraint, classically,

$$
\operatorname{det} M-B \bar{B}=0 \text {. }
$$

Seiberg (1994; 1995) ) showed that this constraint is modified quantum mechanically,

\footnotetext{
${ }^{33}$ Summation over both color and flavor indices is implied.
} 


$$
\operatorname{det}(M)-B \bar{B}=\Lambda^{2 N} .
$$

We refer the reader to (Seiberg, 1994; [ntriligator and Seiberg, 1996; Peskin, 1997; Shifman, 1997) for a detailed explanation of this result.

It is often convenient to enforce this quantum mechanical constraint by introducing a Lagrange multiplier term in the superpotential

$$
W=A\left(\operatorname{det}(M)-B \bar{B}-\Lambda^{2 N}\right)+m_{i j} M_{i j} .
$$

Once again the validity of this superpotential can be verified in the limit that some of the matter fields are heavy and decouple from the low energy theory. Integrating them out leads to the superpotential (A15) for the light matter.

Naively, the Kähler potential of the $N_{f}=N_{c}$ theory is singular at the origin. This corresponds to the fact that at the origin, the full gauge group $S U\left(N_{c}\right)$ is restored and additional degrees of freedom become massless. This singular point, however, does not belong to the quantum moduli space. $S U\left(N_{c}\right)$ cannot be restored because of the constraint (A28) and the Kähler potential in terms of composite degrees of freedom is non-singular. In the infrared mesons and baryons represent a good description of the theory. One of many non-trivial tests they pass is 't Hooft anomaly matching conditions (t Hooft, 1980). Far from the origin the quantum moduli space is very close to classical one and the elementary degrees of freedom should represent a good (weakly coupled) description of the theory.

\section{6. $N_{f}=N_{c}+1$}

In this case there are $N_{f}$ baryons and antibaryons transforming under the global $S U\left(N_{f}\right)_{L} \times S U\left(N_{f}\right)_{R}$ as $\left(N_{f}, 1\right)$ and $\left(1, \bar{N}_{f}\right)$ respectively. Classically, the gauge invariants obey the constraints

$$
\begin{aligned}
& \operatorname{det}(M)-B_{i} M_{i j} \bar{B}_{j}=0, \\
& B_{i} M_{i j}=M_{i j} \bar{B}_{j}=0 .
\end{aligned}
$$


These constraints are not modified quantum mechanically. One can easily see this by adding a tree level superpotential $W_{\text {tree }}=\sum_{i j} m_{i j} M_{i j}$. Holomorphy guarantees that meson vevs are given by Eq. (A24). Taking various limits of the mass matrix one can see that the mesons $M_{i j}$ can have any values on the moduli space. This can also be shown for the baryons.

In terms of the elementary fields the Kähler potential is singular at the origin reflecting the fact that $S U\left(N_{c}\right)$ is restored there and additional degrees of freedom become massless. In terms of composite degrees of freedom the Kähler potential is regular, and they represent a suitable infrared description of the theory. As in the case $N_{f}=N_{c}$, 't Hooft anomaly matching conditions are satisfied by the effective description. In this model, the constraints can be implemented by the superpotential

$$
W=\frac{1}{\Lambda^{2 N_{c}-1}}\left(B_{i} M_{i j} \bar{B}_{j}-\operatorname{det} M\right) .
$$

Adding mass for one flavor correctly leads to the $N_{f}=N_{c}$ model.

\section{7. $N_{f}>N_{c}+1$ : Dual Descriptions of the Infrared Physics}

We shall start from the case $\frac{3}{2} N_{c}<N_{f}<3 N_{c}$. This theory flows to an infrared fixed point (Seiberg, 1995). Seiberg (1995) suggested that in the vicinity of the infrared fixed point the theory admits a dual, "magnetic", description with the same global symmetries but in terms of a theory with a different gauge group. This theory is based on the gauge group $S U\left(N_{f}-N_{c}\right)$ with $N_{f}$ flavors of $q$ and $\bar{q}$ transforming as fundamentals and antifundamentals respectively, as well as gauge-singlet fields $M$, corresponding to the mesons of the original ("electric") theory. The global-symmetry charges are given by

$$
\begin{aligned}
& S U\left(N_{f}\right)_{L} \times S U\left(N_{f}\right)_{R} \times U(1)_{B} \times U(1)_{R} \\
& \begin{array}{lllll}
\bar{N}_{f} & 1 & \frac{N_{c}}{N_{f}-N_{c}} & \frac{N_{c}}{N_{f}}
\end{array} \\
& \bar{q} \quad 1 \quad N_{f} \quad-\frac{N_{c}}{N_{f}-N_{c}} \quad \frac{N_{c}}{N_{f}} \\
& \begin{array}{lllll}
M & N_{f} & \bar{N}_{f} & 0 & 2 \frac{N_{f}-N_{c}}{N_{c}}
\end{array}
\end{aligned}
$$

The magnetic theory also flows to a fixed point. However, in the magnetic theory a tree level superpotential is allowed by symmetries 


$$
W=M q \bar{q} .
$$

In the presence of this superpotential the theory flows to a new fixed point which is identical to the fixed point of the "electric" theory.

At the fixed point the superconformal symmetry can be used to understand the behavior of the theory. For example the scaling dimensions of the gauge invariant operators are known. The exact beta function for the coupling in 1PI action (in the electric description) is given by (Novikov et ad, 1983; Shifman and Vainshtein, 1986; Shifman and Vainshtein, 1991)

$$
\begin{aligned}
& \beta(g)=\frac{g^{2}}{16 \pi^{2}} \frac{3 N_{c}-N_{f}+N_{f} \gamma\left(g^{2}\right)}{1-N_{c} \frac{g^{2}}{8 \pi^{2}}} \\
& \gamma(g)=-\frac{g^{2}}{16 \pi^{2}} \frac{N_{c}^{2}-1}{N_{c}}+\mathcal{O}\left(g^{4}\right) .
\end{aligned}
$$

At the zero of the $\beta$-function the anomalous dimension is $\gamma=-3 N_{c} / N_{f}+1$, and one finds

$$
D(Q \bar{Q})=2+\gamma=3 \frac{N_{f}-N_{c}}{N_{f}} .
$$

The dimension of the baryon operators can be determined by exploiting the $\mathrm{R}$ symmetry (Seiberg, 1995)

$$
D(B)=D(\bar{B})=\frac{3 N_{c}\left(N_{f}-N_{c}\right)}{2 N_{f}} .
$$

This allows one to determine the scaling of the Kähler potential near the fixed point both in the electric and in the magnetic description

$$
\begin{aligned}
& K_{e} \sim(Q \bar{Q})^{\frac{2 N_{f}}{3\left(N_{f}-N_{c}\right)}}, \\
& K_{m} \sim(q \bar{q})^{\frac{2 N_{f}}{3 N_{c}}} .
\end{aligned}
$$

Let us summarize the correspondence between the electric and magnetic theories:

$$
\begin{aligned}
M_{i j}=Q_{i} \bar{Q}_{j} & \rightarrow M_{i j}, \\
W=m_{i j} M_{i j} & \rightarrow W=m_{i j} M_{i j}+M_{i j} q_{i} \bar{q}_{j}, \\
b, \bar{b} & \rightarrow B, \bar{B} .
\end{aligned}
$$


By performing a second duality transformation one can verify that in fact the magnetic meson is identified with the composite electric meson through the equations of motion.

The scales of the electric and the magnetic theories are related by

$$
\Lambda^{3 N_{c}-N_{f}} \tilde{\Lambda}^{3\left(N_{f}-N_{c}\right)-N_{f}}=(-1)^{N_{f}-N_{c}} \mu^{N_{f}},
$$

where the scale $\mu$ is needed to map the composite electric meson $Q \bar{Q}$ into an elementary magnetic meson $M$. These fields have the same dimension at the infrared fixed point, but different dimensions in the ultraviolet.

If the number of flavors is $N_{c}+1<N_{f}<\frac{3}{2} N_{c}$ one could construct a dual description in a similar way. In that case only the electric description is asymptotically free and makes sense in the ultraviolet.

\section{Other Models}

There are numerous generalizations of the results presented in previous subsections to theories with different gauge groups and matter fields. Here we mention some of the generalizations which will be useful for our discussion of supersymmetry breaking.

Results analogous to those for SQCD can be found for $S P(N)$ theories with $N_{f}$ flavors of matter fields transforming in the fundamental representation [Intriligator and Pouliot, 1995). The one loop $\beta$-function coefficient is given by

$$
b_{0}=3\left(N_{c}+1\right)-N_{f} .
$$

This can be compared to the one loop $\beta$-function of the $S U(N)$ models given in Eq. (A16) In fact, one can find many of the results for $S P(N)$ theories by making the substitution $N_{c} \rightarrow N_{c}+1$ in the expressions for $S U(N)$ models (and rewriting determinants as Pfaffians). The theory does not have baryons for any number of flavors. The supersymmetric vacuum is given by

\footnotetext{
${ }^{34}$ In our notation $S P(1)=S U(2)$, and $N_{f}$ flavors correspond to $2 N_{f}$ fields.
} 


$$
M_{i j}=Q_{i} Q_{j}=\left(\operatorname{Pf}(m) \Lambda^{3\left(N_{c}+1\right)}\right)^{\frac{1}{N_{c}+1}}\left(\frac{1}{m_{i j}}\right) .
$$

We can easily see that for $N_{f}=N_{c}+1$, the quantum constraint is different from the classical one,

$$
P f(M)=\Lambda^{2\left(N_{c}+1\right)} .
$$

If the number of flavors is $N_{f}>N_{c}+2$ there is a dual description analogous to the one for the $S U(N)$ theories.

We conclude this section by mentioning several other classes of models which are useful in the study of the supersymmetry breaking. Poppitz and Trivedi (1996) studied quantum moduli space and exact superpotentials in $S U(N)$ gauge theories with matter in the antisymmetric tensor, fundamental, and antifundamental representations. In the case without fundamental fields Pouliot (1996) has constructed a dual by using Berkooz's (1996) deconfining trick. In (Poppitz-Shadmi-Trived, 1996a) duality was studied in the product group theories and it was shown that dual models can be constructed by using single group duality. 


\section{REFERENCES}

Affleck, I., M. Dine, and N. Seiberg, 1984a, "Dynamical Supersymmetry Breaking in Supersymmetric QCD", Nucl. Phys. B 241, 493-534.

Affleck, I., M. Dine, and N. Seiberg, 1984b, "Dynamical Supersymmetry Breaking in Chiral Theories", Phys. Lett. B 137, 187-192.

Affleck, I., M. Dine, and N. Seiberg, 1984c, "Exponential Hierarchy from Dynamical Supersymmetry Breaking", Phys. Lett. B 140, 59-62.

Affleck, I., M. Dine, and N. Seiberg, 1984d, "Calculable Nonperturbative Supersymmetry Breaking", Phys. Rev. Lett. 52, 1677-1680.

Affleck, I., M. Dine, and N. Seiberg, 1985, "Dynamical Supersymmetry Breaking in Four Dimensions and its Phenomenological Implications", Nucl. Phys. B 256, 557-599.

Amati, D., G. Rossi, and G.Veneziano, 1985, "Instanton Effects in Supersymmetric Gauge Theories", Nucl. Phys. B 249, 1-41.

Arkani-Hamed, N., M. Dine and S.P. Martin, 1998, "Dynamical Supersymmetry Breaking in Models with a Green-Schwarz Mechanism", Phys. Lett. B 431, 329-338; eprint hepph/9803432.

Arkani-Hamed, N., and J. March-Russell, and H. Murayama, 1998 "Building Models Of Gauge Mediated Supersymmetry Breaking Without A Messenger Sector", Nucl. Phys. B 509, 3-32; eprint hep-ph/9701286.

Arkani-Hamed, N., and H. Murayama, 1998, "Renormalization Group Invariance of Exact Results in Supersymmetric Gauge Theories", Phys. Rev. D 57, 6638-6648; eprint hepth/9705189.

Bagger, J., E. Poppitz, and L. Randall, 1994, "The R-axion from Dynamical Supersymmetry Breaking", Nucl. Phys. B 426, 3-18; eprint hep-ph/9405345. 
Bagger, J., and J. Wess, 1991, Supersymmetry and Supergravity, 2nd edition, (Princeton University Press, Princeton).

Bagger, J., and E. Witten, 1982, "Quantization of Newton's Constant in Certain Supergravity Theories", Phys. Lett. B 115, 202-206.

Berkooz, M., 1996, "The Dual of Supersymmetric $S U(2 K)$ with an Antisymmetric Tensor and Composite Dualities", Nucl. Phys. B 466, 75-84; eprint hep-th/9512024.

Binetruy, P., and E. Dudas, 1996, "Gaugino Condensation and the Anomalous U(1)", Phys. Lett. B 389, 503-509; eprint hep-th/9607172.

de Boer, J., K. Hori, H. Ooguri, and Y. Oz, 1998, "Branes and Dynamical Supersymmetry Breaking", Nucl. Phys. B 522, 20-68; eprint hep-th/9801060.

Brodie, J., P. Cho, and K. Intriligator, 1998, "Misleading Anomaly Matchings?", Phys. Lett. B 429, 319-326; eprint hep-th/9802092.

Chacko, Z., M.A. Luty and E. Ponton, 1998 "Calculable Dynamical Supersymmetry Breaking on Deformed Moduli Spaces", J. High Ener. Phys. 12, 016; eprint hep-th/9810253.

Cheng, C.-H., and Y. Shadmi, 1998, "Duality in the Presence of Supersymmetry Breaking", Nucl. Phys. B 531, 125-150; eprint hep-th/9801146.

Chou, C.-L., 1997, "Models of Dynamical Supersymmetry Breaking from a $S U(2 K+3)$ Gauge Model", Phys. Lett. B 391, 329-334; eprint hep-th/9605119.

Clark, T. E., O. Piguet, K. Sibold, 1979, "The Absence of Radiative Corrections to the Axial Current Anomaly in Supersymmetric QED”, Nucl. Phys. B 159, 1-15.

Cordes, S., 1986, "The Instanton-Induced Superpotential in Supersymmetric QCD", Nucl. Phys. B 273, 629-648.

Csaki, C., R. Leigh, L. Randall, and W. Skiba, 1996, "Supersymmetry Breaking through Confining and Dual Theory Gauge Dynamics", Phys. Lett. B 387, 791-795; eprint hep- 
th/9607021.

Csaki, C., L. Randall, and W. Skiba, 1996, "More Dynamical Supersymmetry Breaking", Nucl. Phys. B 479, 65-81; eprint hep-th/9605108.

Csaki, C., M. Schmaltz, and W. Skiba, 1997a, "Exact Results and Duality for $S P(2 N)$ SUSY Gauge Theories with an Antisymmetric Tensor", Nucl. Phys. B 487, 128-140; eprint hep-th/9607210.

Csaki, C., M. Schmaltz, and W. Skiba, 1997b, "Confinement in $N=1$ SUSY Gauge Theories and Model Building Tools", Phys. Rev. D 55, 7840-7858; eprint hep-th/9612207.

Csaki, C., M. Schmaltz, and W. Skiba, 1997c, "A Systematic Approach to Confinement in N=1 Supersymmetric Gauge Theories", Phys. Rev. Lett. 78, 799-802; eprint hepth/9610139.

Dimopoulos, S., M. Dine, S. Raby, and S. Thomas, 1996, "Experimental Signatures of LowEnergy Gauge Mediated Supersymmetry Breaking", Phys. Rev. Lett. 76, 3494-3497; eprint hep-ph/9601367.

Dimopoulos, S., G. Dvali, R. Rattazzi, and G. Giudice, 1998, "Dynamical Soft Terms with Unbroken Supersymmetry", Nucl. Phys. B 510, 12-38; eprint hep-ph/9705307.

Dimopoulos, S., and H. Georgi, 1981, "Softly Broken Supersymmetry And SU(5)", Nucl. Phys. B 193, 150-162.

Dine, M., A. Nelson, Y. Nir, and Y. Shirman, 1996, "New Tools for Low-Energy Dynamical Supersymmetry Breaking", Phys. Rev. D 53, 2658-2669; eprint hep-ph/9507378.

Dine, M, A. Nelson, and Y. Shirman, 1995, "Dynamical Supersymmetry Breaking Simplified", Phys. Rev. D 51, 1362-1370; eprint hep-ph/9408384.

Dvali, G., and Z. Kakushadze, 1998, "Dynamical Flavor Hierarchy and Heavy Top", Phys. Lett. B 426, 78-81; eprint hep-ph/9707287. 
Dvali, D., and A. Pomarol, 1996, "Anomalous U(1) as a Mediator of Supersymmetry Breaking", Phys. Rev. Lett. 77, 3728-3731; eprint hep-ph/9607383.

Einhorn, M. B., and D. T. R. Jones, 1983, "Absence of Goldstino Decoupling in Hierarchical Superunified Models", Phys. Lett. B 128, 174-178.

Fayet, P., 1975, "Spontaneous Supersymmetry Breaking without Gauge Invariance", Phys. Lett. B 58, 67-70.

Fayet, P., and J. Iliopoulos, 1974, "Spontaneously Broken Supergauge Symmetries and Goldstone Spinors", Phys. Lett. B 51, 461-464.

Ferrara, S., L. Girardello, F. Palumbo, 1979, "A General Mass Formula in Broken Supersymmetry", Phys. Rev. D 20, 403-408.

Ferrara, S., J. Iliopoulos and B. Zumino, 1974, "Supergauge Invariance And The Gell-MannLow Eigenvalue", Nucl. Phys. B 77, 413-419.

Fischler, W., H. P. Nilles, J. Polchinski, S. Raby, and L. Susskind, 1981, "Vanishing Renormalization of the D-term in Supersymmetric $U(1)$ Theories", Phys. Rev. Lett. 47, 757-759.

Giudice, G. F., and R. Rattazzi, 1998, "Theories with Gauge Mediated Supersymmetry Breaking"; eprint hep-ph/9801271.

Green, M., and J. Schwarz, 1984, "Anomaly Cancellations In Supersymmetric D = 10 Gauge Theory Require SO(32)", Phys. Lett. B 149, 117-122.

Grisaru, M. T., M. Rocek, and W. Siegel, 1979, "Improved Methods For Supergraphs", Nucl. Phys. B 159, 429-450.

t'Hooft, G., 1980, in Recent Developments in Gauge Theories, edited by G. t' Hooft et al., (Plenum Press, New York, USA).

Huq, M., 1976, "Spontaneous Breakdown of fermion number conservation and supersymmetry", Phys. Rev. D 14, 3548-3556. 
Intriligator, K., and P. Pouliot, 1995, "Exact Superpotentials, Quantum Vacua and Duality in $S P\left(N_{c}\right)$ Gauge Theories", Phys. Lett. B 353, 471-476; eprint hep-th/9505006.

Intriligator, K., and N.Seiberg, 1994, "Phases of N=1 Supersymmetric Gauge Theories in Four Dimensions", Nucl. Phys. B 431, 551-565; eprint hep-th/9408155.

Intriligator, K., and N. Seiberg, 1996, "Lectures on Supersymmetric Gauge Theories and Electric-Magnetic Duality", Nucl. Phys. Proc. Suppl. BC 45, 1-28; eprint hep-th/9509066.

Intriligator, K., N. Seiberg, and S. Shenker, 1995, "Proposal for a Simple Model of Dynamical SUSY Breaking", Phys. Lett. B 342, 152-154; eprint hep-th/9410203.

Intriligator, K., and S. Thomas, 1996a, "Dynamical Supersymmetry Breaking on Quantum Moduli Spaces", Nucl. Phys. B 473, 121-140; eprint hep-th/9603158.

Intriligator, K., and S. Thomas, 1996b, "Dual Descriptions of Supersymmetry Breaking", eprint hep-th/9608046.

Izawa, K.-I., and T. Yanagida, 1996, "Dynamical Supersymmetry Breaking in Vector-like Gauge Theories", Prog. Theor. Phys. 95, 829-830; eprint hep-th/9602180.

Konishi, K., 1984, "Anomalous Supersymmetry Transformation of some Composite Operators in SQCD", Phys. Lett. B 135, 439-449.

Kovner, K., and M. Shifman, 1997, "Chirally Symmetric Phase of Supersymmetric Gluodynamics", Phys. Rev. D 56, 2396-2402; eprint hep-th/9702174.

Leigh, R., L. Randall, and R. Rattazzi, 1997, "Unity of Supersymmetry Breaking Models", Nucl. Phys. B 501, 375-408; eprint hep-ph/9704246.

Luty, M., and W. Taylor, 1996, "Varieties of Vacua in Classical Supersymmetric Gauge Theories", Phys. Rev. D 53, 3399-3405; eprint hep-th/9506098.

Luty, M., and J. Terning, 1998, "New Mechanisms of Dynamical Supersymmetry Breaking and Direct Gauge Mediation", Phys. Rev. D 57, 6799-6806; eprint hep-ph/9709306. 
Lykken, J., E. Poppitz, S. Trivedi, 1999, "Branes with GUTs and Supersymmetry Breaking", Nucl. Phys. B 543, 105-121; eprint hep-th/9806080.

Meurice, Y., and G. Veneziano, 1984, "SUSY Vacua versus Chiral Fermions", Phys. Lett. B 141, 69-72.

Murayama, H., 1995, "Studying Non-calculable Models of Dynamical Supersymmetry Breaking", Phys. Lett. B 355, 187-192; eprint hep-th/9505082.

Murayama, H., 1997, "A Model of Direct Gauge Mediation”, Phys. Rev. Lett. 79, 18-21; eprint hep-ph/9705271.

Nelson, A., 1998, "Dynamical Supersymmetry Breaking”, Nucl. Phys. Proc. Suppl. 62, 261265; eprint hep-ph/9707442.

Nelson, A., and N. Seiberg, 1994, "R-symmetry Breaking versus Supersymmetry Breaking", Nucl. Phys. B 416, 46-62; eprint hep-ph/9309299.

Nelson, A,. and S. Thomas, 1996, unpublished.

Nilles, H. P., 1984, "Supersymmetry, Supergravity And Particle Physics", Phys. Rep. 110, $1-159$.

Novikov, V., M. Shifman, A. Vainshtein, and V. Zakharov, 1983, "Exact Gell-Mann-Low Function of Supersymmetric Theories from Instanton Calculus", Nucl. Phys. B 229, 381393.

O’Raifeartaigh, L., 1975, "Spontaneous Symmetry Breaking for Chiral Scalar Superfields", Nucl. Phys. B 96, 331-352.

Peskin, M., 1997, "Duality in Supersymmetric Yang Mills Theory", in Fields, Strings and Duality, Eds. C. Efthimiou and B. Greene, (World Scientific); eprint hep-th/9702094.

Polchinski, J., 1983, "Effective Potentials for Supersymmetric Three-scale Hierarchies", Phys. Rev. D 27, 1320-1330. 
Poppitz, E., 1998, "Dynamical Supersymmetry Breaking: Why and How", Int. J. Mod. Phys. A 13, 3051-3080; eprint hep-ph/9710274.

Poppitz, E., and L. Randall, 1996, "Low-Energy Kähler Potentials in Supersymmetric Gauge Theories with (Almost) Flat Directions", Phys. Lett. B 336, 402-408; eprint hepth/9407185.

Poppitz, E., Y. Shadmi, and S. Trivedi, 1996a, "Duality and Exact Results in Product Group Theories", Nucl. Phys. B 480, 125-169; eprint hep-th/9605113.

Poppitz, E., Y. Shadmi, and S. Trivedi, 1996b, "Supersymmetry Breaking and Duality in $S U(N) \times S U(N-M)$ Gauge Theories", Phys. Lett. B 388, 561-568; eprint hep-th/9606184.

Poppitz, E., and S. Trivedi, 1996, "Some Examples of Chiral Moduli Spaces and Dynamical Supersymmetry Breaking", Phys. Lett. B 365, 125-131; eprint hep-th/9507169.

Poppitz, E., and S. Trivedi, 1997, "New Models of Gauge and Gravity Mediated Supersymmetry Breaking", Phys. Rev. D 55, 5508-5519; eprint hep-ph/9609529.

Poppitz, E., and S.P. Trivedi, 1998, "Dynamical Supersymmetry Breaking"; eprint hepth/9803107.

Pouliot, P., 1996, "Duality in SUSY $S U(N)$ with an Antisymmetric Tensor", Phys. Lett. B 367, 151-156; eprint hep-th/9510148.

Pouliot, P., and M. Strassler, 1996, "Duality and Dynamical Supersymmetry Breaking in $\operatorname{Spin}(10)$ with a Spinor", Phys. Lett. B 375, 175-180; eprint hep-th/9602031.

Salam, A., and J. Strathdee, 1974, "On Goldstone Fermions", Phys. Lett. B 49, 465.

Seiberg, N., 1993, "Naturalness Versus Supersymmetric Non-renormalization Theorems", Phys. Lett. B 318, 469-475; eprint hep-th/9309335.

Seiberg, N., 1994, "Exact Results on the Space of Vacua of Four-Dimensional SUSY Gauge Theories", Phys. Rev. D 49, 6857-6863; eprint hep-th/9402044. 
Seiberg, N., 1995, "Electric-Magnetic Duality in Supersymmetric Nonabelian Gauge Theories", Nucl. Phys. B 435, 129-146; eprint hep-th/9411149.

Seiberg, N., and E. Witten, 1994a, "Electric-Magnetic Duality, Monopole Condensation, And Confinement In $N=2$ Supersymmetric Yang-Mills Theory", Nucl. Phys. B 426, 19-52; 430, 485-486; eprint hep-th/9407087.

Seiberg, N., and E. Witten, 1994b, "Monopoles, Duality and Chiral Symmetry Breaking in N=2 Supersymmetric QCD", Nucl. Phys. B 431, 484-550; eprint hep-th/9408099.

Shadmi, Y., 1997, "Gauge Mediated Supersymmetry Breaking without Fundamental Singlets", Phys. Lett. B 405, 99-107; eprint hep-ph/9703312.

Shifman, M., 1997, "Nonperturbative Dynamics in Supersymmetric Theories", Prog. Part. Nucl. Phys. 39, 1-116; eprint hep-th/9704114.

Shifman, M., and A. Vainshtein, 1986, "Solution of the Anomaly Puzzle in SUSY Gauge Theories and the Wilson Operator Expansion", Nucl. Phys. B 277, 456-486.

Shifman, M., and A. Vainshtein, 1988, "On Gluino Condensation in Supersymmetric Gauge Theories. $S U(N)$ and $O(N)$ groups", Nucl. Phys. B 296, 445-461.

Shifman, M., and A. Vainshtein, 1991, "On Holomorphic Dependence and Infrared Effects in Supersymmetric Gauge Theories", Nucl. Phys. B 359, 571-580.

Shifman, M., and A. Vainshtein, 1999, "Instantons versus Supersymmetry: Fifteen Years Later"; eprint hep-th/9902018.

Shirman, Y., 1996, "Dynamical Supersymmetry Breaking versus Run-away Behavior in Supersymmetric Gauge Theories", Phys. Lett. B 389, 287-293; eprint hep-th/9608147.

Skiba, W., 1997, "Dynamical Supersymmetry Breaking", Mod. Phys. Lett. A 12, 737-750; eprint hep-th/9703159.

Stump, D. R., M. Wiest and C.P. Yuan, 1996, "Detecting a Light Gravitino at Linear Collider 
to Probe the SUSY Breaking Scale", Phys. Rev. D 54, 1936-1943; eprint hep-ph/9601362.

Thomas, S., 1998, "Recent Developments in Dynamical Supersymmetry Breaking"; eprint hep-th/9801007.

ter Veldhuis, T., 1996, "The Mass Spectrum In a Model with Calculable Dynamical Supersymmetry Breaking", Phys. Lett. B 367, 157-162; eprint hep-th/9510121.

ter Veldhuis, T., 1998, "Low Energy Behavior of Some Models with Dynamical Supersymmetry Breaking", Phys. Rev. D 58, 015010; eprint hep-th/9802125.

Wess, J., and B. Zumino, 1974, "A Lagrangian Model Invariant Under Supergauge Transformations", Phys. Lett. B 49, 52-54.

Witten, E., 1981a, "Dynamical Breaking of Supersymmetry", Nucl. Phys. B 188, 513-554.

Witten, E., 1981b, "Mass Hierarchies in Supersymmetric Theories", Phys. Lett. B 105, 267-271.

Witten, E., 1982 "Constraints on Supersymmetry Breaking", Nucl. Phys. B 202, 253-316.

Witten, E., 1998, "Toroidal Compactifications without Vector Structure", J. High Ener. Phys. 02, 006; eprint hep-th/9712028.

Zumino, B., 1981, "Spontaneous Breaking of Supersymmetry", preprint LBL-13691, Heisenberg Simp., 137. 